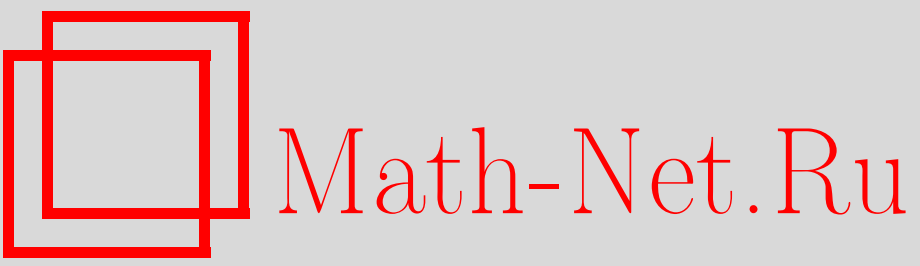

Д. А. Попов, Восстановление характеристических функций в двумерной радоновской томографии, УМН, 1998, том 53, выпуск 1, 115-198

DOI: https://doi.org/10.4213/rm1

Использование Общероссийского математического портала Math-Net.Ru подразумевает, что вы прочитали и согласны с пользовательским соглашением

http://www.mathnet.ru/rus/agreement

Параметры загрузки:

IP: 54.198 .187 .58

26 апреля 2023 г., 13:14:13 


\title{
ВОССТАНОВЛЕНИЕ ХАРАКТЕРИСТИЧЕСКИХ ФУНКЦИЙ В ДВУМЕРНОЙ РАДОНОВСКОЙ ТОМОГРАФИИ
}

\author{
Д. А. Попов \\ СОДЕРЖАНИЕ
}

$\S 1$. Введение. Математическая модель томографа .................... 116

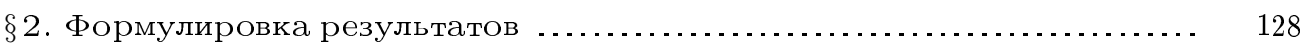

$\S 3$. Ошибки восстановления и ряды осциллирующих интегралов ....... 134

$\S 4$. Анализ критических точек .................................... 144

$\S 5$. Оценка ошибки дискретизации ................................ 151

5.1. Предварительные замечания ............................... 151

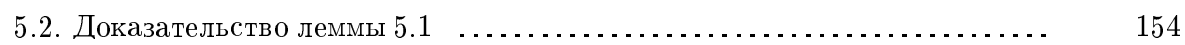

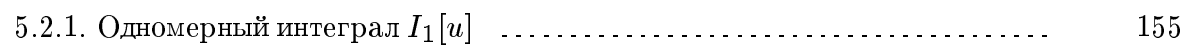

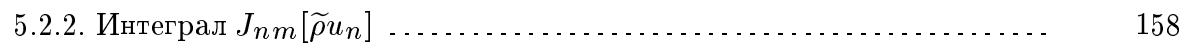

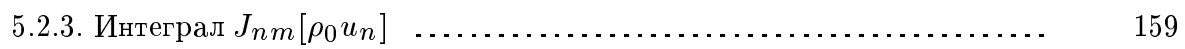

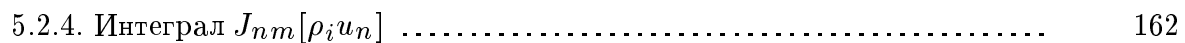

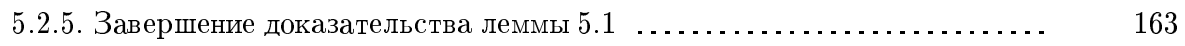

5.3. Доказательство пп. 1, 2 теоремы 2.1 ........................... 166

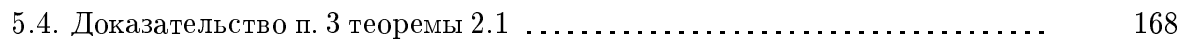

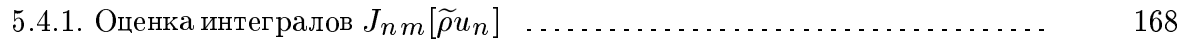

5.4.2. Оценка интеграла $J_{n m}\left[\rho_{0} u_{n}\right]$ и завершение доказательства теоре- 170

5.5. Формальньй показатель сходимости и точность полученных оценок $\quad . . .177$

5.6. Заключительные замечания ....................................... 180

Приложение. Оценки осциллирующих интегралов ..................... 181

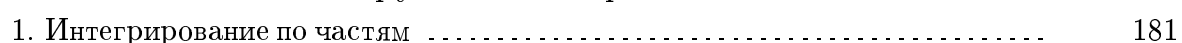

2. Остаточньй член в методе стационарной фазы ...................... 182

3. Равномерные оценки одномерных ОИ .............................. 184

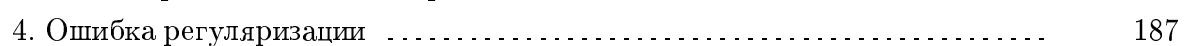

5. Специальньй класс двумерных ОИ

6. Оценки главных значений ОИ ................................ 194

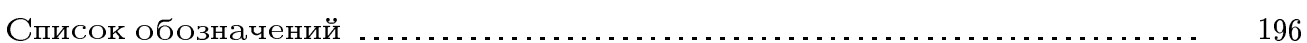

Список литературы ................................................... 197

(C) Д.А. Попов 1998 


\section{§1. Введение. Математическая модель томографа}

В настоящее время под томографией понимается широкий круг методов, позволяющих восстановить функцию, характеризующую внутреннюю структуру объекта, по известным интегралам от этой функции [1]-[4]. Данная работа посвящена только задаче двумерной радоновской томографии. Заметим, что именно решение этой задачи нашло свое практическое воплошение в создании действуюших рентгеновских томографов. Точное значение термина "радоновская томография” будет объяснено ниже.

Автор искренне благодарен В. П. Паламодову за полезные обсуждения и критику.

Математические вопросы томографии изложены в ряде работ, среди которых отметим [5]-[11]. Строгая формулировка задачи восстановления в томографии эквивалентна выбору некоторой математической модели томографа (ММТ), которая должна включать описание

1) класса восстанавливаемых функиий (обвектов) - $\{\mu\}$,

2) массива исходных данных, т.е. набора функиионалов от $\mu \in\{\mu\}$, по которому производится восстановление,

3) алгоритма восстановления.

При описании ММТ будем следовать работе [12], где подробно изложены различные вопросы построения ММТ.

Объектом восстановления в двумерной томографии является функция $\mu$ на плоскости $\mathbb{R}^{2}$ с выделенным началом координат $\vec{O}$ - центром сканирования. Обозначая восстанавливаемую функцию через $\mu$, мы отдаем дань традиции - в рентгеновской томографии восстанавливается коэффициент поглошения рентгеновских лучей, обозначаемьй через $\mu$.

Класс $\{\mu\}$ восстанавливаемых функиий - это, по определению, функщии с компактньм носителем в круге единичного диаметра, которые можно представить в виде

$$
\begin{array}{ll}
\mu(\vec{r})=\sum_{i=1}^{p} g_{i}(\vec{r}) \chi_{\mathscr{D}_{i}}(\vec{r}) & \left(\vec{r}=(x, y) \in \mathbb{R}^{2}\right), \\
\mu(\vec{r})=0 & \left(|\vec{r}| \geqslant R_{H}, \quad R_{H}<0.5, \quad p \leqslant I\right) .
\end{array}
$$

В этой формуле $g_{i} \in C^{\infty}$ и $\chi_{\mathscr{D}}-$ характеристическая функция области $\mathscr{D}$. Таким образом, функция $\mu$ может иметь разрывы первого рода на объединении $\cup \partial \mathscr{D}_{i}$ кривых $\partial \mathscr{D}_{i}$. Здесь и везде ниже $\partial \mathscr{D}_{i}-$ граница $\mathscr{D}$.

Будем говорить, что $\mu \in\left\{\mu^{\omega}, I\right\}$, если $\mu$ имеет вид (1.1), $p \leqslant I$ и кривье $\partial \mathscr{D}_{i}$ - аналитические. Везде ниже предполагается, что $\mu \in\left\{\mu^{\omega}, I\right\}$. Заметим, что это предположение связано только с желанием максимально упростить формулировки и доказательства, и используемьй метод позволяет рассмотреть случай, когда кривые $\partial \mathscr{D}_{i}$ состоят из конечного числа дуг ограниченной гладкости, в предположении, что каждая дуга является либо отрезком прямой, либо имеет конечное число точек уплощения конечного порядка. Краткие замечания по этому поводу содержатся в конце $\S 5$.

Работа выполнена при финансовой поддержке Российского фонда фундаментальных исследований (грант № 96-01-00767). 
Число $I$, входящее в определение класса $\left\{\mu^{\omega}, I\right\}$, предполагается фиксированным. Так как все рассматриваемые ниже алгоритмы линейны, то достаточно рассмотреть случай $\mu=g \chi_{\mathscr{D}}$.

Везде ниже

$$
\begin{aligned}
\vec{a} & =\left(a_{x}, a_{y}\right), \quad a=|\vec{a}|=\sqrt{a_{x}^{2}+a_{y}^{2}} \\
\langle\vec{a}, \vec{b}\rangle & =a_{x} b_{x}+a_{y} b_{y}, \quad[\vec{a}, \vec{b}]=a_{x} b_{y}-a_{y} b_{x}
\end{aligned}
$$

и через $\widehat{f}$ обозначается преобразование Фурье функции $f$ :

$$
\begin{gathered}
\widehat{f}(\omega)=\int e^{i \omega t} f(t) d t, \quad \widehat{\mu}(\vec{\omega})=\iint e^{i \lambda\langle\vec{r}, \vec{\eta}\rangle} \mu(\vec{r}) d x d y \\
(\vec{\omega}=\lambda \vec{\eta}, \quad \lambda=|\vec{\omega}|, \quad \vec{\eta}=(\cos \varphi, \sin \varphi)) .
\end{gathered}
$$

Для функции $\mu \in\left\{\mu^{\omega}, I\right\}$ определено ее преобразование Радона

$$
J(\varphi, q)=\int \mu\left(q \vec{\eta}+t \vec{\eta}^{*}\right) d t \quad\left(\vec{\eta}^{*}=(-\sin \varphi, \cos \varphi)\right) .
$$

Здесь и ниже, если предель интегрирования или суммирования опущены, то оно производится от $-\infty$ до $+\infty$ и понимается в смысле главного значения. Ясно, что $J(\varphi, q)$ - интеграл от $\mu$ вдоль прямой $(\varphi, q)$, задаваемой уравнением $\langle\vec{r}, \vec{\eta}\rangle=q$. Функцию $p_{\varphi}\left(p_{\varphi}(q)=J(\varphi, q)\right)$ в литературе называют проекцией под углом $\varphi$. При $\mu=\left\{\mu^{\omega}, I\right\}$ функция $p_{\varphi}-$ кусочно-гладкая и $p_{\varphi}^{(1)} \in L^{1}$.

Сформулируем простейшую задачу восстановления, которая и решается ниже.

Рассмотрим сеть прямы $\left\{\varphi_{k}, q_{j}\right\}_{1}$, задаваемых уравнениями

$$
\begin{gathered}
q_{j}=j \Delta q+\delta, \quad \varphi_{k}=k \Delta \varphi, \quad \Delta \varphi=\frac{\pi}{N_{\varphi}}, \quad 0 \leqslant \delta<\Delta q, \\
k=0,1, \ldots, 2 N_{\varphi}-1, \quad j=0, \pm 1, \pm 2, \ldots
\end{gathered}
$$

Сеть $\left\{\varphi_{k}, q_{j}\right\}_{1}$ отвечает томографу первого поколения; более современные томографы отличаются, главньм образом, выбором исходной сети прямых, вдоль которых производится измерение исходных данных. Будем считать, что в качестве исходных данных выступают значения $Y_{j k}$ сглаженного преобразования Радона:

$$
Y_{j k}=J^{\Pi}\left(\varphi_{k}, q_{j}\right), \quad J^{\Pi}(\varphi, q)=\int \Pi\left(q-q^{\prime}\right) J\left(\varphi, q^{\prime}\right) d q^{\prime} .
$$

Функция П $(q)$ называется приборной функиией и является некоторой регуляризацией $\delta$-функции:

$$
\Pi(q)=h^{-1} \Pi_{1}\left(\frac{q}{h}\right), \quad \int \Pi(q) d q=1, \quad \Pi(q) \rightarrow \delta(q) \quad \text { при } h \rightarrow 0 .
$$


Предполагается, что функция П $(q)$ достаточно быстро убьвает при $|q| \rightarrow \infty$. Точное описание класса $\{\Pi\}$ рассматриваемьх приборных функций будет дано ниже. Примерами приборных функций являются

$$
\Pi_{1}(q)=h^{-1} \cdot\left\{\begin{array}{ll}
1 & \left(|q|<\frac{h}{2}\right), \\
0 & \left(|q|>\frac{h}{2}\right),
\end{array} \quad \Pi_{2}(q)=\frac{1}{h \sqrt{\pi}} e^{-q^{2} / h^{2}} .\right.
$$

На практике величина $\Delta \varphi$ выбирается согласованно с $\Delta q$. Естественное условие согласования имеет вид $\Delta q \simeq R_{0} \Delta \varphi$, где $R_{0} \gtrsim R_{H}$ - радиус области восстановления. Это условие может быть обосновано с помощью результатов, изложенных в $\S 3$. В связи с этим и чтобы иметь возможность рассматривать предел $\Delta \varphi \rightarrow 0, \Delta q \rightarrow 0$, будем говорить, что задан полный набор данных восстановления, если задань величины $Y_{j k}, \Delta q=L \Delta \varphi, L \geqslant L_{0}$ и $L_{0}$ не зависит от $\Delta q$.

Задача состоит в том, чтобы по полному набору данных $\left\{Y_{j k}\right\}$ приближенно восстановить функщию $\mu \in\left\{\mu^{\omega}, I\right\}$ при $|\vec{r}| \leqslant R_{0}<0.5$.

Усложнение этой формулировки, связанное с конечностью набора данных и наличием статистических ошибок (шума) кратко обсуждается ниже.

Алгоритм восстановления - это линейное отображение, позволяющее по набору $\left\{Y_{j k}\right\}$ исходных данных построить приближение $\mu^{A}$ функции $\mu$, задающее томограмму $\mu^{A}(\vec{r})$

$$
A\left(\left\{Y_{j k}\right\}\right)=\mu^{A}(\vec{r}) \quad\left(|\vec{r}| \leqslant R_{0}<0.5\right) .
$$

В такой постановке мы ограничиваемся линейными моделями с полным набором данных, и вне нашего рассмотрения остаются задачи с неполными данными $\left(\varphi_{k}<2 \pi-\Delta\right)$ и задачи о нелинейных эффектах в томографии, представляюшие большой теоретический и практический интерес [5]-[7], [13], [14].

При наличии полного набора данных алгоритм $A(1.7)$ естественно строить на основе формульи обращения преобразования Радона [15], [16]

$$
\begin{aligned}
\mu(\vec{r}) & =(P \Psi)(\vec{r})=\int_{0}^{\pi} \Psi(\varphi,\langle\vec{r}, \vec{\eta}\rangle) d \varphi \\
\Psi(\varphi, a) & =\frac{1}{2 \pi^{2}} \int_{0}^{\infty} t^{-2}[2 J(\varphi, a)-J(\varphi, a+t)-J(\varphi, a-t)] d t .
\end{aligned}
$$

В такой форме эта формула представляет собой суперпозищию операций свертки $J$ как функции $q$ с обобщенной функцией $-\left(2 \pi^{2}\right)^{-1} q^{-2}[17]$ и обратного проецирования P. На негладких функщиях формула (1.8) теряет смысл, и для построения алгоритма используется ее регуляризованный вариант

$$
\begin{aligned}
\widetilde{\mu}^{A}(\vec{r}) & =\frac{1}{2} \int_{0}^{2 \pi} \widetilde{\Psi}^{A}(\varphi,\langle\vec{r}, \vec{\eta}\rangle) d \varphi, \\
\widetilde{\Psi}^{A}(\varphi, a) & =\int H^{A}(a-q) J^{\Pi}(\varphi, q) d q,
\end{aligned}
$$

где $H^{A}(s)$ - некоторая регуляризация обобщенной функции $-\left(2 \pi^{2}\right)^{-1} s^{-2}$. Применяя к этим интегралам простейшую квадратурную формулу трапеций, получим класс 
$\{A\}$ алгоритмов, зависяших от выбора функции $H^{A}$. При $A \in\{A\}$ функиия $\mu^{A}=$ $A\left(\left\{Y_{j k}\right\}\right)$ восстанавливается по формулам

$$
\begin{gathered}
\mu^{A}(\vec{r})=\frac{1}{2} \sum_{k=0}^{2 N_{\varphi}-1} \Psi\left(\varphi_{k},\left\langle\vec{\eta}_{k}, \vec{r}\right\rangle\right) \quad\left(\vec{\eta}_{k}=\left(\cos \varphi_{k}, \sin \varphi_{k}\right)\right), \\
\Psi^{A}\left(\varphi_{k}, a\right)=\sum_{j} H^{A}\left(a-q_{j}\right) Y_{j k} \Delta q .
\end{gathered}
$$

Алгоритмы такого типа называются алгоритмами свертки и обратной проекиии ( АСОП). Именно эти алгоритмы используются в практике томографического восстановления и рассматриваются ниже.

С формальной точки зрения на этом заканчивается построение ММТ.

Наличие полного набора данных и выбор АСОП и составляет содержание термина двумерная радоновская томография.

Дальнейшие уточнения связаны с более детальным рассмотрением интересуюших нас алгоритмов. Прежде всего заметим, что переход к более сложным квадратурным формулам при вычислении интеграла $\widetilde{\Psi}^{A}(1.9)$, если эти формулы основаны на локальном методе приближения (см. ниже) и инвариантны относительно сдвига на $\Delta q$, приводит просто к переопределению функции $H^{A}$. Требования локальности и инвариантности естественны, если рассматривается случай малых $\Delta \varphi, \Delta q$. Учитывая, что преобразование Фурье обобщенной функции $-\left(2 \pi^{2}\right)^{-1} s^{-2}$ равно $(2 \pi)^{-1}|\lambda|$ (см. [17]), можно предположить, что

$$
H^{A}(s)=\frac{1}{4 \pi^{2}} \int e^{-i \lambda s}|\lambda| R^{A}(\lambda) d \lambda .
$$

Функция $R^{A}$ назьвается эффективным регуляризатором и зависит от параметра регуляризации $\Lambda^{-1}$,

$$
\begin{gathered}
R^{A}(\lambda) \rightarrow 1 \quad(\Lambda \rightarrow \infty), \quad R^{A}(\lambda)=1+O(|\lambda|) \quad(|\lambda| \rightarrow 0), \\
R^{A}(\lambda) \rightarrow 0 \quad(|\lambda| \rightarrow \infty) .
\end{gathered}
$$

Ниже рассматриваются только однородные регуляризаторы вида

$$
R^{A}(\lambda)=R_{1}^{A}\left(\frac{\lambda}{\Lambda}\right) .
$$

Наиболее важным обстоятельством при построении алгоритма является то, что с практической точки зрения интересны только алгоритмы высокого разрешения. Это алгоритмы, позволяюшие локализовать разрьвы функции $\mu$ с точностью $\sim \Delta q$. Требование высокого разрешения приводит к следуюшим условиям (см. [12]):

$$
\Lambda^{-1}=C \Delta q, \quad L \Delta \varphi=\Delta q, \quad h=C \Delta q \quad(C \simeq 1) .
$$

В соответствии с этим ниже рассматриваются только алгоритмы $A \in\{A\}$, для которых выполняются следуюшие условия однородности:

$$
H^{A}(s)=\Omega S^{A}(\xi), \quad \widehat{\Pi}(\lambda)=S_{\Pi}(\xi) \quad\left(\xi=\frac{\lambda}{\Omega}\right) .
$$


Здесь и везде ниже

$$
\Omega=\frac{\pi}{\Delta q} .
$$

Функции $S^{A}, S_{\Pi}$ в $(1.13)$ не зависят от $\Omega, S_{\Pi}$ - гладкая функция, $S^{A}-$ кусочно-гладкая функция и обе они убьвают при $|\xi| \rightarrow \infty$,

$$
S^{A}(\xi)=O\left(|\xi|^{-\varkappa_{1}}\right), \quad S_{\Pi}(\xi)=O\left(|\xi|^{-\varkappa_{2}}\right) \quad(|\xi| \rightarrow \infty)
$$

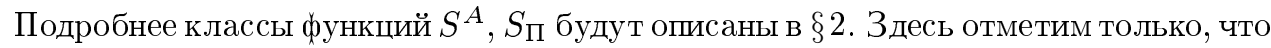
функция $S^{A}$ может иметь достаточно сложньй вид и, например, для используемых на практике алгоритмов

$$
S^{A}(\xi)=\frac{1}{2 \pi}\left(\frac{\sin (\pi \xi / 2)}{\pi \xi / 2}\right)^{p} \sum_{n}|\xi+2 p| S_{R}(\xi+2 p),
$$

где функция $S_{R}$ достаточно быстро убьвает и

$$
S_{R}(2 n)=0 \quad(n= \pm 1, \pm 2, \ldots) .
$$

Чтобы объяснить происхождение формулы (1.15) и понять приводимое в $\S 2$ определение алгоритма, рассмотрим конкретные примеры. Простейший класс алгоритмов без интерполяиии задается регуляризатором $R(\lambda)=S_{R}(\xi) \quad(\xi=\lambda / \Omega)$ и в этом случае $\left(A=A_{\infty}(R)\right)$

$$
H^{A}(s)=H_{R}(s)=\frac{1}{4 \pi^{2}} \int|\lambda| R(\lambda) e^{-i \lambda s} d \lambda .
$$

Алгоритмы без интерполящии не используются на практике, что связано с трудностью их машинной реализации (см. [5], [12]). Все используемые алгоритмы принадлежат к классу $A_{\alpha}(R, V)$ алгоритмов с интерполяиией. Чтобы построить эти алгоритмы, начнем с простого замечания об интерполяции. Пусть заданы отсчеты $f_{n}=f(n \Delta t)$ функции $f$. Линейньй и инвариантньй относительно сдвигов на $\Delta t$ onepamop интерполяии $V^{\Delta t}$ задается соотношением

$$
\left(V^{\Delta t}\left(\left\{f_{n}\right\}\right)\right)(t)=\sum_{n} V_{\Delta t}(t-n \Delta t) f_{n} \Delta t,
$$

и функщия $V_{\Delta t}$ называется функиией интерполяции. При малых $\Delta t$ наибольший интерес представляют локальные методы интерполяции, для которых $V_{\Delta t}(t)=0$ при $|t|>p \Delta t$. Подчеркнем, что несмотря на терминологию, выполнение условий интерполящии $\left(V^{\Delta t}\left(\left\{f_{n}\right\}\right)\right)(n \Delta t)=f_{n}$ в общем не предполагается. Aлгоритм $A_{\alpha}(R, V)$ задается выбором регуляризатора $R$, челого числа $\alpha=1,2, \ldots$ и функиии интерполяиии $V_{\frac{\Delta q}{\alpha}}$. По определению для $A=A_{\alpha}(R, V)$

$$
\begin{gathered}
\mu^{A}(\vec{r})=\frac{1}{2} \sum_{k=0}^{2 N_{\varphi}-1}\left(V^{\frac{\Delta q}{\alpha}}\left(\left\{\Psi_{R}^{T}\left(\varphi_{k}, \frac{\Delta q}{\alpha} n\right)\right\}\right)\right)\left\langle\vec{r}, \vec{\eta}_{k}\right\rangle \Delta \varphi, \\
\Psi_{R}^{T}\left(\varphi_{k}, a\right)=\sum_{j} H_{R}\left(a-q_{j}\right) Y_{j k} \Delta q,
\end{gathered}
$$


где функция $H_{R}$ определена в (1.16). Таким образом, для реализации этого алгоритма необходимо вычислить отсчеты $\Psi_{R}^{T}\left(\varphi_{k}, n \Delta q / \alpha\right)$, а затем использовать оператор интерполяции $V^{\Delta q / \alpha}$ для вычисления обратной проекции. Алгоритмы $A_{\alpha}(R, V)$ принадлежат к классу $\{A\}$ и для них

$$
\begin{aligned}
& H^{A}(s)=V^{\frac{\Delta q}{\alpha}}\left(\left\{H_{R}\left(n \frac{\Delta q}{\alpha}\right)\right\}\right)(s)=\sum_{n} V_{\frac{\Delta q}{\alpha}}\left(s-\frac{n \Delta q}{\alpha}\right) H_{R}\left(\frac{n \Delta q}{\alpha}\right) \frac{\Delta q}{\alpha}, \\
& \widehat{H}^{A}(\lambda)=\frac{1}{2 \pi} \widehat{V}_{\frac{\Delta q}{\alpha}}(\lambda) \sum_{p}|\lambda+2 p \alpha \Omega| R(\lambda+p \alpha \Omega) .
\end{aligned}
$$

Если вьполняются условия $\sum_{p}|p| R(2 p \alpha \Omega)=0$, то для $A=A_{\alpha}(R, V)$ существует эффективньй регуляризатор

$$
R^{A}(\lambda)=|\lambda|^{-1} \widehat{V}_{\frac{\Delta q}{\alpha}}(\lambda) \sum_{p}|\lambda+2 p \alpha \Omega| R(\lambda+2 p \alpha \Omega) .
$$

На практике используются локальные методы интерполяиии и $\left(V_{\Delta q}(q)=0\right.$, $|q|>p \Delta q)$ с малым $\alpha=1, \ldots, 4$. Чаше всего используются интерполяции нулевого или первого порядка (линейная интерполяция), для которых $V_{\Delta t}=V_{\Delta t}^{0,1}$,

$$
V_{\Delta t}^{0}(t)=\frac{1}{\Delta t} \cdot\left\{\begin{array}{ll}
1 & \left(|t|<\frac{\Delta t}{2}\right), \\
0 & \left(|t|>\frac{\Delta t}{2}\right),
\end{array} \quad V_{\Delta t}^{1}(t)=\frac{1}{\Delta t} \cdot \begin{cases}1-\frac{t}{\Delta t} & (|t|<\Delta t), \\
0 & (|t|>\Delta t),\end{cases}\right.
$$

и преобразования Фурье этих функций отвечают значениям $p=1,2$ в общей формуле для интерполяции локальньми $B$-сплайнами:

$$
\widehat{V}_{\Delta t}(\lambda)=\left(\frac{\sin (\lambda \Delta t / 2)}{\lambda \Delta t / 2}\right)^{p} .
$$

Из (1.18) следует, что при $\alpha=1$ можно ограничиться рассмотрением финитных регуляризаторов, для которых $R(\lambda)=0(|\lambda|>\Omega)$.

Приведем примеры трех регуляризаторов, используемых на практике:

$$
\begin{gathered}
R_{i}(\lambda)=S_{i}(\xi), \quad S_{i}(\xi)=0 \quad(|\xi|>1, \xi=\lambda / \Omega), \\
S_{1}(\xi)=1, \quad S_{2}(\xi)=\frac{\sin (\pi \xi / 2)}{\pi \xi / 2}, \quad S_{3}(\xi)=\frac{\sin \pi \xi}{\pi \xi} \quad(|\xi|<1),
\end{gathered}
$$

и, например, для алгоритмов с линейной интерполящией при $\alpha=1, R=R_{i}$

$$
\begin{gathered}
\widehat{H}^{A}(\lambda)=\Omega S^{A}(\xi)=\frac{\Omega}{2 \pi}\left(\frac{\sin (\pi \xi / 2)}{\pi \xi / 2}\right)^{2} T_{i}(\xi), \\
T_{1}(\xi)=|\xi|(\bmod 2), \quad T_{2}(\xi)=\frac{2}{\pi}\left|\sin \frac{\pi \xi}{2}\right|, \quad T_{3}(\xi)=\frac{1}{\pi}|\sin \pi \xi|
\end{gathered}
$$

и $T_{i}$ - ограниченная функция с периодом 2 . Более подробно примеры алгоритмов рассмотрены в [12]. Обший метод их построения вытекает из теории вычисления сингулярных сверток, изложенной в [18]. 
При определении полного набора данных предполагалось, что точно известно бесконечное число значений $Y_{j k}(1.4)$. На практике известен только конечньй набор $Y_{j k}$ $\left(|j| \leqslant N_{q}\right)$ со статистической ошибкой $\Delta Y_{j k}$. Учет статистических ошибок не представляет трудности, если считать, что $\Delta Y_{j k}-$ независимые случайные величины с нулевым средним и дисперсией $\sigma_{J}^{2}\left(\varphi_{k}, q_{j}\right)$. В этом случае для дисперсии $\sigma_{\mu}^{2}(\vec{r})$ величины $\mu^{A}(\vec{r})$ имеет место следуюшая формула, справедливая при $\Omega \gg 1$ и естественных предположениях о гладкости функции $\sigma_{J}^{2}(\varphi, q)[12]$ :

$$
\begin{aligned}
& \sigma_{\mu}^{2}(\vec{r}) \simeq \sigma_{J}^{2}(\vec{r}) \Delta \varphi(\Delta q)^{-2} \int_{0}^{\infty}\left(S^{A}(\xi)\right)^{2} d \xi \\
& \sigma_{J}^{2}(\vec{r})=\pi^{-1} \int_{0}^{\pi} \sigma_{J}^{2}(\varphi,\langle\vec{r}, \vec{\eta}\rangle) d \varphi
\end{aligned}
$$

Из этой формулы, в частности, видно, что величина $\sigma_{\mu}^{2}(\vec{r})$ расходится при $\Delta q \rightarrow 0$ :

$$
\sigma_{\mu}^{2}(\vec{r}) \simeq C(A) \sigma_{J}^{2}(\vec{r})\left(\Delta q R_{0}\right)^{-1} \quad\left(R_{0} \Delta \varphi \simeq \Delta q\right)
$$

Это общее свойство неустойчивости алгоритмов высокого разрешения, которые, тем не менее, используются на практике. Ниже статистические ошибки больше не рассматриваются.

Рассмотрим случай конечного набора данных, когдавеличины $Y_{j k}$ известны только при $|j| \leqslant N_{q}$. Через $\mu_{N_{q}}^{A}(\vec{r})$ обозначим результат восстановления с помошюю алгоритма $A$, если по определению считать, что $Y_{j k}=0$ при $|j|>N_{q}$, т.е. если суммирование по $j$ в (1.10) ведется по области $|j| \leqslant N_{q}$. Предположим, что

$$
N_{0} \gamma<N_{q} \quad\left(N_{0}=\frac{R_{0}}{\Delta q}, \gamma<1\right), \quad \Pi(q) \leqslant \frac{C}{\Delta q}\left(\frac{q}{\Delta q}\right)^{-m}
$$

Так как $\left|H^{A}(s)\right| \leqslant \bar{H}(\Delta q)^{-2}$ при $A \in\{A\}$, то

$$
\begin{gathered}
\left|\mu^{A}(\vec{r})-\mu_{N_{q}}^{A}(\vec{r})\right| \leqslant \frac{C M \bar{H}(\Delta q)^{m-2}}{(m-1)(1-\gamma)^{m} R_{0}^{m}}, \\
M=\max _{\varphi} \int|J(\varphi, q)| d q .
\end{gathered}
$$

Таким образом, для быстро убьвающих приборных функиий $(m \gg 1) u \Delta q \ll 1$ можно ограничиться рассмотрением величины $\mu^{A}(\vec{r})$, что и делается ниже.

Для алгоритмов высокого разрешения при выполнении условий однородности (1.13) результат восстановления $\mu^{A}(\vec{r})$ зависит от одного малого параметра $\Delta q$ и можно ставить вопрос о сходимости алгоритма при $\Delta q \rightarrow 0$.

Будем говорить, что алгоритм $А$ сходится в точке $\vec{r}(|\vec{r}|<0.5)$ для заданного $\mu \in\left\{\mu^{\omega}, I\right)$, если

$$
\begin{gathered}
\left|\Delta \mu^{A}(\vec{r})\right|=\left|\mu^{A}(\vec{r})-\mu(\vec{r})\right|=O\left(\Omega^{-\sigma_{\infty}^{A}(\vec{r})}\right) \quad(\Omega \rightarrow \infty), \\
\sigma_{\infty}^{A}(\vec{r})>0 .
\end{gathered}
$$


Далее величина

$$
\Delta \mu^{A}(\vec{r})=\mu^{A}(\vec{r})-\mu(\vec{r})
$$

называется ошибкой восстановления, а величина $\sigma_{\infty}^{A}(\vec{r})$ - асимптотическим показателем сходимости в точке $\vec{r}$. Как следует из этого определения, нас будет интересовать только поточечная сходимость. Для разрьвных функций $\mu$ такая сходимость не может быть равномерной, так как $\mu^{A}(\vec{r})$ - гладкая функция.

Хотя сходимость алгоритма зависит только от поведения величины $\mu^{A}(\vec{r})$ при $\Omega \rightarrow \infty$, в основном тексте рассматривается более сложная задача об оценке величины $\Delta \mu^{A}(\vec{r})$ при конечном $\Omega$. В этом случае также можно ввести понятие показателя сходимости $\sigma^{A}(\vec{r})$.

Пусть можно указать параметры $t_{1}, \ldots, t_{N}$, от которых зависит оценка сверху величинь $\left|\Delta \mu^{A}(\vec{r})\right|$. Тогда будем говорить, что для заданного $\mu \in\left\{\mu^{\omega}, I\right\}$ в точке $\vec{r}$ алгоритм $A$ имеет показатель сходимости $\sigma^{A}(\vec{r})$, если при $\Omega \geqslant$ $\Omega_{0}\left(t_{1}, \ldots, t_{N}\right)$

$$
\left|\Delta \mu^{A}(\vec{r})\right| \leqslant C\left(\vec{r}, t_{1}, \ldots, t_{N}\right) \Omega^{-\sigma^{A}(\vec{r})}, \quad \sigma^{A}(\vec{r})>0
$$

и константы $\Omega_{0}, C\left(\vec{r}, t_{1}, \ldots, t_{N}\right)$ ограниченьл и могут быть явно указаны.

Вопрос о существовании сходящихся алгоритмов высокого разрешения не является тривиальным даже в случае гладких $\mu$, так как речь, по-сушеству, идет о построении сходяшихся квадратурных формул для интеграла $\widetilde{\Psi}^{A}(\varphi, a)(1.9)$, в котором подьнтегральная функция сингулярным образом зависит от шага дискретизации $\Delta q$. Это частный случай задачи вычисления сингулярной свертки с помошњю алгоритма высокого разрешения [18]. В этой работе получены необходимые и достаточные условия сходимости таких алгоритмов. В интересующем нас случае эти условия имеют вид

$$
R^{A}(2 n \Omega)=0 \quad(n= \pm 1, \pm 2, \ldots) .
$$

Ниже случай гладких $\mu$ не рассматривается. Этот случай рассмотрен в работе [19], где доказана

ТеОРема 1.1 [19]. Пусть $\mu \in C^{\infty} \cap\left\{\mu^{\omega}, I\right\}$, выполняются условия однородности (1.13) и в некоторой окрестности $|\xi-2 p|<a$ точек $\xi=2 p \quad(p=1,2, \ldots)$ функция $S^{A}(1.13)$ имеет вид $S^{A}(\xi)=|\xi-2 p| a_{p}(\xi)+b_{p}(\xi)$, где $a_{p}, b_{p}$ - гладкие

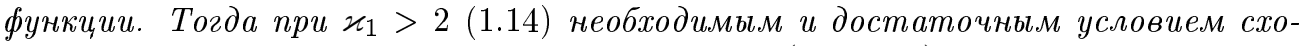
димости алгоритма $A \in\{A\}$ во всех точках $\vec{r}(|\vec{r}|<0.5)$ является выполнение одного из двух әквивалентных условий:

1) $a_{0}(0)=(2 \pi)^{-1}, b_{0}(0)=0, b_{p}(2 p)=0 \quad(p=1,2, \ldots)$;

2) существует эффективный регуляризатор $R^{A}(1.11)$, и для него выполняются условия (1.24).

В соответствии с этим везде ниже предполагается, что

$$
S^{A}(2 p)=0 \quad(p=1,2, \ldots) .
$$


Задача оценки показателя сходимости $\sigma^{A}(\vec{r})(1.23)$ эквивалентна задаче об оценке точности томографического восстановления. В данной работе рассматривается только случай $\mu=\chi_{\mathscr{D}}$. Более обший случай $\mu=g_{\mathscr{D}}$ рассмотрен в работе [19], где изложен метод разложения по гладкости, позволяюший результаты, полученные для $\mu=\chi_{\mathscr{D}}$, перенести на класс $\left\{\mu^{\omega}, I\right\}$. Из результатов работы [19] следует, что минимум показателя сходимости для класса $\left\{\mu^{\omega}, I\right\}$ достигается на функциях вида $\mu=\chi \mathscr{D}$ (см. конец $\S 5$ ).

Близкая к рассмотренной выше постановка задачи о сходимости, по-сушеству, содержится в книге [5]. Единственной известной нам работой (кроме работ автора, о которых речь пойдет ниже), где доказываются результаты о поточечной сходимости алгоритмов $A \in\{A\}$ при восстановлении разрывных функций, является работа [20], выполненная под руководством В.П. Паламодова. Краткое изложение этой работы опубликовано в [13], [21].

Чтобы сформулировать полученные в [20] результаты, введем некоторые определения, которые будут использоваться на протяжении всей работы. Вьше были определены величины $\mu^{A}, \widetilde{\mu}^{A}(1.10),(1.9)$. Величину

$$
\Delta \mu_{R}^{A}(\vec{r})=\widetilde{\mu}^{A}(\vec{r})-\mu(\vec{r})
$$

будем называть ошибкой регуляризачии. Введем величины

$$
\begin{aligned}
\mu_{q}^{A}(\vec{r}) & =\frac{1}{2} \int_{0}^{2 \pi} \Psi^{A}(\varphi,\langle\vec{r}, \vec{\eta}\rangle) d \varphi, \\
\Delta \mu_{q}^{A}(\vec{r}) & =\mu_{q}^{A}(\vec{r})-\widetilde{\mu}^{A}(\vec{r}) .
\end{aligned}
$$

Величину $\Delta \mu_{q}^{A}(\vec{r})$ называют ошибкой дискретизачии по q, а величину

$$
\Delta \mu_{T}^{A}(\vec{r})=\mu^{A}(\vec{r})-\widetilde{\mu}^{A}(\vec{r})
$$

- (полной) ошибкой дискретизации. Таким образом,

$$
\begin{aligned}
& \Delta \mu^{A}(\vec{r})=\mu^{A}(\vec{r})-\mu(\vec{r})=\Delta \mu_{T}^{A}(\vec{r})+\Delta \mu_{R}^{A}(\vec{r}), \\
& \Delta \mu_{T}^{A}(\vec{r})=\Delta \mu_{q}^{A}(\vec{r})+\Delta \widetilde{\mu}_{\varphi}^{A}(\vec{r})
\end{aligned}
$$

и последнее равенство можно считать определением величины $\Delta \tilde{\mu}_{\varphi}^{A}(\vec{r})$. Везде ниже $d=d\left(\vec{r}_{0}, \partial \mathscr{D}\right)-$ расстояние от точки $\vec{r}_{0}$ до границь $\partial \mathscr{D}$.

ТеОрема 1.2 [13], [21]. Пусть выполнены условия:

1) $\mu=g \chi \mathscr{D}, g \in C^{\infty}$ и гранииа $\partial \mathscr{D}$ - достаточно гладкая и строго выпуклая;

2) в алгоритме $A$ (1.10) функция $H^{A}$ получена линейной интерполячией отсчетов $H^{A}(j \Delta q)$, т.е. рассматривается алгоритм с интерполяиией (1.17), в котором $\alpha=1, V_{\Delta q}=V_{\Delta q}^{1}(q)$ (1.19);

3) $\sum_{j} H^{A}(j \Delta q)=0, S_{\Pi}(\xi)=\delta(\xi)$. 
Тогда при $d>\Delta q$ для величин $\Delta \mu_{q}^{A}\left(\vec{r}_{0}\right), \Delta \mu_{R}^{A}\left(\vec{r}_{0}\right)$ справедливь оценки:

$$
\left|\Delta \mu_{q}^{A}\left(\vec{r}_{0}\right)\right| \quad u \quad\left|\Delta \mu_{R}^{A}\left(\vec{r}_{0}\right)\right| \leqslant \begin{cases}C \Delta q d^{-1}+O(\Delta q) & \left(\vec{r}_{0} \in \mathscr{D}\right), \\ C(\Delta q)^{1 / 2} d^{-1 / 2}+O\left(\Delta q d^{-1 / 4}\right) & \left(\vec{r}_{0} \notin \mathscr{D}\right),\end{cases}
$$

а для $\Delta \widetilde{\mu}_{\varphi}^{A}\left(\vec{r}_{0}\right)$ nри всех d

$$
\left|\Delta \widetilde{\mu}_{\varphi}^{A}\left(\vec{r}_{0}\right)\right| \leqslant \begin{cases}C \Delta q L^{-1}(\Delta q+d)^{-1 / 2}+O\left(\Delta q L^{-1}\right) & \left(\vec{r}_{0} \in \mathscr{D}\right), \\ C(\Delta q)^{1 / 2} L^{-1}+O\left(\Delta q L^{-1}\right) & \left(\vec{r}_{0} \notin \mathscr{D}\right) .\end{cases}
$$

Заметим, что условие $\sum_{j} H^{A}(j \Delta q)=0$ эквивалентно (1.24) и рассматриваемый в теореме 1.2 алгоритм отвечает $p=2$ в (1.15). Метод, которым получены оценки $(1.30),(1.31)$, основан на прямом анализе соответствуюших ошибок в $q$-пространстве и не использует перехода в область Фурье-образов.

В работе [22] был предложен подход к рассматриваемой задаче, основанньй на использовании формулы Пуассона, позволяюший, по крайней мере на формальном уровне, получить асимптотически точные оценки ошибок восстановления. Основу этого подхода составляет точная формула, дающая при $\mu=\chi_{\mathscr{D}}$ представление $\mu^{A}\left(\vec{r}_{0}\right)$ в виде двойного ряда трехкратных осииллирующих интегралов (ОИ):

$$
\begin{aligned}
\mu^{A}\left(\vec{r}_{0}\right) & =\frac{i \Omega}{2 \pi} \sum_{n, m} J_{n m} e^{i 2 n \Omega \delta} \\
J_{n m} & =\int_{0}^{2 \pi} d \varphi \int_{0}^{\infty} d \xi \int_{\partial \mathscr{D}} d s e^{i \Omega \Phi_{n m}} u_{n} .
\end{aligned}
$$

В ОИ $J_{n m}$ фаза $\Phi_{n m}$ имеет вид

$$
\begin{gathered}
\Phi_{n m}(\varphi, \xi, s)=-2 L m \varphi+\langle\xi \vec{l}(s)+2 n \vec{r}(s), \vec{\eta}\rangle, \\
\vec{l}(s)=\vec{l}\left(\vec{r}_{0}, s\right)=\vec{r}(s)-\vec{r}_{0} \quad(\vec{r}(s) \in \partial \mathscr{D}) .
\end{gathered}
$$

Здесь и ниже $s$ - натуральный параметр, длина дуги на контуре $\partial \mathscr{D}$ с положительной

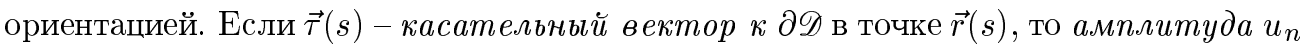
в ОИ $J_{n m}$ определяется соотношениями

$$
\begin{gathered}
u_{n}(\varphi, \xi, s)=v_{n}(\xi)[\vec{\tau}(s), \vec{\eta}], \\
v_{n}(\xi)=\frac{S^{A}(\xi) S_{n}^{\Pi}(\xi+2 n)}{\xi+2 n}, \quad S_{n}^{\Pi}(\xi)=\frac{1}{2}\left[S_{\Pi}(\xi)+e^{i 4 n \Omega \delta} S_{\Pi}(-\xi)\right] .
\end{gathered}
$$

Разложение (1.32) согласуется с представлениями (1.28), (1.29) в том смысле, что

$$
\Delta \mu_{T}^{A}\left(\vec{r}_{0}\right)=\frac{i \Omega}{2 \pi} \sum_{n, m}^{\prime} J_{n m} e^{i 2 n \Omega \delta}, \quad \Delta \mu_{q}^{A}\left(\vec{r}_{0}\right)=\sum_{n \neq 0} J_{n 0} e^{i 2 n \Omega \delta} .
$$

Штрих в сумме $\sum_{n, m}^{\prime}$ означает, что $(n, m) \neq(0,0)$.

Возможность достаточно полного анализа ОИ $J_{n m}(1.33)$ связана с тем, что критические точки фазы $\Phi_{n m}$ как функции двух переменных $\varphi, \xi$ - невырождены при $d>0$ 
(см. §4). Таким образом, все критические точки фазы $\Phi_{n m}$ имеют corank $\leqslant 1$ и принадлежат к классам $A_{k}[23]$. Это, в частности, позволяет дать формальную оценку ошибки дискретизации, получаюшуюся заменой ОИ $J_{n m}$ первым членом его асимптотического разложения при $\Omega \rightarrow \infty$. Сравнение формальной оценки с результатами численного моделирования дает удивительно хорошее совпадение, и формальный ответ полностью описывает геометрию артефактов. Apтефактами в томографии называется трансляционно-неинвариантная часть ошибки восстановления, медленно убываюшая с ростом $d\left(\vec{r}_{0}, \partial \mathscr{D}\right)$. Главньй вклад в величину артефактов, по крайней мере при не слишком малых $L(1.12)$, дает величина $\Delta \mu_{q}^{A}\left(\vec{r}_{0}\right)(1.27)$. Заметим, что по своему происхождению артефакты аналогичны "явлениям Гиббса", возникаюшим при замене ряда Фурье разрьвной функции его конечной суммой. Отсутствие трансляционной инвариантности связано с фиксацией сети прямых $\left\{\varphi_{k}, q_{j}\right\}_{1}(1.3)$, выделяющей начало координат - центр сканирования. Томограмма, представленная на рис. 1 , получена с помощью ММТ, описанной выше (подробнее см. [12]).
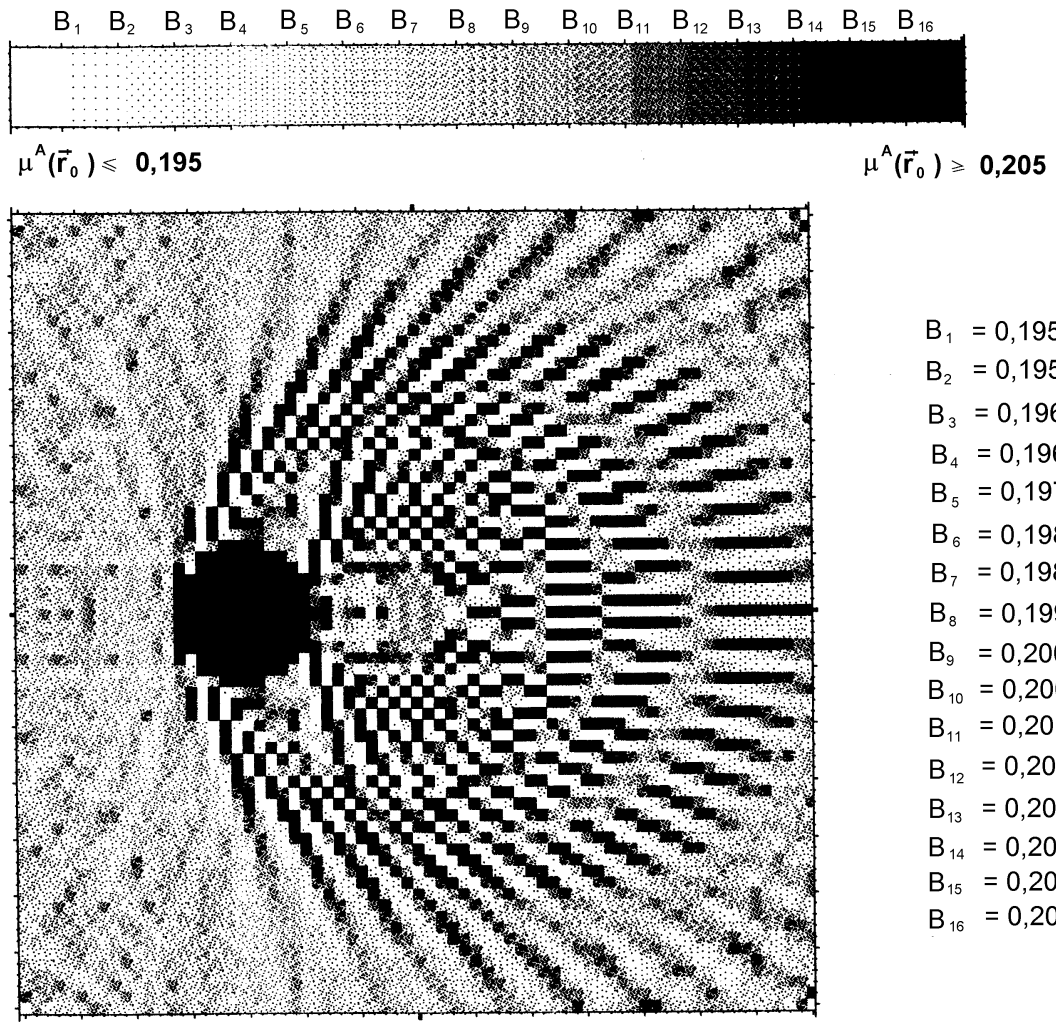

$B_{1}=0,19500000$

$\mathrm{B}_{2}=0,19566660$

$\mathrm{B}_{3}=0,19633320$

$\mathrm{B}_{4}=0,19699980$

$B_{5}=0,19766650$

$B_{6}=0,19833310$

$B_{7}=0,19899970$

$\mathrm{B}_{8}=0,19966630$

$B_{9}=0,20033290$

$B_{10}=0,20099960$

$B_{11}=0,20166620$

$B_{12}=0,20233280$

$\mathrm{B}_{13}=0,20299940$

$B_{14}=0,20366600$

$B_{15}=0,20433260$

$B_{16}=0,20500000$

Рис. 1

В качестве объекта выбрана функция $\mu(\vec{r})=0.2 \chi_{0}(\vec{r})+\chi_{1}(\vec{r})$, где $\chi_{0}-$ характеристическая функция круга радиуса $50 \Delta q$ с центром в начале координат, а $\chi_{1}$ - характеристическая функция круга радиуса $5 \Delta q$ с центром в точке $(-15 \Delta q, 0)$. Томограмма на рис. 1 отвечает приборной функции П $(q)=\Pi_{1}(q), h=\Delta q(1.6)$ и функции $S^{A}$, 
определенной равенством (1.15) при $p=2$ и $S_{R}=S_{1}$ (1.20). На рис. 1 представлен результат восстановления при $\Omega \simeq 300, L \simeq 0.5$ сеточной функции $\mu^{A}(\alpha \Delta q, \beta \Delta q)$ в квадрате со стороной $70 \Delta q$ и центром в начале координат. Маленькое деление на стороне квадрата отвечает $\Delta q$. Геометрия артефактов на рис. 1 описывается критическими точками фаз $\Phi_{n m}$ и связана с педальной кривой $[24]$, отвечающей линии разрыва функции $\chi_{1}$ (см. $\S 4$ и [12]).

Трудности обоснования формальной оценки связаны с необходимостью подстановки оценок индивидуальных интегралов $J_{n m}$ в ряд (1.32). Для обоснования возможности такой подстановки необходимо указать константы в оценках или, другими словами, иметь для ОИ $J_{n m}(1.33)$ оценки с константами. Первые строгие результаты в рамках этого подхода были получены в работе [19], где рассмотрен обший случай функций $\mu$ вида (1.1). В настоящее время развитие метода получения для ОИ оценок с константами [25] позволяет получить гораздо более полные, чем в работе [19], результаты. Этому и посвящена настоящая работа. Все необходимые результаты об оценках ОИ приведены в приложении. Доказательства большинства из них, но не всех, содержатся в [25].

В данной работе формальный ответ будет обоснован только для достаточно гладких алгоритмов. Точное определение этого понятия дано в $\S 2$. В частности, оно означает, что функция $S^{A}$ - достаточно гладкая, имеет нуль достаточно высокого порядка в точках $\xi=2 p(p=1,2, \ldots)$ и достаточно быстро убывает с ростом $|\xi|$. Ограничение этим случаем связано не столько с существом метода, сколько со стремлением максимально упростить доказательства и формулировки. В соответствии с этим везде будут использоваться самые грубые оценки, позволяюшие, тем не менее, получать асимптотически точные результаты. Полученные в $\S 5$ результаты не только асимптотически точнь, но и не могут быть улучшень за счет выбора алгоритма из класса $\{A\}$, кроме случая, когда $S^{A}(\xi)=0 \quad\left(|\xi|>a_{1}\right), S_{\Pi}(\xi)=0$ $\left(|\xi|>a_{2}\right), a_{1}+a_{2}<2$. Таким образом, ограничение классом достаточно гладких алгоритмов до некоторой степени оправдано тем, что для них получаются неулучшаемые на классе $\{A\}$ результаты. Результаты $\S 5$ уточняют оценки $(1.30),(1.31)$ и обобщают их на случай общих алгоритмов, невьпуклых контуров и $S_{\Pi}(\xi) \neq \delta(\xi)$. Тем не менее, необходимо еще раз подчеркнуть, что результаты $\S 5$ доказаны только для достаточно гладких алгоритмов при условии $d \geqslant C \Omega^{-\beta_{1}}\left(0<\beta_{1} \ll 1\right)$, тогда как оценки (1.30), (1.31) из работы [21] верны и без предположения достаточной гладкости при $d \sim \Delta q$. В связи с этим отметим, что точное поведение ошибки восстановления $\Delta \mu^{A}\left(\vec{r}_{0}\right)$ вблизи границы $\partial \mathscr{D}$ (при $d \sim \Delta q$ ) представляет большой практический интерес [12]. Этот вопрос в данной работе не рассматривается. Можно показать, что вблизи границы ошибки дискретизации не важны $\left|\Delta \mu_{T}^{A}\left(\vec{r}_{0}\right)\right| \leqslant C \Omega^{-\sigma}, \sigma>0$, тогда как ошибка регуляризации $\Delta \mu_{R}^{A}\left(\vec{r}_{0}\right)$ - порядка единицы и описывается при $\mu=\chi_{\mathscr{D}}$ некоторой универсальной функцией. Пусть $\vec{r}_{0}=\vec{r}(s)+\vec{\nu} x(x \simeq \Delta q)$ и $\vec{\nu}-$ внешняя нормаль к $\partial \mathscr{D}$ в точке $\vec{r}(s)$. Тогда

$$
\Delta \mu_{R}^{A}\left(\vec{r}_{0}\right)=\frac{1}{2}-\frac{1}{\pi} \int_{0}^{\infty} \lambda^{-1} R^{A}(\lambda) \widehat{\Pi}(\lambda) \sin \lambda x d \lambda+O\left(\frac{k(s)}{\Omega}\right),
$$

где $k(s)$ - кривизна $\partial \mathscr{D}$ в точке $\vec{r}(s)$ и главньй член в этой формуле не зависит от вида контура $\partial \mathscr{D}$. Выражение (1.36) хорошо описывает результаты численного эксперимента [12]. 
Список используемых обозначений приведен в конце приложения. Ссылки на формуль из приложения снабжаются буквой П.

\section{§. Формулировка результатов}

На протяжении всей работы используется

Соглашение о константах. Все константы, значения которых не указаны, обозначаются буквой $C$, и одной буквой могут обозначаться различные константыл. Всегда указывается, от каких величин зависят эти константыл. Если эти величины не указань, то константы - абсолютные. Если константы входят в условия справедливости некоторого утвержсдения или в само утверждение, то это означает, что существуют и могут быть явно указаны такие значения констант, что при выполнении соответствующих условий выполняется и утверждение.

Опишем область точек $\vec{r}_{0} \in \mathbb{R}^{2}$, в которой доказывается сходимость томографических алгоритмов и оцениваются ошибки дискретизации. Всегда предполагается, что $\vec{r}_{0} \in D, D=\left\{\vec{r}_{0} \in \mathbb{R}^{2}|| \vec{r}_{0} \mid<0.5\right\}$. Если $M \subset D$, то, по определению, $U(\beta, M)=\left\{\vec{r}_{0} \in D \mid d\left(\vec{r}_{0}, M\right) \leqslant C \Omega^{-\beta}, \beta>0\right\}$, и сходимость доказывается $\boldsymbol{\theta}$ области $D_{\beta_{1}}=D \backslash U\left(\beta_{1}, \partial \mathscr{D}\right)\left(\mu=\chi_{\mathscr{D}}\right)$. Эту область представим в виде объединения трех областей

$$
\begin{gathered}
D_{\beta_{1}}=\bigcup_{i=1}^{3} D_{\beta_{1} \beta_{2}}^{i}, \\
D_{\beta_{1} \beta_{2}}^{1}=\left(D_{\beta_{1}} \cap T(\partial \mathscr{D})\right) \backslash U\left(\beta_{2}, T_{0}\right), \\
D_{\beta_{1} \beta_{2}}^{2}=D_{\beta_{1}} \cap U\left(\beta_{2}, T_{0}\right), \quad D_{\beta_{1} \beta_{2}}^{3}=D_{\beta_{1}} \backslash\left(T(\partial \mathscr{D}) \cup U\left(\beta_{2}, T_{0}\right)\right) .
\end{gathered}
$$

Здесь $T(\partial \mathscr{D})$ - облединение всех касательных $\kappa \partial \mathscr{D}$, т.е. множество точек $\vec{r}_{0}$, из которых можно провести касательную к $\partial \mathscr{D}, T_{0}$ - обвединение касательньх $T\left(s_{i}^{0}\right)$, проведенных через точки уплощения $s_{i}^{0}$, и $D_{\beta_{1} \beta_{2}}^{2} \subset \bigcup_{i} U\left(\beta_{2}, T\left(s_{i}^{0}\right)\right)$. Геометрия областей $D_{\beta_{1} \beta_{2}}^{i}$ показана на рис. 2 .

Классы рассматриваемых приборных функций и алгоритмов, кратко описанные во введении, нуждаются в уточнении. Везде ниже функция одной переменной назьвается кусочно-гладкой, если она и все ее производные имеют только разрывы первого рода.

Относительно рассматриваемых приборных функиий П (1.5) будем предполагать, что они принадлежсат $\kappa$ классу $\{\Pi\}$. Это означает, что выполнень условия 1), 2):

1) П - кусочно-гладкая функиия, которая достаточно бьстро убывает:

$$
|\Pi(q)| \leqslant C(\Delta q)^{-1}\left(\frac{q}{\Delta q}\right)^{-m}, \quad m \gg 1
$$

2) выполнено условие однородности $\widehat{\Pi}(\lambda)=S_{\Pi}(\xi) \quad(\xi=\lambda / \Omega)$, функция $S_{\Pi}$ не зависит от $\Omega, S_{\Pi}(0)=1$ u

$$
\left|S_{\Pi}^{(k)}(\xi)\right| \leqslant C_{k} A_{1}, \quad\left|S_{\Pi}^{(k)}(\xi)\right| \leqslant C_{k} A_{2} e^{-\tau_{2}|\xi|}|\xi|^{-\varkappa_{2}}, \quad \tau_{2} \geqslant 0, \quad \varkappa_{2}>0 .
$$



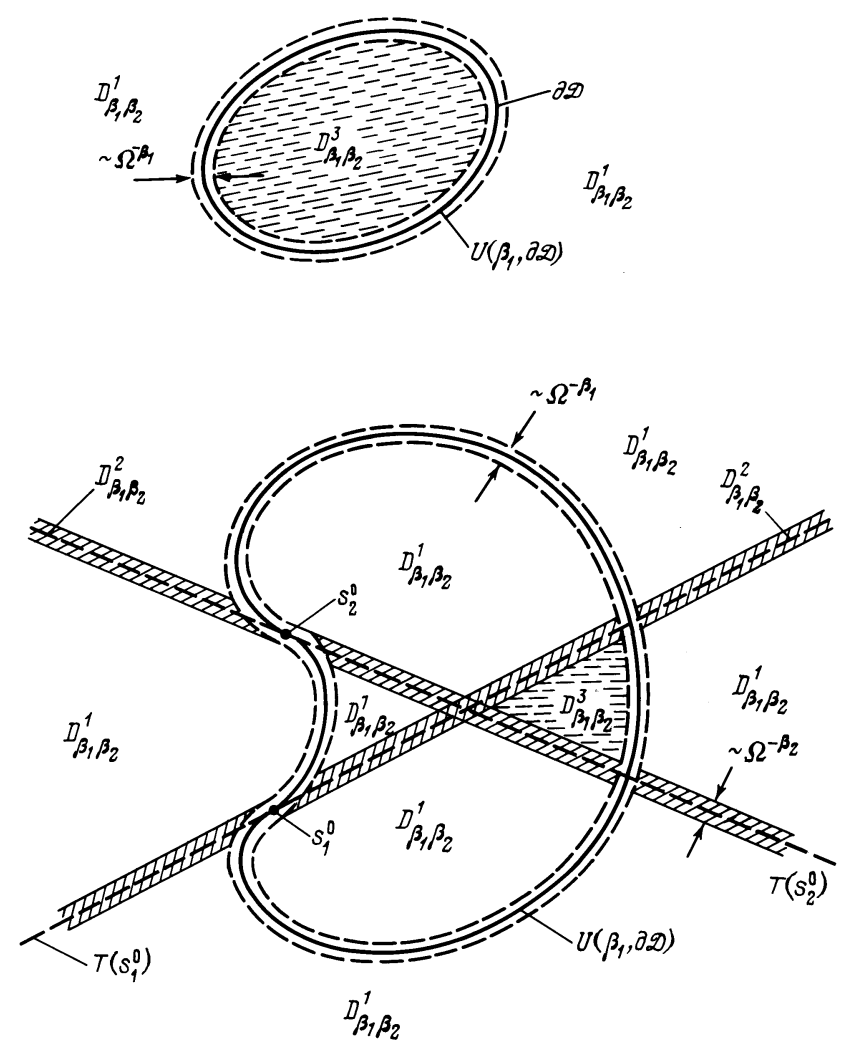

Рис. 2

Мотивировка следующего определения класса $\{A\}$ рассматриваемых алгоритмов содержится во введении. Будем говорить, что задан алгоритм $A \in\{A\}$, если задана четная, не зависящая от $\Omega$ функиия $S^{A}$, представимая в виде

$$
S^{A}(\xi)=P_{A}(\xi) T_{A}(\xi)=\frac{1}{2 \pi}|\xi| S_{R}^{A}(\xi)
$$

где $P_{A}, T_{A}, S_{R}^{A}$ - кусочно-гладкие функиии, имеющие не более счетного числа точек нарушения гладкости $\xi=\xi_{i}$,

$$
\left|\xi_{i}-\xi_{j}\right| \geqslant \Delta \xi \geqslant C \simeq 1,
$$

u такие, что

$$
\begin{gathered}
\left|P_{A}^{(k)}(\xi)\right| \leqslant C_{k} A_{3}, \quad\left|T_{A}^{(k)}(\xi)\right| \leqslant C_{k} A_{4}, \\
\left|\left(\frac{T_{A}(\xi)}{\xi-2 p}\right)^{(k)}\right| \leqslant C_{k} A_{5} \quad(p=0,1,2, \ldots), \quad\left|P_{A}^{(k)}(\xi)\right| \leqslant C_{k} A_{6} e^{-\tau_{1}|\xi|}|\xi|^{-\varkappa_{1}}, \\
\left|S_{R}^{A}(\xi)\right|=1+O(|\xi|) \quad(\xi \rightarrow 0), \quad S_{R}^{A}(2 p)=0 \quad(p=1,2, \ldots), \quad \tau_{1} \geqslant 0, \quad \varkappa_{1}>0 .
\end{gathered}
$$


Константы $A_{1}, A_{2}, \ldots, A_{6}$ нормированы таким образом, что $C_{0}=1$, и вектор $\vec{A}=\left(A_{1}, \ldots, A_{6}\right)$ характеризует алгоритм и приборную функцию.

Рассмотрим параметры, от значений которых зависят оценки ошибки дискретизации. Оказывается, что их число конечно, и все они будут сейчас перечислены.

Выбор алгоритма и массива данных задает константы $L, A_{0}$. Величина $L \geqslant L_{0}$ определена в (1.12) и $A_{0}=A_{0}(\vec{A})$,

$$
A_{0}=\max _{i, j, k}\left\{A_{i} \cdot A_{j} \cdot A_{k}\right\}
$$

Класс $\left\{\partial^{\omega}\right\}$ рассматриваемых контуров $\partial \mathscr{D}\left(\mu=\chi_{\mathscr{D}}\right)$ в сушности был определен выше, $\partial \mathscr{D} \in\left\{\partial \mathscr{D}^{\omega}\right\}$ - аналитический контур. В этом случае определены константы $k$, $b, K_{0}, \widetilde{k}$. Величины $k, b$ - это константы в оченках производных от кривизны:

$$
\left|k^{(p)}(s)\right| \leqslant \bar{k} b^{p}, \quad b \geqslant \bar{k} .
$$

Так как контур $\partial \mathscr{D} \in\left\{\partial \mathscr{D}^{\omega}\right\}$ может иметь только конечное число точек уплощения $s_{i}^{0}$ конечного порядка $p_{i}$, то

$$
\begin{gathered}
k^{(n)}\left(s_{i}^{0}\right)=0, \quad\left(0 \leqslant n \leqslant p_{i}-1\right), \quad\left|k^{\left(p_{i}\right)}\left(s_{i}^{0}\right)\right|=K_{i}>0, \\
K_{0}=\min _{i}\left\{K_{i}\right\}, \quad 1 \leqslant p_{i} \leqslant p,
\end{gathered}
$$

и величина $\widetilde{k}$ определяется из условия

$$
|k(s)| \geqslant \widetilde{k} \quad n p u \quad\left|s-s_{i}^{0}\right| \geqslant \Delta_{i} \equiv C_{i} \frac{K_{i}}{\bar{k} b^{p_{i}+1}} .
$$

Везде ниже $\vec{\nu}$ - внешняя нормаль $\kappa$ контуру ӘФ, который предполагается положительно ориентированным. В этом случае кривизна $k(s)$ может иметь оба знака, но вид уравнений Серре-Френе сохраняется [26]:

$$
\frac{d \vec{\tau}}{d s}=k \vec{\nu}, \quad \frac{d \vec{\nu}}{d s}=-k \vec{\tau}
$$

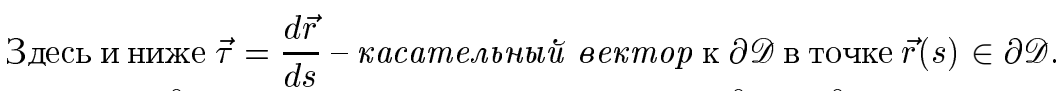

Пусть $\vec{r}_{m}^{0}-$ точки пересечения прямых $T\left(s_{i}^{0}\right) \quad\left(\left|\vec{r}_{m}^{0}\right| \leqslant 0.5\right)$. Через $\left\{\theta_{m}^{0}\right\}$ обозначим набор углов между этими прямыми, а через $d_{1}$ - минимальное расстояние между непересекающимися отрезками прямых $T\left(s_{i}^{0}\right)$, лежащими в круге D. Для оценки в области $D_{\beta_{1} \beta_{2}}^{1} \cup D_{\beta_{1} \beta_{2}}^{2}$ достаточно задать еще длину $\mathscr{L}$ контура $\partial \mathscr{D} u$

$$
\theta=\min \left\{\left|\vec{r}_{m}^{0}-\vec{r}_{m^{\prime}}^{0}\right|, \theta_{m}^{0}, d_{1}\right\}
$$

Для оценки в области $D_{\beta_{1} \beta_{2}}^{3}$, т.е. при $\vec{r}_{0} \notin T(\partial \mathscr{D})$, необходимо задать дополнительные параметры. Чтобы их ввести, рассмотрим функцию

$$
f(s)=\frac{d}{d s}\langle\vec{r}(s), \vec{\tau}(s)\rangle=1+k(s)\langle\vec{r}(s), \vec{\nu}(s)\rangle .
$$


Если $\mathscr{D}$ - окружность с центром в начале координат, то $f \equiv 0$. В остальных случаях функция $f$ имеет конечное число нулей конечного порядка

$$
f\left(s_{\alpha}\right)=0, \ldots, f^{\left(q_{\alpha}-1\right)}\left(s_{\alpha}\right)=0, \quad\left|f^{\left(q_{\alpha}\right)}\left(s_{\alpha}\right)\right|=f_{\alpha}>0 \quad\left(1 \leqslant q_{\alpha} \leqslant q\right) .
$$

Определим величины $\gamma_{\alpha}$ из условия

$$
\gamma_{\alpha}=\left\langle\vec{r}\left(s_{\alpha}\right), \vec{\tau}\left(s_{\alpha}\right)\right\rangle
$$

и будем предполагать, что

$$
\left|\gamma_{\alpha}-\gamma_{\alpha^{\prime}}\right| \geqslant \Delta^{0}
$$

Величина $\Delta^{0}$ является одним из трех новых параметров. Два других $-\widetilde{f}, f_{0}-$ определяются из условий

$$
\begin{gathered}
|f(s)| \geqslant \widetilde{f} \text { при }\left|s-s_{\alpha}\right| \geqslant C_{\alpha} f_{\alpha}\left(\bar{k} b^{q_{\alpha}+1}\right)^{-1}, \\
f_{\alpha} \geqslant f_{0} .
\end{gathered}
$$

Пронумеруем все введенные параметры:

$$
\begin{aligned}
& \left(\bar{k}, \quad b, \quad \mathscr{L}, \quad A_{0}, \quad K_{0}, \quad \widetilde{k}, \quad L, \quad f_{0}, \quad \tilde{f}, \quad \Delta^{0}, \quad \theta\right) \\
& =\left(t_{1}, \quad t_{2}, \quad t_{3}, \quad t_{4}, \quad t_{5}, \quad t_{6}, \quad t_{7}, t_{8}, t_{9}, t_{10}, t_{11}\right) \text {. }
\end{aligned}
$$

Непосредственно в оценку ошибки дискретизации $\Delta \mu_{T}^{A}\left(\vec{r}_{0}\right)(1.28)$ входят только некоторые из указанных параметров. Остальные необходимы для формулировки условий, при которых эта оценка справедлива. По тому, как эти параметры входят в условия, они разбиваются на две группы $-T_{1}, T_{2}$.

Скажем, что $t_{i} \in T_{1}$, если в условия входят только ограничения на $t_{i}$ сверху, u тогда по определению будем считать, что $t_{i} \geqslant 1$, если $t_{i} \in T_{1}$. Аналогично, $t_{i} \in T_{2}, t_{i} \leqslant 1$, если в условия входят только ограничения на $t_{i}$ снизу. В интересующем нас случае

$$
T_{1}=\left(t_{1}, t_{2}, t_{3}, t_{4}\right), \quad T_{2}=\left(t_{5}, t_{6}, t_{7}, t_{8}, t_{9}, t_{10}, t_{11}\right) .
$$

Осталось еще ввести понятие достаточно гладкого алгоритма. Кроме введенных вьше параметров $\vec{A}$ алгоритм характеризуется числом (см. (2.2), (2.5))

$$
\varkappa=\min \left\{\varkappa_{1}, \varkappa_{2}+1\right\} \text {, }
$$

которое задает скорость убывания функции $v_{n}(1.35)$ при $|\xi| \rightarrow \infty$.

Рассмотрим характеристики гладкости функиии $v_{n}$. Так как в ОИ (1.33) интегрирование ведется по положительной полуоси $\xi \geqslant 0$, то ниже по определению считается, что

$$
v_{n}(\xi)=0 \quad(\xi<0)
$$


Таким образом, точка $\xi=0$ всегда входит в множество $\left\{\xi_{i}^{n}\right\}$ точек нарушения гладкости функиии $v_{n}$. Также по определению считаем, что в это множество входят точки $\xi=2 p(p=1,2, \ldots)$. Числа $j_{i n}^{0}$ задают порядок нуля функции $v_{n}$ в точке $\xi_{i}^{n}$ :

$$
\begin{gathered}
v^{(p)}\left(\xi_{i}^{n}\right)=0 \quad\left(p \leqslant j_{i n}^{0}-1\right), \\
v_{n}^{\left(j_{i n}^{0}\right)}\left(\xi_{i}^{n}+0\right) \neq 0 \quad \text { или } \quad v_{n}^{\left(j_{i n}^{0}\right)}\left(\xi_{i}^{n}-0\right) \neq 0,
\end{gathered}
$$

а числа $j_{i n}^{1}$ определяют гладкость функции $v_{n}$ в этой точке:

$$
\begin{gathered}
v_{n}^{(p)}\left(\xi_{i}^{n}+0\right)=v_{n}^{(p)}\left(\xi_{i}^{n}-0\right) \quad\left(p \leqslant j_{i n}^{1}-1\right), \\
\Delta_{\xi_{i}^{n}} v_{n}^{\left(j_{i n}^{1}\right)} \equiv v_{n}^{\left(j_{i n}^{1}\right)}\left(\xi_{n}^{i}+0\right)-v_{n}^{\left(j_{i n}^{1}\right)}\left(\xi_{n}^{i}-0\right) \neq 0 .
\end{gathered}
$$

Будем говорить, что некоторое утверждение (оценка) справедливо для достаточно гладкого алгоритма, если можно указать числа ћо, $j_{0}, j_{1}$ такие, что оно верно при условиях $\tau_{1}=\tau_{2}=0$ и

$$
\varkappa \geqslant \varkappa_{0}, \quad j_{i n}^{1} \geqslant j_{1} \quad\left(\xi_{i}^{n} \neq 0\right),
$$

$$
j_{i n}^{0} \geqslant j_{0} \quad\left(\xi_{i}^{n}=-2 n, n=-1,-2, \ldots\right), \quad v_{0}^{(n)}(+0)=0 \quad\left(n=1, \ldots, j_{0}\right) .
$$

Через $s_{c}^{1} \equiv s_{c}^{1}\left(\vec{r}_{0}\right)$ обозначим критические точки первого типа фазы $\Phi_{n m}($ см. $\S 4)$. Для определения этих точек достаточно из точки $\vec{r}_{0}$ провести касательные к $\partial \mathscr{D}$, и тогда $\vec{r}\left(s_{c}^{1}\right) \in \partial \mathscr{D}$ - точки касания.

Введем величины

$$
\begin{aligned}
\xi_{n m}^{ \pm}\left(s_{c}^{1}\right) & \equiv \xi_{n m}^{ \pm}\left(s_{c}^{1}\left(\vec{r}_{0}\right), \vec{r}_{0}\right)=\mp \frac{2 L m}{l\left(s_{c}^{1}\right)}+2 n \frac{\left\langle\vec{r}\left(s_{c}^{1}\right), \vec{l}\left(s_{c}^{1}\right)\right\rangle}{l^{2}\left(s_{c}^{1}\right)} \varepsilon_{1}^{c}, \\
\vec{l}\left(s_{c}^{1}\right) & \equiv \vec{l}\left(\vec{r}_{0}, s_{c}^{1}\right)=\vec{r}_{0}-\vec{r}\left(s_{c}^{1}\right)=\varepsilon_{1}^{c} \vec{\tau}\left(s_{c}^{1}\right) l\left(s_{c}^{1}\right), \quad \varepsilon_{1}^{c}= \pm 1 .
\end{aligned}
$$

Для достаточно гладких алгоритмов ряды

$$
\begin{aligned}
& G_{i}\left(A, \vec{r}_{0}\right)=\sum_{(n, m) \neq(0,0)} \sum_{ \pm} \max _{s_{c}^{1}\left(\vec{r}_{0}\right)}\left\{\frac{\left|S^{A}\left(\xi_{n m}^{ \pm}\left(s_{c}^{1}\right)\right) S_{n}^{\Pi}\left(\xi_{n m}^{ \pm}\left(s_{c}^{1}\right)+2 n\right)\right|}{\left|\xi_{n m}^{ \pm}\left(s_{c}^{1}\right)+2 n\right|^{1+1 /(p+2)}}\right\} \\
& G_{0}\left(A, \vec{r}_{0}\right)=G_{i}\left(A, \vec{r}_{0}\right) \quad\left(p_{i}=0\right)
\end{aligned}
$$

сходятся равномерно по $\vec{r}_{0}$ и допускают оценки

$$
G_{i}\left(A, \vec{r}_{0}\right) \leqslant C A_{0} L^{-\varkappa}, \quad G_{0}\left(A, \vec{r}_{0}\right) \leqslant C A_{0} L^{-\varkappa} .
$$

Рассмотрим окрестности $U\left(\beta_{3}, \vec{r}_{m}^{0}\right)$ точек $\vec{r}_{m}^{0}$ пересечения прямых $T\left(s_{i}^{0}\right)$ и трубчатые окрестности $U\left(\beta_{2}, T\left(s_{i}^{0}\right)\right)$ прямых $T\left(s_{i}^{0}\right)$. Если $\theta>C \Omega^{-\alpha}$ и

$$
\beta_{2} \geqslant \alpha, \quad \beta_{2} \geqslant \beta_{3}+\alpha,
$$

то окрестности $U\left(\beta_{3}, \vec{r}_{m}^{0}\right)$ между собой не пересекаются и окрестности $U\left(\beta_{2}, T\left(s_{i}^{0}\right)\right)$ не пересекаются вне объединения кругов $U\left(\beta_{3}, \vec{r}_{m}^{0}\right)$. Таким образом, окрестности 
$U\left(\beta_{3}, \vec{r}_{m}^{0}\right), U\left(\beta_{2}, T\left(s_{i}^{0}\right)\right)$ образуют правильное, в смысле работы [25], покрытие множества $T_{0}=\bigcup T\left(s_{i}^{0}\right)$. При $\vec{r}_{0} \in D_{\beta_{1} \beta_{2}}^{2} \backslash \cup U\left(\beta_{5}, \vec{r}_{m}^{0}\right)$ имеется единственная окрестность $U\left(\beta_{2}, T\left(s_{i}^{0}\right)\right)$ такая, что $\vec{r}_{0} \in U\left(\beta_{2}, T\left(s_{i}^{0}\right)\right)$ и $\vec{r}_{0 i}-$ проекиия $\vec{r}_{0}$ на $T\left(s_{i}^{0}\right)$. Через $D^{\prime}=C \Omega^{-\beta_{3}}$ будем обозначать радиус круга $U\left(\beta_{3}, \vec{r}_{m}^{0}\right)$. В оценках при $\vec{r}_{0} \in$ $U\left(\beta_{2}, T\left(s_{i}^{0}\right)\right),\left|\vec{r}_{0}-\vec{r}_{m}^{0}\right| \geqslant D^{\prime}$ через $s_{0 c}^{1}$ обозначаются критические точки первого типа, $s_{0 c}^{1}=s_{c}^{1}\left(\vec{r}_{0 i}\right) \neq s_{i}^{0}$. Если в точке $\vec{r}_{m}^{0}$ пересекаются прямые $T\left(s_{i}^{0}\right)$, то миь будем записыв вать это в виде $i \subset(m)$. В оценках при $\left|\vec{r}_{0}-\vec{r}_{m}^{0}\right| \leqslant D^{\prime}=C \Omega^{-\beta_{3}}$ через $s_{0 c}^{1}$ обозначаются точки $s_{c}^{1}\left(\vec{r}_{m}^{0}\right) \neq s_{i}^{0}(i \subset(m))$.

TeOpema 2.1. Пусть $A \in\{A\}, \mu=\chi_{\mathscr{D}} \in\left\{\mu^{\omega}, I\right\} \quad\left(\partial \mathscr{D} \in\left\{\partial \mathscr{D}^{\omega}\right\}\right)$. Тогда можно указать числа $\alpha>0, \varepsilon_{0}>0, \beta_{i}>0 \quad(i=1,2,3), \varkappa_{0}, j_{0}, j_{1}$ такие, что при

$$
\begin{aligned}
\alpha_{i} & \leqslant \alpha, \quad \varepsilon \\
t_{i} \leqslant C_{i} \Omega^{\alpha_{i}} \quad\left(t_{i} \in T_{1}\right), \quad \varepsilon_{0}, & \\
t_{i} & \geqslant C_{i} \Omega^{-\alpha_{i}} \quad\left(t_{i} \in T_{2}\right)
\end{aligned}
$$

для любого достаточно гладкого алгоритма, т.е. при выполнении условий (2.22), справедливы следующие оченки ошибки восстановления:

1) при $\vec{r}_{0} \in D_{\beta_{1} \beta_{2}}^{1}$ имеет место оченка

$$
\begin{gathered}
\left|\Delta \mu^{A}\left(\vec{r}_{0}\right)\right| \leqslant C G_{0}\left(A, \vec{r}_{0}\right) \sum_{s_{c}^{1}\left(\vec{r}_{0}\right)}\left|\Omega k\left(s_{c}^{1}\right)\right|^{-1 / 2} l\left(s_{c}^{1}\right)^{-1}+R, \\
R \leqslant \gamma \frac{C_{1}\left(\varepsilon_{0}, \varkappa_{0}, j_{0}, j_{1}\right)}{\Omega^{0.5+\varepsilon}}+C_{2}\left(\varepsilon_{0}, \varkappa_{0}, j_{0}, j_{1}\right) A_{0} \mathscr{L} \Omega e^{-\gamma \Omega} \quad(\forall \gamma \geqslant 1) ;
\end{gathered}
$$

2) если $\vec{r}_{0} \in D_{\beta_{1} \beta_{2}}^{2}, \vec{r}_{0} \in U\left(\beta_{2}, T\left(s_{j}^{0}\right)\right) u\left|\vec{r}_{0}-\vec{r}_{m}^{0}\right| \geqslant D^{\prime}=C \Omega^{-\beta_{3}}, m o$

$$
\begin{aligned}
& \left|\Delta \mu^{A}\left(\vec{r}_{0}\right)\right| \leqslant C G_{0}\left(A, \vec{r}_{0 j}\right) \sum_{s_{0 c}^{1}}\left|\Omega k\left(s_{0 c}^{1}\right)\right|^{-1 / 2} l\left(\vec{r}_{0 j}, s_{0 c}^{1}\right)^{-1} \\
& \quad+C_{j} G_{j}\left(A, \vec{r}_{0 j}\right)\left|\Omega K_{j}\right|^{-1 /\left(p_{j}+2\right)} l\left(\vec{r}_{0 j}, s_{j}^{0}\right)^{-1}+C_{j}\left(\Omega K_{j}\right)^{-\frac{1}{p_{j}+2}-\varepsilon}+R,
\end{aligned}
$$

a npu $\vec{r}_{0} \in D_{\beta_{1} \beta_{2}}^{2} u\left|\vec{r}_{0}-\vec{r}_{m}^{0}\right| \leqslant D^{\prime} \quad\left(\vec{r}_{m}^{0} \in T\left(s_{i}^{0}\right), i \subset(m)\right)$

$$
\begin{aligned}
\left|\Delta \mu^{A}\left(\vec{r}_{0}\right)\right| \leqslant & C G_{0}\left(A, \vec{r}_{m}^{0}\right) \sum_{s_{0 c}^{1}}\left|\Omega k\left(s_{0 c}^{1}\right)\right|^{-1 / 2} l\left(\vec{r}_{m}^{0}, s_{0 c}^{1}\right)^{-1} \\
& +\sum_{i \subset(m)} C_{i} G_{i}\left(A, \vec{r}_{m}^{0}\right)\left|\Omega K_{i}\right|^{-\frac{1}{p_{i}+2}} l\left(\vec{r}_{m}^{0}, s_{i}^{0}\right) \\
& +\sum_{i \subset(m)} C_{i}\left(\Omega K_{i}\right)^{-\frac{1}{p_{i}+2}-\varepsilon}+R,
\end{aligned}
$$

где запись $i \subset(m)$ означает, что прямые $T\left(s_{i}^{0}\right)$ пересекаются в точке $\vec{r}_{m}^{0}$, и для $R$ справедлива та же оценка (2.28); 
3) если $\vec{r}_{0} \in D_{\beta_{1} \beta_{2}}^{3} u$ контур $\partial \mathscr{D}$ не является окружностью с чентром в начале координат, то $\forall \gamma \geqslant 1$ выполняется оченка

$$
\begin{gathered}
\left|\Delta \mu^{A}\left(\vec{r}_{0}\right)\right| \leqslant \gamma C_{q p}\left(\varepsilon_{0}, t_{i}\right) d_{T}^{-4} \Omega^{-3 / 2-1 /(q+2)}+R_{1}, \\
R_{1} \leqslant C_{1}\left(\varepsilon_{0}, \varkappa_{0}, j_{0}, j_{1}\right) \gamma \Omega^{-2}+C_{2}\left(\varepsilon_{0}, \varkappa_{0}, j_{0}, j_{1}\right) \mathscr{L} A_{0} \Omega e^{-\gamma \Omega}
\end{gathered}
$$

в которой можнно считать, что

$$
C_{q p}\left(\varepsilon_{0}, t_{i}\right)=C\left(\varepsilon_{0}\right) A_{0}(k)^{7 / 2} b^{q+1} \mathscr{L}^{2} \widetilde{K}_{p}^{-1 /(q+2)} L^{-1} f_{0}^{-4 / 3}
$$

В этих формулах величина q определена в (2.12), $p$ - в (2.8) и

$$
d_{T}=\min \left\{d\left(\vec{r}_{0}, \partial \mathscr{D}\right), d\left(\vec{r}_{0}, T_{0}\right)\right\}, \quad \widetilde{K}_{p}=\min \left\{C_{p} K_{0}^{p+1}(\bar{k})^{-p} b^{-p^{2}}, \widetilde{k}\right\}
$$

а если ӘФ - окружность с иентром в начале координат и радиусом $B$, то

$$
\left|\Delta \mu^{A}\left(\vec{r}_{0}\right)\right| \leqslant \gamma \frac{C\left(\varepsilon_{0}\right) B A_{0}}{\Omega^{3 / 2} d_{T}^{2} L}+R_{1} \quad(\forall \gamma \geqslant 1) .
$$

В конце $\S 5$ кратко обсуждается вопрос о том, как изменятся оценки величины $\Delta \mu^{A}\left(\vec{r}_{0}\right)$, если ослабить предположения теоремы 2.1 и вопрос об их точности. Из теоремы 2.1 , в частности, следует, что при $\mu \in\left\{\mu^{\omega}, I\right\}, d(\vec{r}, \partial \mathscr{D})>0$

$$
\sigma_{\infty}^{A}(\vec{r}) \geqslant \begin{cases}1 / 2 & \left(\vec{r} \in T(\partial \mathscr{D}), \vec{r} \notin T_{0}\right), \\ 1 /(p+2) & \left(\vec{r} \in \cap T\left(s_{i}^{0}\right) \quad\left(p=\max p_{i}\right)\right), \\ 3 / 2+1 /(q+2) & (\vec{r} \notin T(\partial \mathscr{D})),\end{cases}
$$

где $\sigma_{\infty}^{A}(\vec{r})$ - асимптотический показатель сходимости (1.21).

\section{§ 3. Ошибки восстановления и ряды осциллирующих интегралов}

Так как классическая формула обрашения (1.8) неприменима для восстановления функций типа $\mu=\chi_{\mathscr{D}}$, начнем с вывода формулы обрашения преобразования Радона на классе $\left\{\mu^{\omega}, I\right\}$.

ЛЕмма 3.1 (теорема о проекции; см. [5], [15]). Пусть $\mu \in\left\{\mu^{\omega}, I\right\}$. Тогда

$$
\widehat{\mu}(\lambda \vec{\eta})=\widehat{J}(\varphi, \lambda)
$$


ДокАЗАТЕЛЬСтво. По определению преобразования Радона

$$
\widehat{J}(\varphi, \lambda)=\int e^{i \lambda q} d q \int \mu\left(q \vec{\eta}+t \vec{\eta}^{*}\right) d t .
$$

При $\mu \in\left\{\mu^{\omega}, I\right\}$ этот интеграл сходится абсолютно и можно менять порядок интегрирования. Остается перейти от переменных $t, q$ к переменным $(x, y)=q \vec{\eta}+t \vec{\eta}^{*}$, чтобы получить (3.1).

В силу теоремы о проекции вопрос об обрашении преобразования Радона сводится к вопросу об обрашении двумерного преобразования Фурье. При $\vec{r}_{0} \in \cup \partial \mathscr{D}_{i}$ определим значение $\mu\left(\vec{r}_{0}\right)$ равенством

$$
\mu\left(\vec{r}_{0}\right)=\lim _{\varepsilon \rightarrow 0} \frac{1}{2 \pi} \int_{0}^{2 \pi} \mu\left(\vec{r}_{0}+\varepsilon \vec{\eta}\right) d \varphi
$$

и введем величину

$$
\mu_{\Lambda}(\vec{r})=\frac{1}{4 \pi^{2}} \int_{0}^{\pi} d \varphi \int_{-\Lambda}^{\Lambda}|\lambda| e^{-i \lambda\langle\vec{r}, \vec{\eta}\rangle} \widehat{J}(\varphi, \lambda) d \lambda,
$$

отвечающую суммированию интеграла Фурье по кругам [27], [28].

TeOpema 3.1. Пусть $\mu \in\left\{\mu^{\omega}, I\right\}$. Тогдa $\mu(\vec{r})=\lim _{\Lambda \rightarrow \infty} \mu_{\Lambda}(\vec{r})$.

ДокАЗАТЕЛЬСТво. В силу теоремы 1.32 из [28] достаточно доказать, что функция $h_{\vec{r}}$

$$
h_{\vec{r}}(t)=(2 \pi)^{-1} \int_{0}^{2 \pi} \mu(\vec{r}+t \vec{\eta}) d \varphi
$$

имеет ограниченную вариацию. При $\mu \in\left\{\mu^{\omega}, I\right\}$ функция $h_{\vec{r}}$ имеет компактный носитель, кусочно-дифференцируема, имеет не более конечного числа разрьвов первого рода. Точка $t_{0}=t_{0}(\vec{r})$ является точкой нарушения гладкости функции $h_{\vec{r}}$, если окружность радиуса $t_{0}$ с центром в точке $\vec{r}$ касается хотя бы одной из кривых $\partial \mathscr{D}_{i}$. Если среди этих кривых есть окружность с центром в $\vec{r}$, то функция $h_{\vec{r}}$ может иметь разрыв первого рода. Если такой кривой нет, то точка нарушения гладкости отвечает касанию конечного порядка и производная $h_{\vec{r}}^{(1)} \in L^{1}$, что и завершает доказательство.

Заметим, что по ходу доказательства теоремы 2.1 будет дано еще одно доказательство формулы обрашения для $\mu=\chi_{\mathscr{D}} \in\left\{\mu^{\omega}, I\right\}$ и, с формальной точки зрения, сама формула обращения нигде ниже не используется.

Теорема о проекции позволяет представить величину $\widetilde{\mu}^{A}(\vec{r})(1.9)$ в виде

$$
\widetilde{\mu}^{A}(\vec{r})=\frac{1}{4 \pi^{2}} \int_{0}^{2 \pi} d \varphi \int_{0}^{\infty} \lambda R^{A}(\lambda) \frac{1}{2}(\widehat{\Pi}(\lambda)+\widehat{\Pi}(-\lambda)) \widehat{\mu}(\lambda \vec{\eta}) e^{i \lambda\langle\vec{r}, \vec{\eta}\rangle} d \lambda,
$$

и, таким образом, величина $\widetilde{\mu}^{A}(\vec{r})$ получается введением регуляризатора $\frac{1}{2} R^{A}(\lambda) \times$ $(\widehat{\Pi}(\lambda)+\widehat{\Pi}(-\lambda))$ в формулу обращения преобразования Фурье. 
Переходим к анализу ошибок восстановления. Во введении были определены: $\Delta \mu^{A}(\vec{r})$ - ошибка восстановления (полная) $(1.22), \Delta \mu_{T}^{A}(\vec{r})$ - ошибка дискретизации (полная) (1.28), $\Delta \mu_{q}^{A}(\vec{r})$ - ошибка дискретизации по $q(1.27)$, и ошибка регуляризации $\Delta \mu_{R}^{A}(\vec{r})$ (1.26). Введем еще ошибку дискретизации по $\varphi-\Delta \mu_{\varphi}^{A}(\vec{r})$ и перекрестныи член $\Delta \mu_{L}^{A}(\vec{r})$. Пусть величина $\mu_{\varphi}^{A}(\vec{r})$ определяется равенством

$$
\mu_{\varphi}^{A}(\vec{r})=\frac{1}{2 \pi} \sum_{k=0}^{2 N_{\varphi}-1} \widetilde{\Psi}^{A}\left(\varphi_{k},\left\langle\vec{r}, \vec{\eta}_{k}\right\rangle\right) \Delta \varphi
$$

Тогда

$$
\Delta \mu_{\varphi}^{A}(\vec{r})=\mu_{\varphi}^{A}(\vec{r})-\widetilde{\mu}^{A}(\vec{r}), \quad \Delta \mu_{L}^{A}(\vec{r})=\widetilde{\Delta \mu_{\varphi}^{A}}(\vec{r})-\Delta \mu_{\varphi}^{A}(\vec{r})
$$

где величина $\widetilde{\Delta \mu}(\vec{\varphi})$ определена в (1.29). Теперь полную ошибку дискретизации можно представить в виде

$$
\Delta \mu_{T}^{A}(\vec{r})=\Delta \mu_{q}^{A}(\vec{r})+\Delta \mu_{\varphi}^{A}(\vec{r})+\Delta \mu_{L}^{A}(\vec{r})
$$

Начиная с этого места, будем считать, что

$$
\mu(\vec{r})=\chi_{\mathscr{D}}(\vec{r}), \quad \mu \in\left\{\mu^{\omega}, I\right\}
$$

Покажем, что в этом случае ошибка дискретизации $\Delta \mu_{T}^{A}(\vec{r})$ может быть представлена в виде двойного ряда трехкратных ОИ (1.32), и это представление согласуется с разложением (3.3).

Прежде всего отметим, что из определения класса $\{A\}$ рассматриваемых алгоритмов и приборных функций (см. (2.2)-(2.5)) и функций $u_{n}, v_{n}(1.35)$ следует оценка

$$
\begin{aligned}
& \left|\partial_{\varphi}^{p_{1}} \partial_{\xi}^{p-p_{1}} u_{n}\right| \leqslant C_{p} A(n),
\end{aligned}
$$

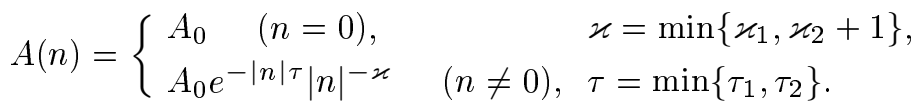

Определим функцию $E$ на $\mathbb{R}^{2}$ равенством

$$
E(\vec{z})=\frac{1}{2 N_{\varphi}} \sum_{k=0}^{2 N_{\varphi}-1} e^{i\left\langle\vec{z}, \vec{\eta}_{k}\right\rangle} \quad\left(\vec{z} \in \mathbb{R}^{2}, \vec{\eta}_{k}=\left(\cos \varphi_{k}, \sin \varphi_{k}\right)\right)
$$

и через $\vec{\nabla} E$ обозначим ее градиент

$$
(\vec{\nabla} E)(\vec{z})=\left(\frac{\partial E}{\partial z_{x}}, \frac{\partial E}{\partial z_{y}}\right)=\frac{i}{2 N_{\varphi}} \sum_{k=0}^{2 N_{\varphi}-1} \vec{\eta}_{k} e^{i\left\langle\vec{z}, \vec{\eta}_{k}\right\rangle}
$$

Основой дальнейших рассуждений служит 
Лемма 3.2. Пусть $\mu=\chi_{\mathscr{D}} \in\left\{\mu^{\omega}, I\right\}, A \in\{A\}$. Тогда имеет место равенство

$$
\begin{aligned}
& \mu^{A}\left(\vec{r}_{0}\right)=\Omega \sum_{n} e^{i 2 n \Omega \delta} \int_{0}^{\infty} v_{n}(\xi) d \xi \int_{\partial \mathscr{D}} d s[\vec{\tau}, \vec{\nabla} E(\vec{z})], \\
& \vec{z}=\Omega \xi \vec{l}(s)+2 n \Omega \vec{r}(s) \quad\left(\vec{l}(s)=\vec{r}(s)-\vec{r}_{0}, \vec{r}(s) \in \partial \mathscr{D}\right),
\end{aligned}
$$

где $\delta$ определено в (1.3).

ДокАЗАТЕЛЬСТво. По определению величины $\Psi^{A}\left(\varphi_{k}, a\right)(1.10)$

$$
\Psi^{A}\left(\varphi_{k}, a\right)=\sum_{j} f(j), \quad f(x)=H^{A}(a-\delta-x \Delta q) J^{\Pi}\left(\varphi_{k}, x \Delta q+\delta\right) \Delta q .
$$

Функция $f \in L^{1}$ и имеет ограниченную вариацию на всей оси. При этих условиях справедлива формула Пуассона [29]:

$$
\sum_{j} f(j)=\sum_{n} \int e^{i 2 \pi n x} f(x) d x
$$

и, следовательно,

$$
\Psi^{A}\left(\varphi_{k}, a\right)=\sum_{n} e^{i 2 n \Omega \delta} \int H^{A}(a-t) J^{\Pi}\left(\varphi_{k}, t\right) e^{-i 2 \pi n t} d t
$$

Используя теорему о проекции и теорему о свертке, получим, что для $\mu=g \chi_{\mathscr{D}} \in$ $\left\{\mu^{\omega}, I\right\}$ имеет место равенство

$$
\mu^{A}\left(\vec{r}_{0}\right)=\sum_{n} e^{i 2 n \Omega \delta} \int_{0}^{\infty} \widehat{H}^{A}(\lambda) \frac{1}{2}(\widehat{\Pi}(\lambda)+\widehat{\Pi}(-\lambda)) \iint_{\mathscr{D}} g(\vec{r}) E(\vec{z}) d x d y
$$

По формуле Грина при $g \equiv 1\left(\mu=\chi_{\mathscr{D}}\right)$

$$
\widehat{\mu}(\lambda \vec{\eta})=\iint_{\mathscr{D}} e^{i \lambda\langle\vec{r}, \vec{\eta}\rangle} d x d y=\frac{i}{\lambda} \int_{\partial \mathscr{D}} e^{i \lambda\langle\vec{r}(s), \vec{\eta}\rangle}[\vec{\tau}, \vec{\eta}] d s
$$

и, следовательно,

$$
\iint E(\vec{z}) d x d y=\frac{1}{\lambda+2 n \Omega} \int_{\partial \mathscr{D}}[\vec{\tau}, \vec{\nabla} E(\vec{z})] d s .
$$

Остается воспользоваться условием однородности (1.13), чтобы из (3.7) получить нужньй результат (3.6).

Лемма 3.2 позволяет в случае $\mu=\chi_{\mathscr{D}}$ дать точную формулу, представляюшую $\mu^{A}\left(\vec{r}_{0}\right)(1.10)$ в виде ряда ОИ с $\Omega$ в качестве большого параметра. 
Tеорема 3.2. Пусть $\mu=\chi_{\mathscr{D}} \in\left\{\mu^{\omega}, I\right\}, A \in\{A\}$ и, кроме того,

$$
\xi^{2} v_{n} \in L^{1} \quad\left(\int_{0}^{\infty} \xi^{2}\left|v_{n}(\xi)\right| d \xi<\infty\right) .
$$

Тогда имеет место представление (1.32) и при этом

$$
\begin{array}{cc}
\Delta \mu_{T}^{A}\left(\vec{r}_{0}\right)=\frac{i \Omega}{2 \pi} \sum_{n, m}^{\prime} e^{i 2 n \Omega \delta} J_{n m}, & \Delta \mu_{q}^{A}\left(\vec{r}_{0}\right)=\frac{i \Omega}{2 \pi} \sum_{n \neq 0} J_{n 0} e^{i 2 n \Omega \delta}, \\
\Delta \mu_{\varphi}^{A}\left(\vec{r}_{0}\right)=\frac{i \Omega}{2 \pi} \sum_{m \neq 0} J_{0 m}, & \Delta \mu_{L}^{A}\left(\vec{r}_{0}\right)=\frac{i \Omega}{2 \pi} \sum_{\substack{n \neq 0 \\
m \neq 0}} J_{n m} e^{i 2 n \Omega \delta}, \\
\widetilde{\mu}^{A}\left(\vec{r}_{0}\right) \equiv \mu_{R}^{A}\left(\vec{r}_{0}\right)=\frac{i \Omega}{2 \pi} J_{00},
\end{array}
$$

а $\delta \quad(0 \leqslant \delta<\Delta q)$ определено в (1.3).

ДокАЗАТЕЛЬСТво. Воспользуемся формулой Пуассона на окружности

$$
\sum_{k=0}^{2 N_{\varphi}-1} f\left(\varphi_{k}\right) \Delta \varphi=\sum_{m} \int_{0}^{2 \pi} e^{i 2 N_{\varphi} m \varphi} f(\varphi) d \varphi .
$$

Для справедливости этой формулы достаточно сходимости ряда Фурье функции $f$ в точках $\varphi_{k}$, т.е. ограниченности вариации этой функции в окрестностях точек $\varphi_{k}$. С помощью (3.13) запишем величину $\vec{\nabla} E(\vec{z})(3.5)$ в виде

$$
\vec{\nabla} E(\vec{z})=\frac{i}{2 \pi} \sum_{m} \int_{0}^{2 \pi} \vec{\eta} e^{-i 2 N_{\varphi} m \varphi+i\langle\vec{z}, \vec{\eta}\rangle} d \varphi .
$$

Подставляя это выражение в (3.6), получим

$$
\mu^{A}\left(\vec{r}_{0}\right)=\frac{i \Omega}{2 \pi} \sum_{n} e^{i 2 n \Omega \delta} \int_{0}^{\infty} v_{n}(\xi) d \xi \sum_{m} \int_{0}^{2 \pi} d \varphi \int_{\partial \mathscr{D}} d s e^{i \Omega \Phi_{n m}}[\vec{\tau}, \vec{\eta}] .
$$

Остается показать, что в правой части этого равенства можно изменить порядок суммирования по $m$ и интегрирования по $\xi$. Обозначим через $I_{m}$ интеграл по $\varphi, s$ в правой части (3.15):

$$
I_{m}=\int_{\partial \mathscr{D}} d s \int_{0}^{2 \pi} e^{i 2 N_{\varphi} m \varphi} h(\varphi) d \varphi, \quad h(\varphi)=[\vec{\tau}, \vec{\eta}] e^{i \Omega\langle\vec{\xi}(s)+2 n \vec{r}(s), \vec{\eta}\rangle} .
$$

Интегрируя два раза по частям, получим $I_{m}=O\left(\xi^{2} m^{-2}\right)$, и тогда из условия (3.9) следует сходимость интеграла $\int_{0}^{\infty}\left|v_{n}(\xi)\right| I_{m} \mid d \xi$. По принципу абсолютной сходимости этого достаточно для справедливости (1.32). Равенства (3.10), (3.11) доказываются точно так же. Подставляя в (3.2) выражение $(3.8)$ для $\widehat{\mu}(\lambda \vec{\eta})$, получим (3.12). 
Теорема 3.2 фактически ниже не используется. Будем исходить непосредственно из леммы 3.2 и покажем, что ряд (3.10) для $\Delta \mu_{T}^{A}\left(\vec{r}_{0}\right)$ можно заменить конечной суммой. Тем самым будет дано новое доказательство теоремы 3.2. В качестве первого шага перейдем от ряда $\vec{\nabla} E(\vec{z})(3.14)$ к конечной сумме

$$
\vec{\nabla}_{M} E(\vec{z})=\frac{i}{2 \pi} \sum_{|m|<M} \int_{0}^{2 \pi} e^{-i 2 N_{\varphi} m \varphi+i\langle\vec{z}, \vec{\eta}\rangle} d \varphi .
$$

Для оценки величины $\vec{\nabla} E(\vec{z})-\vec{\nabla}_{M} E(\vec{z})$ введем параметр

$$
\beta=\frac{|\vec{z}|}{2 N_{\varphi} M-1} \quad(M \geqslant 1)
$$

и функщию

$$
f(\beta)=\ln \frac{1+\sqrt{1-\beta^{2}}}{\beta}-\sqrt{1-\beta^{2}},
$$

определенную при $0<\beta<1$.

Лемма 3.3. Пусть $M \geqslant 1$ и випполняются условия

$$
0<\beta \equiv \frac{|\vec{z}|}{2 N_{\varphi} M-1}<1, \quad 2 N_{\varphi} f(\beta) \geqslant 1 .
$$

Тогда

$$
\left|\vec{\nabla} E(\vec{z})-\vec{\nabla}_{M} E(\vec{z})\right| \leqslant \frac{C N_{\varphi}(M+2) e^{-\left(2 N_{\varphi} M-1\right) f(\beta)}}{\left(1-\beta^{2}\right)^{1 / 4} \sqrt{2 N_{\varphi} M-1}}
$$

и в качестве константы $С$ можно взять $8 \pi^{-1 / 2}$.

ДокАЗАТЕЛЬСТво. Воспользуемся интегральньм представлением для функций Бесселя [30]

$$
J_{2 N_{\varphi} m}(|\vec{z}|)=(-1)^{N_{\varphi} m} e^{i 2 N_{\varphi} m \theta} \int_{0}^{2 \pi} e^{-i 2 N_{\varphi} m \varphi+i\langle\vec{z}, \vec{\eta}\rangle} d \varphi \quad(\vec{z}=|\vec{z}|(\cos \theta, \sin \theta)) .
$$

Пусть $\vec{\nabla} E(\vec{z})-\vec{\nabla}_{M} E(\vec{z})=\left(\Delta_{x}^{M} E, \Delta_{y}^{M} E\right)$. Тогда

$$
\left|\Delta_{x}^{M} E(\vec{z})\right| \leqslant \sum_{|m| \geqslant M}\left|\frac{\partial}{\partial z_{x}}\left(e^{i 2 N_{\varphi} m \theta} J_{2 N_{\varphi} m}(|\vec{z}|)\right)\right| .
$$

Так как $\left|\frac{\partial \theta}{\partial z_{x}}\right| \leqslant|\vec{z}|^{-1},\left|\frac{\partial(|\vec{z}|)}{\partial z_{x}}\right| \leqslant 1$, то, используя соотношения

$$
J_{-n}(x)=(-1)^{n} J_{n}(x), \quad J_{n}^{(1)}(x)=\frac{1}{2}\left(J_{n-1}(x)-J_{n+1}(x)\right),
$$


получим, что

$$
\left|\Delta_{x}^{M} E(\vec{z})\right| \leqslant \sum_{|m| \geqslant M}\left(\frac{4 N_{\varphi}|m|}{|\vec{z}|} J_{2 N_{\varphi} m}(|\vec{z}|)+J_{2 N_{\varphi} m-1}(|\vec{z}|)+J_{2 N_{\varphi} m+1}(|\vec{z}|)\right)
$$

Для оценки входяших в правую часть этого неравенства функций Бесселя воспользуемся формулой Карлини [30], согласно которой

$$
\left|J_{\nu}(\nu x)\right| \leqslant G(\nu, x), \quad G(\nu, x)=\frac{e^{-\nu f(x)}}{\left(1-x^{2}\right)^{1 / 4} \sqrt{2 \pi \nu}} \quad(0<x<1),
$$

где $f$ - функция, определенная в (3.17). Так как $f^{(1)}(x)=-\frac{\sqrt{1-x^{2}}}{x}<0$, то при условии (3.18) из (3.20) и (3.21) следует, что

$$
\begin{aligned}
\left|\Delta_{x}^{M} E(\vec{z})\right| & \leqslant 8 \sum_{m \geqslant M} \frac{N_{\varphi} m}{|\vec{z}|} G\left(2 N_{\varphi} m-1, \frac{|\vec{z}|}{2 N_{\varphi} m-1}\right) \\
& \leqslant \frac{8 N_{\varphi}}{|\vec{z}|} \frac{\exp (f(\beta))}{\left(1-\beta^{2}\right)^{1 / 4} \sqrt{2 \pi\left(2 N_{\varphi} M-1\right)}} \sum_{m \geqslant M} m \exp \left(-2 N_{\varphi} m f(\beta)\right) .
\end{aligned}
$$

В силу условия (3.18) это ряд с монотонно убьваюшими членами и, следовательно,

$$
\sum_{m \geqslant M} m e^{-2 N_{\varphi} m f(\beta)} \leqslant(M+2) e^{-2 N_{\varphi} M f(\beta)} .
$$

Теперь из (3.22) получаем

$$
\left|\nabla_{x}^{M} E(\vec{z})\right| \leqslant \frac{8 N_{\varphi}(M+2) e^{-\left(2 N_{\varphi} M-1\right) f(\beta)}}{|\vec{z}|\left(1-\beta^{2}\right)^{1 / 4} \sqrt{2 \pi\left(2 N_{\varphi} M-1\right)}}
$$

Эта же оценка справедлива и для $\left|\nabla_{y}^{M} E(\vec{z})\right|$. Отсюда сразу следует нужньй результат (3.19).

При $\beta \leqslant \frac{1}{2}$ верно неравенство $f(\beta) \geqslant 0,45$, и, следовательно, если

$$
N_{\varphi} \geqslant 2, \quad M \geqslant 1, \quad \frac{|\vec{z}|}{2 N_{\varphi} M-1} \leqslant 0.5
$$

то имеет место оценка

$$
\left|\vec{\nabla} E(\vec{z})-\vec{\nabla}_{M} E(\vec{z})\right| \leqslant C e^{-0.9 N_{\varphi} M} \quad\left(C=\frac{24 e^{2} \sqrt{2}}{\sqrt{\pi} 3^{1 / 4}}\right),
$$

которой мы и будем пользоваться ниже.

Ошибка регуляризации полностью исследована в работе [25], и простейшая нужная нам оценка этой величины приведена в приложении. 
Рассмотрим ошибку дискретизации $\Delta \mu_{T}^{A}\left(\vec{r}_{0}\right)(1.28)$, которая уже была представлена в виде ряда ОИ (3.10). Чтобы вместо этого ряда получить конечную сумму интегралов по конечной области, введем величину

$$
\Delta \mu_{T F}^{A}\left(\vec{r}_{0}\right)=\frac{i \Omega}{2 \pi} \sum_{n, m}^{F} \int_{0}^{B} d \xi \int_{0}^{2 \pi} d \varphi \int_{\partial \mathscr{D}} d s e^{i \Omega \Phi_{n m}} u_{n},
$$

где по определению

$$
\sum_{n, m}^{F} a_{n m}=\sum_{|n| \leqslant N_{1}} \sum_{\substack{|m|<M \\(n, m) \neq(0,0)}} a_{n m} .
$$

Оценим ошибку обрезания $\Delta_{F} \mu^{A}\left(\vec{r}_{0}\right)$, определяемую равенством

$$
\Delta \mu_{T}^{A}\left(\vec{r}_{0}\right)=\Delta \mu_{T F}^{A}\left(\vec{r}_{0}\right)+\Delta_{F} \mu^{A}\left(\vec{r}_{0}\right) .
$$

Лемма 3.4. Пусть $\mu=\chi_{\mathscr{D}} \in\left\{\mu^{\omega}, I\right\}, A \in\{A\}$ и выполняются условия

$$
M \geqslant \frac{1}{2 N_{\varphi}}+\frac{B+N_{1}}{L}, \quad N_{\varphi} \geqslant 2, \quad N_{1} \geqslant B, \quad \varkappa_{1}>1, \quad \varkappa_{2}>0 .
$$

Тогда для ошибки обрезания $\Delta_{F} \mu^{A}\left(\vec{r}_{0}\right)$ в (3.26) справедлива оченка

$$
\left|\Delta_{F} \mu^{A}\left(\vec{r}_{0}\right)\right| \leqslant \mathscr{L} \Omega\left[C_{1} \frac{e^{-\tau_{1} B}}{B^{\varkappa_{1}-1}}+C_{2} \frac{e^{-\tau_{2} N_{1}}}{N_{1}^{\varkappa_{2}}}+C_{3} e^{-0.9 N_{\varphi} M}+C_{4} \frac{e^{-\left(\tau_{1}+\tau_{2}\right) B}}{B^{\varkappa_{1}+\varkappa_{2}-1}}\right] .
$$

Явный вид констант $C_{k}=C_{k}\left(\vec{A}, \varkappa_{i}, \tau_{i}\right)$ указан ниже и

$$
C_{k}=C_{k}\left(\vec{A}, \varkappa_{i}, \tau_{i}\right) \leqslant C A_{0}\left(\frac{1}{\varkappa_{1}-1}+\frac{1}{\varkappa_{2}}+\frac{1}{\varkappa_{2}\left(\varkappa_{1}-1\right)}\right),
$$

где величина $A_{0}$ определена в (2.6).

ДокАЗАТЕЛЬСтво. В качестве первого шага перейдем к конечному верхнему пределу интегрирования по $\xi$. Будем исходить из представления (3.6) и введем величины $\mu_{B}^{A}\left(\vec{r}_{0}\right), \Delta \mu_{B}^{A}\left(\vec{r}_{0}\right):$

$$
\begin{gathered}
\mu^{A}\left(\vec{r}_{0}\right)=\mu_{B}^{A}\left(\vec{r}_{0}\right)+\Delta \mu_{B}^{A}\left(\vec{r}_{0}\right), \\
\mu_{B}^{A}\left(\vec{r}_{0}\right)=\Omega \sum_{n} e^{i 2 n \Omega \delta} \int_{0}^{B} v_{n}(\xi) d \xi \int_{\partial \mathscr{D}} d s[\vec{\tau}, \vec{\nabla} E(\vec{z})] .
\end{gathered}
$$

Используя тривиальные оценки $\left|\nabla_{x} E(\vec{z})\right| \leqslant 1,\left|\nabla_{y} E(\vec{z})\right| \leqslant 1$, согласно определению алгоритма $A \in\{A\}(2.2)-(2.5)$ получим, что

$$
\begin{gathered}
\left|\Delta \mu_{B}^{A}\left(\vec{r}_{0}\right)\right| \leqslant 2 \Omega \mathscr{L} \int_{B}^{\infty}\left|P_{A}(\xi)\right| d \xi \cdot \max _{\xi \geqslant B} w(\xi), \\
w(\xi)=\sum_{n} \frac{\left|T_{A}(\xi)\right|\left|S_{n}^{\Pi}(\xi+2 n)\right|}{|\xi+2 n|} .
\end{gathered}
$$


Функция $w$ при $A \in\{A\}$ легко оценивается:

$$
w(\xi) \leqslant 3 A_{2} A_{4}\left(1+\varkappa_{2}^{-1}\right)+A_{1} A_{5},
$$

и, следовательно,

$$
\left|\Delta \mu_{B}^{A}\left(\vec{r}_{0}\right)\right| \leqslant C_{1} \mathscr{L} \Omega \frac{e^{-\tau_{1} B}}{B^{\varkappa_{1}}-1}, \quad C_{1}=\frac{2 A_{0}}{\varkappa_{1}-1}\left[\frac{3 A_{2} A_{4}}{1+\varkappa_{2}^{-1}}+A_{1} A_{5}\right] .
$$

Второй шаг состоит в переходе к конечной сумме по $n$ в $\mu_{B}^{A}\left(\vec{r}_{0}\right)(3.30)$ :

$$
\mu_{B}^{A}\left(\vec{r}_{0}\right)=\mu_{B N_{1}}^{A}\left(\vec{r}_{0}\right)+\Delta \mu_{B N_{1}}^{A}\left(\vec{r}_{0}\right)
$$

где по определению

$$
\mu_{B N_{1}}^{A}\left(\vec{r}_{0}\right)=\Omega \sum_{|n| \leqslant N_{1}} e^{i 2 n \Omega \delta} \int_{0}^{B} v_{n}(\xi) d \xi \int_{\partial \mathscr{D}} d s[\vec{\tau}, \vec{\nabla} E(\vec{z})]
$$

и, так как $|\xi+2 n| \geqslant|n|$ при $N \geqslant B_{1}$, то

$$
\begin{aligned}
\left|\Delta \mu_{B N_{1}}^{A}\left(\vec{r}_{0}\right)\right| & \leqslant 2 \Omega \mathscr{L} \sum_{|n| \geqslant N_{1}+1} \int_{0}^{B} \frac{\left|S^{A}(\xi)\right|\left|S_{n}^{\Pi}(\xi+2 n)\right|}{|\xi+2 n|} d \xi \\
& \leqslant 2 \Omega \mathscr{L} A_{2} e^{-\tau_{2}\left(N_{1}+1\right)}\left\|S^{A}\right\|_{L^{1}} \varkappa_{2}^{-1} N_{1}^{-\varkappa 2} .
\end{aligned}
$$

Остается заметить, что при $A \in\{A\}$

$$
\left\|S^{A}\right\|_{L^{1}} \leqslant A_{4} \int_{0}^{\infty}\left|P_{A}(\xi)\right| d \xi \leqslant A_{4}\left(A_{3}+\frac{A_{6} e^{-\tau_{1}}}{\varkappa_{1}-1}\right)
$$

чтобы получить

$$
\left|\Delta \mu_{B N_{1}}^{A}\left(\vec{r}_{0}\right)\right| \leqslant C_{2} \frac{\Omega \mathscr{L} e^{-\alpha_{2} N_{1}}}{N_{1}^{\varkappa 2}}, \quad C_{2}=\frac{2 A_{2} e^{-\tau_{2}}}{\varkappa_{2}}\left(A_{3}+\frac{A_{0} e^{-\tau_{1}}}{\varkappa_{1}-1}\right) A_{4} .
$$

На третьем этапе переходим в $\Delta \mu_{B N_{1}}^{A}\left(\vec{r}_{0}\right)$ к конечной сумме по $m$ :

$$
\mu_{B N_{1}}^{A}\left(\vec{r}_{0}\right)=\mu_{B N_{1} M}^{A}\left(\vec{r}_{0}\right)+\Delta \mu_{B N_{1} M}^{A}\left(\vec{r}_{0}\right)
$$

где по определению

$$
\mu_{B N_{1} M}^{A}\left(\vec{r}_{0}\right)=\Omega \sum_{|n| \leqslant N_{1}} e^{i 2 n \Omega \delta} \int_{0}^{B} v_{n}(\xi) d \xi \int\left[\vec{\tau}, \vec{\nabla}_{M} E(\vec{z})\right] d s
$$

и величина $\vec{\nabla}_{M} E(\vec{z})$ определяется равенством $(3.16)$ при $\vec{z}=\Omega(\xi \vec{l}+2 n \vec{r})$. 
Оценим величину

$$
\Delta \mu_{B N_{1} M}^{A}\left(\vec{r}_{0}\right)=\Omega \sum_{|n| \leqslant N_{1}} e^{i 2 n \Omega \delta} \int_{0}^{B} v_{n}(\xi) d \xi \int_{\partial \mathscr{D}} d s\left[\vec{\tau}, \vec{\nabla} E(\vec{z})-\vec{\nabla}_{M} E(\vec{z})\right]
$$

Так как $|\vec{l}| \leqslant 1,|\vec{r}| \leqslant 0.5$, то $|z| \leqslant \Omega\left(B+N_{1}\right)$, вьполняется условие (3.23), и, используя (3.24), получим оценку

$$
\begin{gathered}
\left|\Delta \mu_{B N_{1} M}^{A}\left(\vec{r}_{0}\right)\right| \leqslant C \Omega \mathscr{L} e^{-0.9 N_{\varphi} M} \sum_{|n| \leqslant N_{1}} \int_{0}^{B}\left|v_{n}(\xi)\right| d \xi \leqslant C_{3} \Omega \mathscr{L} e^{-0.9 N_{\varphi} M} \\
C_{3}=C\left(A_{3}+A_{6} e^{-\tau_{2}}\left(\varkappa_{1}-1\right)^{-1}\right)\left(3 A_{2} A_{4}\left(1+\varkappa_{2}^{-1}\right)+A_{1} A_{5}\right)
\end{gathered}
$$

что и дает третий член в правой части (3.28). Наличие в ней четвертого члена связано с тем, что в выражении $(3.22)$ для величины $\mu_{R}^{A}\left(\vec{r}_{0}\right)$ интегрирование по $\xi$ ведется от 0 до $\infty$, а в $\mu_{B N_{1} M}^{A}\left(\vec{r}_{0}\right)$ и в члене с $n=0, m=0$ интегрирование по $\xi$ ведется от 0 до $B$. Величина

$$
\Delta \mu_{0 B}\left(\vec{r}_{0}\right)=\frac{i \Omega}{2 \pi} \int_{B}^{\infty} d \xi \int_{0}^{2 \pi} d \varphi \int_{\partial \mathscr{D}} d s e^{i \Omega \Phi_{00}} u_{0}
$$

легко оценивается:

$$
\left|\Delta \mu_{0 B}^{A}\left(\vec{r}_{0}\right)\right| \leqslant \frac{C_{4} \mathscr{L} \Omega e^{-\left(\tau_{1}+\tau_{2}\right) B}}{B^{\varkappa_{2}+\varkappa_{1}-1}}, \quad C_{4}=\frac{A_{2} A_{5} A_{6}}{\varkappa_{1}+\varkappa_{2}-1}
$$

и дает четвертый член в правой части (3.28). Подставляя в (3.36) выражение $\vec{\nabla}_{M} E(\vec{z})$ из (3.16) при $\vec{z}=\Omega(\xi \vec{l}+2 n \vec{r})$, получим

$$
\mu_{B N_{1} M}^{A}=\mu_{R}^{A}\left(\vec{r}_{0}\right)-\Delta \mu_{0 B}^{A}\left(\vec{r}_{0}\right)+\Delta \mu_{T F}^{A}\left(\vec{r}_{0}\right) .
$$

Это равенство вместе с (3.32), (3.35) после подстановки их в (3.30) приводит к равенству (3.26), в котором

$$
\Delta_{F} \mu^{A}\left(\vec{r}_{0}\right)=\Delta \mu_{B}^{A}\left(\vec{r}_{0}\right)+\Delta \mu_{B N_{1}}^{A}\left(\vec{r}_{0}\right)+\Delta \mu_{B N_{1} M}^{A}\left(\vec{r}_{0}\right)-\Delta \mu_{0 B}^{A}\left(\vec{r}_{0}\right) .
$$

Это вместе с полученными оценками величин в правой части и дает нужный результат (3.28).

Заметим, что оценка (3.28) равномерна по $\tau_{1}, \tau_{2}$ и в ней можно положить $\tau_{1}=\tau_{2}=0$.

Рассмотрим, как изменятся оценки ошибки обрезания $\Delta_{F} \mu^{A}\left(\vec{r}_{0}\right)$ при дополнительных предположениях об алгоритме $A$. Соответствуюшие утверждения сформулируем в виде дополнения в лемме 3.4. Это дополнение доказьвается так же, как и сама лемма 3.4 . 


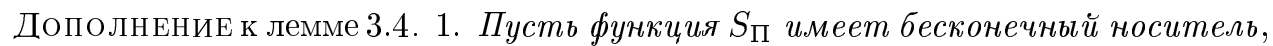
а функция $S^{A}-$ конечныц й, и пусть $S^{A}(\xi)=0 \quad\left(|\xi| \geqslant a_{A}\right)$. Тогда в оценке (3.28) можно положить $B=a_{A}, C_{1}=C_{4}=0$.

2. Пусть функиия $S^{A}$ имеет бесконечный носитель, а функиия $S_{\Pi}-$ конечный, и пусть $S_{\Pi}(\xi)=0 \quad\left(|\xi| \geqslant a_{\Pi}\right)$. Тогда в оценке $(3.28)$ можно положить $C_{2}=$ $C_{4}=0$ и в выражениях $C_{1}, C_{2}$ сделать замену $A_{2} \rightarrow a_{\Pi} A_{1}, \varkappa_{2} \rightarrow \infty$.

3. Пусть обе функиии $S^{A}, S_{\Pi}$ имеют конечный носитель и

$$
S^{A}(\xi)=0 \quad\left(|\xi| \geqslant a_{A}\right), \quad S_{\Pi}(\xi)=0 \quad\left(|\xi| \geqslant a_{\Pi}\right) .
$$

Тогда можно положить $B=a_{A}$, а условие $N_{1} \geqslant B$ в (3.27) заменить на $N_{1} \geqslant 0.5\left(a_{A}+a_{\Pi}\right)$. В оченке (3.28) можно положить $C_{1}=C_{2}=C_{4}=0$, а в виражсении для $C_{3}$ сделать замену $A_{2} \rightarrow a_{\Pi} A_{1}, \varkappa_{1}\left(\varkappa_{2}\right) \rightarrow \infty$. Таким образом, в этом случае ошибка обрезания экспоненииально мала.

Если вьполняются условия (3.42) и сумма $a_{A}+a_{\Pi}$ достаточно мала, то экспоненшиально малы все ошибки дискретизации.

Лемма 3.5. Пусть $\mu=\chi_{\mathscr{D}} \in\left\{\mu^{\omega}, I\right\}, A \in\{A\}$, выполнены условия (3.42) $и$ при этом

$$
a_{\Pi}+a_{A}<2, \quad\left(2 N_{\varphi}\right)^{-1}+a_{A} L^{-1}<1 .
$$

Тогда имеет место оченка

$$
\left|\Delta \mu_{T}^{A}\left(\vec{r}_{0}\right)\right|=\left|\Delta_{F} \mu^{A}\left(\vec{r}_{0}\right)\right| \leqslant C_{3} \Omega \mathscr{L} e^{-0.9 N_{\varphi}} .
$$

ДокАЗАТЕЛЬСТВо. При $a_{\Pi}+a_{A}<2 u_{n} \equiv 0(|n| \neq 0)$ и условие (3.27) вьПолняется при $M=1$. В этом случае $\Delta \mu_{T F}^{A}(\vec{r})=0$ и остается воспользоваться утверждением 3 из дополнения к лемме 3.4 .

Заметим, что лемма 3.5 верна для любого $\mu \in\left\{\mu^{\omega}, I\right\}$. Эта лемма вместе с оценками ошибки регуляризации (см. приложение) показьвает, что существуют сходяшиеся алгоритмы высокого разрешения и на классе $\left\{\mu^{\omega}, I\right\}$ показатель сходимости может быть сколь угодно большим. К сожалению, на практике условия $a_{\Pi}+a_{A}<2$ никогда не выполняются.

\section{§4. Анализ критических точек}

В $\S 3$ было показано, что оценка ошибки дискретизации сводится к исследованию трехкратных ОИ $J_{n m}(1.33)$ с фазой

$$
\begin{gathered}
\Phi(z) \equiv \Phi(\varphi, \xi, s)=-2 L m \varphi+\langle\xi \vec{l}(s)+2 n \vec{r}(s), \vec{\eta}\rangle \\
\left(\vec{l}(s)=\vec{r}(s)-\vec{r}_{0}, \quad \vec{r}(s) \in \partial \mathscr{D}, \quad z=\left(z_{1}, z_{2}, z_{3}\right) \equiv(\varphi, \xi, s)\right) .
\end{gathered}
$$

В этом параграфе будет дан анализ критических точек $z_{c}=\left(\varphi_{c}, \xi_{c}, s_{c}\right)$ фазы $\Phi$ при $(n, m) \neq(0,0)$. Везде ниже предполагается, что $d\left(\vec{r}_{0}, \partial \mathscr{D}\right) \geqslant d_{0}>0$. Целочисленные индексы $n, m$ считаются фиксированными, и зависимости различных величин от $n, m, \vec{r}_{0}$ часто явно не указывваются. 
Покажем, что критические точки $z_{c}$ при $m \neq 0$ или $\vec{r}_{0} \neq 0$ изолированы, имеют corank $\leqslant 1$ и, следовательно, все особенности фазы $\Phi$ принадлежат к классам $A_{k}$ [23].

Рассмотрим критические точки $\varphi_{c}(s), \xi_{c}(s)$ фазы $\Phi$ как функции двух переменных $\varphi, \xi$, считая $s$ параметром. Эти точки задаются выбором знака $\varepsilon_{ \pm}= \pm 1$, $\left(\varphi_{c}(s), \xi_{c}(s)\right)=\left(\varphi_{ \pm}(s), \xi_{ \pm}(s)\right)$ и определяются из соотношений

$$
\begin{gathered}
\vec{\eta}_{ \pm}(s)=\varepsilon_{ \pm} l_{1}^{*}(s), \quad \varphi_{-}(s)=\varphi_{+}(s)+\pi, \quad\left\langle\vec{l}, \vec{\eta}_{ \pm}\right\rangle=0, \\
\xi_{ \pm}(s) \equiv \xi_{n m}^{ \pm}(s)=-\frac{2 L m+2 n\left[\vec{r}, \vec{\eta}_{ \pm}\right]}{\left[\vec{l}, \vec{\eta}_{ \pm}\right]}=-\varepsilon_{ \pm} l^{-1}\left(2 L m+2 n\left[\vec{r}, \vec{\eta}_{ \pm}\right]\right),
\end{gathered}
$$

где $\vec{l}_{1}^{*}$ - единичный вектор, перпендикулярный к $\vec{l}=l \cdot \vec{l}_{1}\left(\left[\overrightarrow{l_{1}}, \vec{l}_{1}^{*}\right]=1, l_{1}^{*}=l^{-1}\left(-l_{y}, l_{x}\right)\right.$, $\left.\left\langle\vec{l}, \vec{l}_{1}^{*}\right\rangle=0\right)$. Из (4.2) следует, что

$$
\xi_{ \pm}(s)+2 n=-\frac{2 L m+2 n\left[\vec{r}_{0}, \vec{\eta}_{ \pm}\right]}{\left[\vec{l}, \vec{\eta}_{ \pm}\right]} .
$$

Матрица $\Phi_{c}^{\prime \prime}=\Phi_{ \pm}^{\prime \prime}$ вторых производных легко вычисляется:

$$
\begin{gathered}
\Phi_{ \pm}^{\prime \prime}=\left(\begin{array}{cc}
a_{11} & a_{12} \\
a_{12} & 0
\end{array}\right), \quad a_{i k}=\frac{\partial^{2} \Phi}{\partial z_{i} \partial z_{k}}\left(\varphi_{T}, \xi_{ \pm}\right) \quad(i, k=1,2), \\
a_{11}=-2 n\left\langle\vec{r}_{0}, \vec{\eta}_{ \pm}\right\rangle, \quad a_{12}=-\varepsilon_{ \pm} l, \quad \operatorname{det} \Phi_{ \pm}^{\prime \prime}=-l^{2} .
\end{gathered}
$$

Таким образом, критические точки $\left(\varphi_{ \pm}, \xi_{ \pm}\right)$при $d\left(\vec{r}_{0}, \partial \mathscr{D}\right)>0$ невырождены, и остается рассмотреть критические точки фазы

$$
S(s) \equiv S_{ \pm}(s)=\Phi\left(\varphi_{ \pm}(s), \xi_{ \pm}(s), s\right) .
$$

Считая выбор знака $\varepsilon_{ \pm}$фиксированным, ниже мы его часто не указываем, $\varphi(s) \equiv$ $\varphi_{ \pm}(s), \xi(s) \equiv \xi_{ \pm}(s)$. Так как $\left\langle\vec{l}, \vec{\eta}_{ \pm}\right\rangle=0$, то $\left\langle\vec{r}, \vec{\eta}_{ \pm}\right\rangle=\left\langle\vec{r}_{0}, \vec{\eta}_{ \pm}\right\rangle$, и, следовательно, функция $S \equiv S_{ \pm}$задается соотношениями

$$
\begin{aligned}
S(s) & =-2 L m \varphi(s)+2 n\left\langle\vec{r}_{0}, \vec{\eta}(s)\right\rangle, \\
\langle\vec{l}(s), \vec{\eta}(s)\rangle & =0 \quad\left(\vec{l}(s) \equiv \vec{l}\left(r_{0}, s\right)=\vec{r}(s)-\vec{r}_{0}\right) .
\end{aligned}
$$

Везде ниже в этом параграфе предполагается, что

$$
m \neq 0 \text { либо } \vec{r}_{0} \neq 0 .
$$

Найдем критические точки $s_{c}$ фазы $S$ и покажем, что они изолированы и имеют конечную кратность. Дифференщируя равенство $\langle\vec{l}, \vec{\eta}\rangle=0$, определяющее зависимость $\varphi(s)$, имеем

$$
\varphi^{(1)}(s)=l^{-2}[\vec{l}, \vec{\tau}],
$$

и, следовательно,

$$
S^{(1)}(s)=-\left(2 L m+2 n\left[\vec{r}_{0}, \vec{\eta}(s)\right]\right) \varphi^{(1)}(s)=(\xi(s)+2 n) \varepsilon_{ \pm} \frac{[\vec{l}, \vec{\tau}]}{l} .
$$


Таким образом, критические точки $s_{c}$ могут быть одного из следующих трех muпов:

$$
\begin{array}{llll}
\text { 1-й тип } & \left(s_{c} \equiv s_{c}^{1}\right): & {\left[\vec{l}\left(s_{c}^{1}\right), \vec{\tau}\left(s_{c}^{1}\right)\right]=0,} & \xi\left(s_{c}^{1}\right)+2 n \neq 0, \\
\text { 2-й тип } & \left(s_{c} \equiv s_{c}^{2}\right): & {\left[\vec{l}\left(s_{c}^{2}\right), \vec{\tau}\left(s_{c}^{2}\right)\right] \neq 0,} & \xi\left(s_{c}^{2}\right)+2 n=0, \\
\text { 3-й тип } & \left(s_{c} \equiv s_{c}^{3}\right): & {\left[\vec{l}\left(s_{c}^{3}\right), \vec{\tau}\left(s_{c}^{3}\right)\right]=0,} & \xi\left(s_{c}^{3}\right)+2 n=0 .
\end{array}
$$

Соответственно будем различать и три типа $z_{c}^{i}$ критических точек фазы $\Phi: z_{c}^{i}=$ $\left(\varphi_{c}^{i}, \xi_{c}^{i}, s_{c}^{i}\right)$. Здесь и ниже используются обозначения $\varphi_{c}^{i}=\varphi_{ \pm}\left(s_{c}^{i}\right), \xi_{c}^{i}=\xi_{ \pm}\left(s_{c}^{i}\right)$ $(i=1,2,3)$.

Рассмотрим критические точки первого типа. В этом случае

$$
\vec{l}\left(s_{c}^{1}\right)=\varepsilon_{1}^{c} \vec{\tau}\left(s_{c}^{1}\right) l\left(s_{c}^{1}\right), \quad \varepsilon_{1}^{c}= \pm 1,
$$

и для определения $s_{c}^{1} \equiv s_{c}^{1}\left(\vec{r}_{0}\right)$ достаточно из точки $\vec{r}_{0}$ провести касательные к $\partial \mathscr{D}$, $\vec{r}\left(s_{c}^{1}\right)$ - точки касания. Знак $\varepsilon_{1}^{c}$ при этом фиксируется автоматически и

$$
\vec{\eta}\left(s_{c}^{1}\right)=\varepsilon_{2}^{c} \vec{\nu}\left(s_{c}^{1}\right), \quad \varepsilon_{2}^{c}= \pm 1 .
$$

Знак $\varepsilon_{2}^{c}$ определяется выбором $\varepsilon_{ \pm}$

$$
\varepsilon_{1}^{c} \varepsilon_{2}^{c} \varepsilon_{ \pm}=-1
$$

Таким образом, для критических точек первого типа $z_{c}^{1}=\left(\varphi_{c}^{1}, \xi_{c}^{1}, s_{c}^{1}\right)$ величина $s_{c}^{1}$ определяется из $(4.9), \varphi_{c}^{1}-$ из (4.10) и

$$
\begin{gathered}
\xi_{c}^{1} \equiv \xi_{n m}^{ \pm}\left(s_{c}^{1}, \vec{r}_{0}\right)=\varepsilon_{ \pm} l\left(s_{c}^{1}\right)^{-1} 2 L m-2 n\left\langle\vec{r}\left(s_{c}^{1}\right), \vec{l}\left(s_{c}^{1}\right)\right\rangle l\left(s_{c}^{1}\right)^{-2}, \\
\xi_{c}^{1}+2 n=\varepsilon_{ \pm} l\left(s_{c}^{1}\right)^{-1} 2 L m-2 n\left\langle\vec{r}_{0}, \vec{l}\left(s_{c}^{1}\right)\right\rangle l\left(s_{c}^{1}\right)^{-2} .
\end{gathered}
$$

Точка $z_{c}^{1}$ задается выбором знака $\varepsilon_{ \pm}$(или $\left.\varepsilon_{2}^{c}\right)$ и точки касания $\vec{r}\left(s_{c}^{1}\right)$. Напомним, что через $T(\partial \mathscr{D})$ обозначается объединение касательных прямых к контуру $\partial \mathscr{D}$ и $\vec{r}_{0} \in T(\partial \mathscr{D})$ - необходимое и достаточное условие существования критических точек первого типа. Пусть $s_{i}^{0}-$ точка уплощения, $p_{i}-$ ее порядок $(2.8)$ и $T_{0}=\bigcup T\left(s_{i}^{0}\right)-$ обгединение касательных $T\left(s_{i}^{0}\right)$, проведенных через точки уплощения $s_{i}^{0}$. Граница $\partial T$ множества $T(\partial \mathscr{D})=\bigcup T(s)$ состоит из дуг, высекаемых из $\partial \mathscr{D}$ некоторыми прямыми $T\left(s_{i}^{0}\right)$, и отрезков этих прямых. На рис. 3 заштрихованы области, не принадлежашие $T(\partial \mathscr{D})$, и указаны критические точки $s_{c}^{1} \equiv s_{c}^{1}\left(\vec{r}_{0}\right)$. Обратим внимание на очевидную аналогию рис. 3 и рис. 2 .

Основные факты о критических точках $s_{c}$ фазы $S$ следуют из явного вида производных

$$
\begin{gathered}
S^{(p)}(s)=F^{(1)} \varphi^{(1)}+\sum_{(\alpha)} F^{\left(\alpha_{1}+\cdots+\alpha_{p-1}\right)}\left(\varphi^{(1)}\right)^{\alpha_{1}} \cdots\left(\varphi^{(p-1)}\right)^{\alpha_{p-1}}, \\
F(\varphi)=-2 L m \varphi+2 n\left\langle\vec{r}_{0}, \vec{\eta}\right\rangle \quad\left(F^{(k)}=\left(\frac{d}{d \varphi}\right)^{k} F, \quad \varphi^{(k)}=\left(\frac{d}{d s}\right)^{k} \varphi\right), \\
(\alpha)=\left(\alpha_{1}, \ldots, \alpha_{p-1}\right), \quad \alpha_{1}+2 \alpha_{2}+\cdots+(p-1) \alpha_{p-1}=p,
\end{gathered}
$$




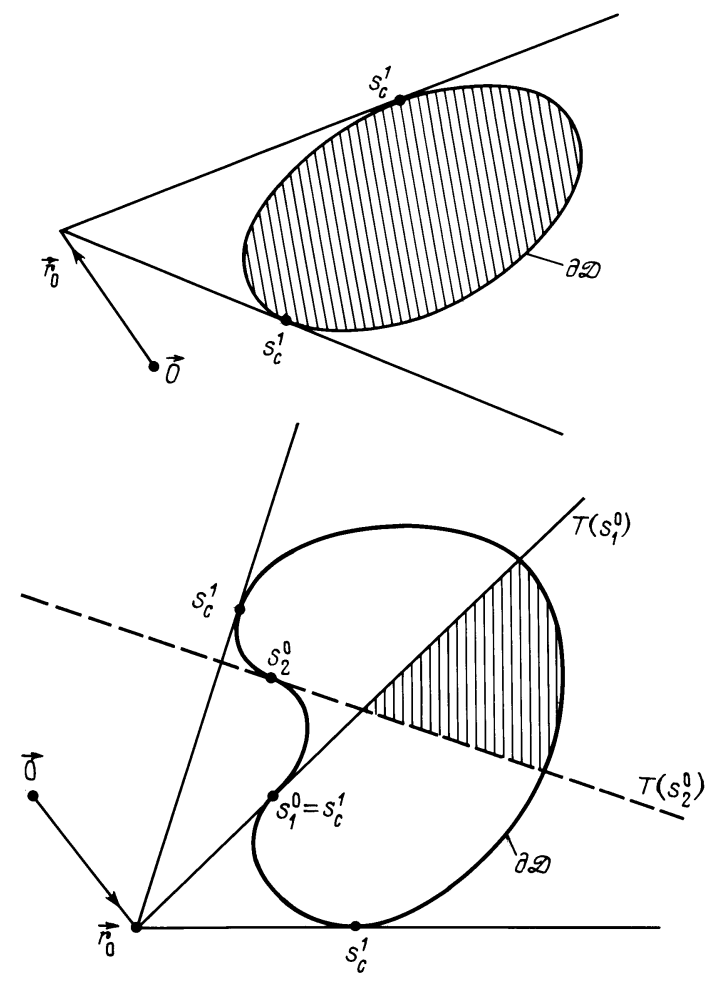

Рис. 3

с учетом того, что

$$
\varphi^{(m)}(s)=\frac{k^{(m-2)}(s)[\vec{l}, \vec{\nu}]}{l^{2}}+k^{(m-3)}(s) B_{m-3}^{m}+\cdots+k(s) B_{0}^{m}+[\vec{l}, \vec{\tau}] A_{m},
$$

где коэффициенты $B_{j}^{m}$ зависят от производных $k^{(p)}$ при $p \leqslant m-j$ и величины $A_{m}$ не зависят от $k$.

В частности, из этих формул следует, что при $\vec{r}_{0} \in T(\partial \mathscr{D}), \vec{r}_{0} \notin T_{0}$ все критические точки $s_{c}^{1}$ первого типа невырождены и

$$
S^{(2)}\left(s_{c}^{1}\right)=-\varepsilon_{ \pm} k\left(s_{c}^{1}\right)\left(\xi_{c}^{1}+2 n\right) .
$$

Если $\vec{r}_{0} \in T\left(s_{i}^{0}\right)$, то кроме невырожденных критических точек $s_{c}^{1} \neq s_{i}^{0}$ имеется вырожденная точка $s_{c}^{1}=s_{i}^{0}$, в которой

$$
\begin{aligned}
& S^{(m)}\left(s_{c}^{1}\right)=0 \quad\left(m \leqslant p_{i}+1, s_{c}^{1}=s_{i}^{0}\right), \\
& S^{\left(p_{i}+2\right)}\left(s_{c}^{1}\right)=-\varepsilon_{ \pm} k^{\left(p_{i}\right)}\left(s_{i}^{0}\right)\left(\xi_{c}^{1}+2 n\right) .
\end{aligned}
$$

Рассмотрим критические точки $s_{c}^{2}$ второго типа. Они определяются из условий

$$
\left\langle\vec{r}_{0}, \vec{\eta}^{*}\right\rangle=\gamma, \quad \vec{l}_{1}\left(s_{c}^{2}\right)=-\varepsilon_{ \pm} \vec{\eta}^{*}\left(s_{c}^{2}\right) \quad\left(\gamma \equiv \frac{L m}{n}\right) .
$$


Так как $\left|\vec{r}_{0}\right|<0.5$, то таких точек нет, если $|\gamma|>0.5$. В носителе амплитуды $u_{n}(1.35)$ их нет и при $n \geqslant 0$, так как $\xi \geqslant 0$ при $z \in \operatorname{supp} u_{n}(2.19)$. Определим угол $\alpha$ из условия

$$
\cos \alpha=\frac{|\gamma|}{\left|\vec{r}_{0}\right|} \quad\left(0 \leqslant \alpha \leqslant \frac{\pi}{2}\right) .
$$

Точки $s_{c}^{2}$ находятся следуюшим образом. Проведем, как показано на рис. 4 , через точку $\vec{r}_{0}$ две прямые под углами $\pm \alpha$ к прямой, соединяюшей начало координат $\vec{O}$ и точку $\vec{r}_{0}$, и припишем им значения $\varepsilon_{ \pm} \operatorname{sgn} \gamma, \varepsilon^{\prime}$. Четыре луча, выходящие из точки $\vec{r}_{0}$, нумеруются значениями $\varepsilon_{ \pm} \operatorname{sgn} \gamma, \varepsilon^{\prime}\left(\varepsilon^{\prime}= \pm 1\right)$. Точка $s_{c}^{2}$, отвечаюшая заданным значениям $\varepsilon_{ \pm} \operatorname{sgn} \gamma, \varepsilon^{\prime}$, сушествует, если соответствуюший луч пересекает $\partial \mathscr{D}$ и $\vec{r}\left(s_{c}^{2}\right) \in \partial \mathscr{D}-$ точка пересечения.

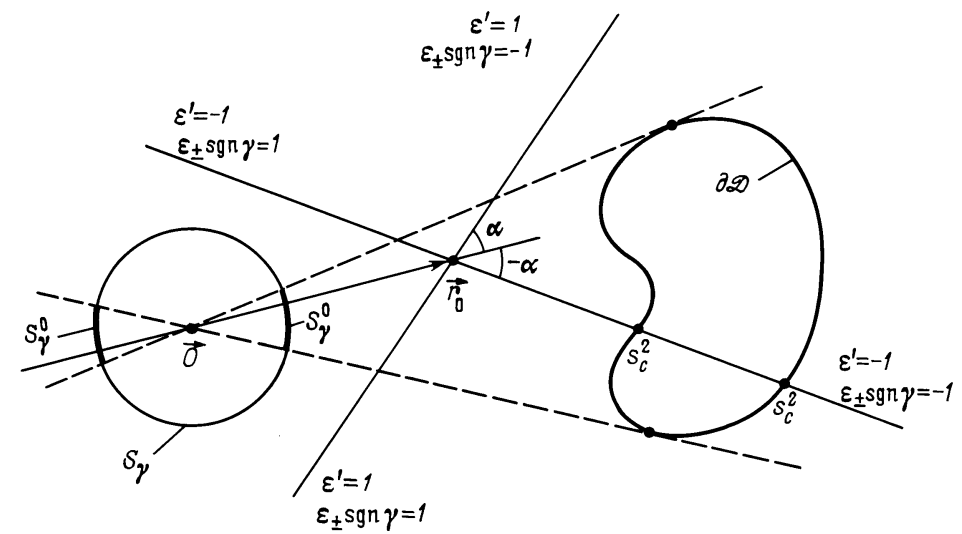

Рис. 4

Если при $\varepsilon_{ \pm}= \pm 1$ существуют (для заданного $\left.\gamma\right)$ критические точки $s_{c}^{2}$ второго типа, то мы говорим, что $\vec{r}_{0} \in \mathscr{P}_{\gamma}(\partial \mathscr{D})$. Необходимым условием сушествования точек $s_{c}^{2}$ является

$$
\left|\vec{r}_{0}\right| \geqslant|\gamma|, \quad|\gamma|<0.5
$$

Геометрия области $\mathscr{P}_{\gamma}(\partial \mathscr{D})$ будет рассмотрена при анализе точек $s_{c}^{3}$ третьего типа, а сейчас рассмотрим условия вырождения точек $s_{c}^{3}$. Через $S_{\gamma}$ обозначим окружность $\left|\vec{r}_{0}\right|=|\gamma|$ с иентром в начале координат $\vec{O}$ (см. рис. 4$)$. Пусть $\vec{r}_{0} \in S_{\gamma}$; будем говорить, что $\vec{r}_{0} \in S_{\gamma}^{0} \subset S_{\gamma}$, если прямая, соединяющая точки $\vec{r}_{0}$ и $\vec{O}$, пересекает $\partial \mathscr{D}$. Если $\vec{r}_{0} \in \mathscr{D}$, то $S_{\gamma}^{0}=S_{\gamma}$, а если $\vec{r}_{0} \notin \mathscr{D}$, то $S_{\gamma}^{0}$ есть объединение двух дуг, высекаемых из $S_{\gamma}$ внешними касательными к $\partial \mathscr{D}$, проведенными из начала координат. Если $\vec{r}_{0} \notin S_{\gamma}^{0}$, то $\left\langle\vec{r}_{0}, \vec{\eta}\left(s_{c}^{2}\right)\right\rangle \neq 0(\alpha \neq 0)$ и критические точки $s_{c}^{2}$ невырождены. В этом случае

$$
S^{(2)}\left(s_{c}^{2}\right)=2 n l^{-4}[\vec{l}, \vec{\tau}]^{2}\left[\vec{r}_{0}, \vec{\eta}\right]\left(s_{c}^{2}\right) .
$$

Если $\vec{r}_{0} \in S_{\gamma}^{0}$, то $\left\langle\vec{r}_{0}, \vec{\eta}\left(s_{c}^{2}\right)\right\rangle=0(\alpha=0)$ и критические точки $s_{c}^{2}$ вырождены $\left(S^{(2)}\left(s_{c}^{2}\right)=0\right)$, но $S^{(3)}\left(s_{c}^{2}\right) \neq 0$, точнее,

$$
S^{(3)}\left(s_{c}^{2}\right)=-2 n l^{-6}[\vec{l}, \vec{\tau}]^{3} \varepsilon_{3}^{c}\left|\vec{r}_{0}\right| \quad\left(\varepsilon_{3}^{c}= \pm 1,\left\langle\vec{r}_{0}, \vec{\eta}^{*}\left(s_{c}^{2}\right)\right\rangle=\varepsilon_{3}^{c}\left|\vec{r}_{0}\right|\right) .
$$


Напомним, что в силу предположения (4.6) при $\vec{r}_{0}=0$ точек второго типа нет, так как при этом $m \neq 0$.

Остается рассмотреть критические точки $s_{c}^{3}$ третьего типа. Так как это одновременно точки первого и второго типов, то при $s=s_{c}^{3}$ должны одновременно вьполняться условия (4.10) и $(4.16)$, т.е.

$$
\left\langle\vec{r}_{0}, \vec{\tau}\right\rangle=\gamma \varepsilon_{2}, \quad[\vec{l}, \vec{\tau}]=0
$$

Это возможно, только если точка $\vec{r}_{0}$ лежит на кривой $\Gamma_{\gamma}$, определяемой уравнением

$$
\vec{r}_{\Gamma}(s)=\vec{r}(s)-\vec{\tau}\langle\vec{r}, \vec{\tau}\rangle+\varepsilon_{2} \gamma \vec{\tau} \quad\left(\vec{r}_{\Gamma}(s) \in \Gamma_{\gamma}\right)
$$

При $\gamma=0$ это уравнение педальной кривой [24], для которой вектор $\vec{r}_{\Gamma}(s)-$ это нормаль из начала координат на касательную прямую $T(s)$ в точке $\vec{r}(s) \in \partial \mathscr{D}$. Кривая $\Gamma_{\gamma}=\Gamma_{\gamma}(\partial \mathscr{D}, \vec{O})$ зависит от взаимного расположения контура $\partial \mathscr{D}$ и начала координат. Способ ее построения ясен из определения вектора $\vec{r}_{\Gamma}(s) \in \Gamma_{\gamma}(4.20)$.

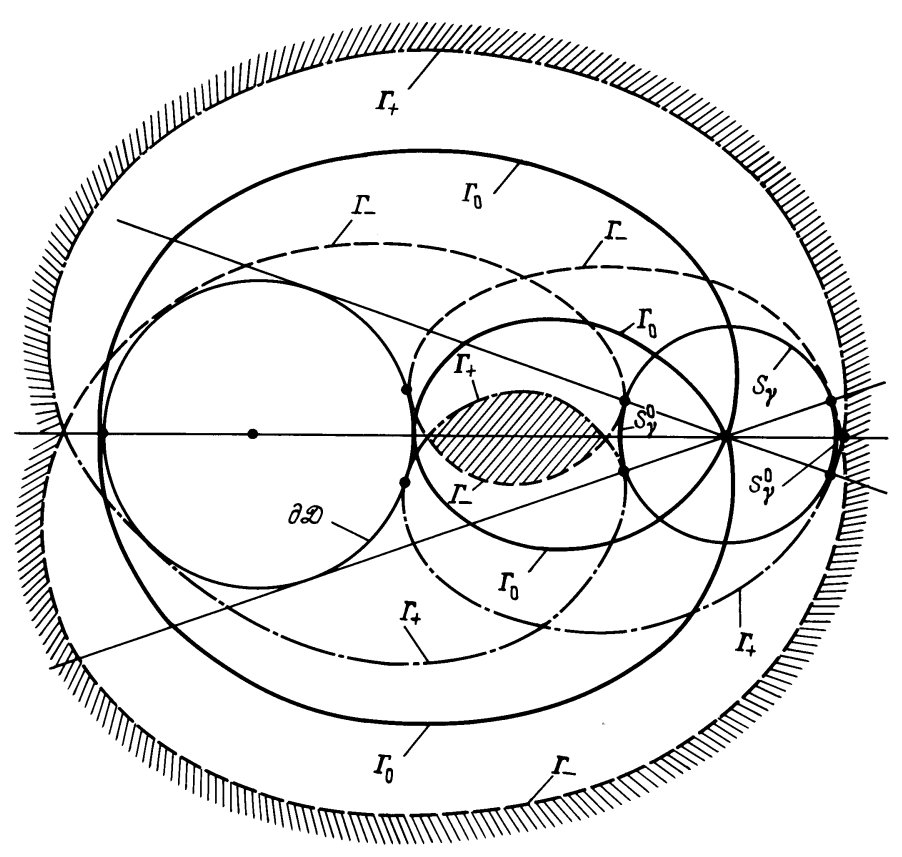

Рис. 5

На рис. 5 представлены кривые $\Gamma_{0}, \Gamma_{ \pm}$в случае, когда $\mathscr{D}-$ круг и $\vec{r}_{0} \notin \mathscr{D}$. Кривая $\Gamma_{0}$ отвечает $\gamma=0$, а кривые $\Gamma_{ \pm}-\varepsilon_{2} \gamma= \pm|\gamma|$. Эти кривые составляют границу $\partial \mathscr{P}_{\gamma}$ области $\mathscr{P}_{\gamma}(\partial \mathscr{D})$, где есть точки второго типа. На рис. 5 заштрихована область, где таких точек нет. Отметим, что кривые $\Gamma_{\gamma}$ и $S_{\gamma}$ не пересекаются, но могут касаться друг друга, например, в крайних точках дуг $S_{\gamma}^{0}$. Обозначим через $T(\vec{O})$ облединение касательных к $\partial \mathscr{D}$, проведенных из начала координат $\vec{O}$. Тогда $T(\vec{O}) \cap S_{\gamma} \subset S_{\gamma}^{0}$ 
и $\Gamma_{\gamma}$ касается $S_{\gamma}$ в точках $T(\vec{O}) \cap S_{\gamma}$. Если на контуре $\partial \mathscr{D}$ имеется точка уплощения $s_{i}^{0}$, то $\vec{r}_{\Gamma}\left(s_{i}^{0}\right)$ - точка возврата кривой $\Gamma_{\gamma}$, лежащая на прямой $T\left(s_{i}^{0}\right)$. На двойной касательной к $\partial \mathscr{D}$ лежит точка самопересечения кривой $\Gamma_{\gamma}$. Таким образом, при прохождении дуги $\left(s_{1}, s_{2}\right)$ между точками с обшей касательной $\left(T\left(s_{1}\right)=T\left(s_{2}\right)\right)$ в $\Gamma_{\gamma}$ вклеивается граница луночки, как показано на рис. 6.

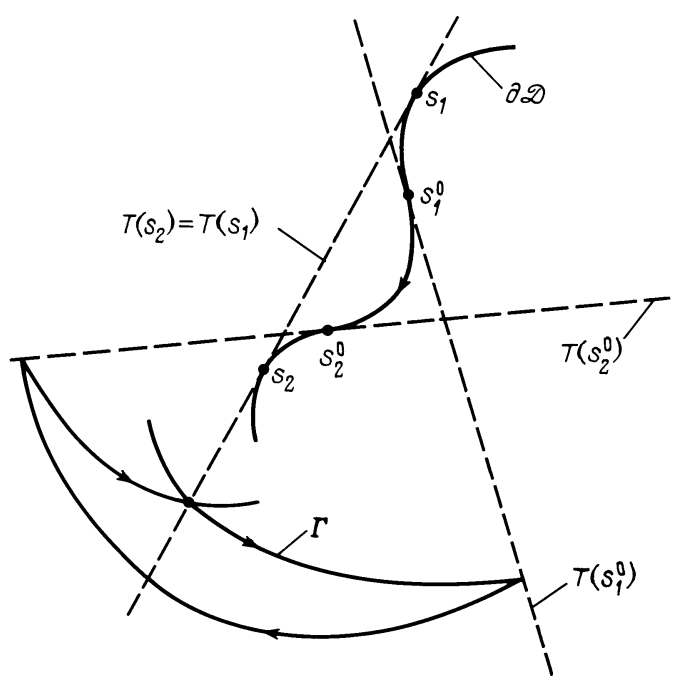

Рис. 6

Заметим, что если $\vec{r}_{\Gamma}(s) \notin \partial \mathscr{D}$, то касательная к $\Gamma_{\gamma}$ в точке $\vec{r}_{\Gamma}(s)$ не совпадает с $\vec{\tau}(s)$, т.е. $T(s)$ трансверсально пересекает $\Gamma_{\gamma}$ в точке $\vec{r}_{\Gamma}(s)$.

Пусть $\vec{r}_{0} \in \Gamma_{\gamma}$ и, следовательно, имеются критические точки третьего типа. Они всегда вырождены, и каждая из них получается слиянием по крайней мере двух точек второго типа. Для заданного $\vec{r}_{0} \in \Gamma_{\gamma}$ могут существовать несколько точек третьего типа различной кратности вырождения.

Прямое вычисление с помошью формул (4.22), (4.13) показьвает, что все варианты исчерпываются следующими случаями.

1. Пусть $\vec{r}_{0} \in \Gamma_{\gamma}, \vec{r}_{0} \notin T_{0}, \vec{r}_{0} \notin T(\vec{O}) \cap S_{\gamma}$. Тогда для всех точек $s_{c}^{3}$

$$
S^{(m)}\left(s_{c}^{3}\right)=0 \quad(m \leqslant 3), \quad S^{(4)}\left(s_{c}^{3}\right)=-\frac{3 k^{2}}{l^{2}} 2 n \varepsilon_{2}\left\langle\vec{r}_{0}, \vec{\nu}\right\rangle\left(s_{c}^{3}\right) .
$$

2. Пусть $\vec{r}_{0} \in \Gamma_{\gamma}, \vec{r}_{0} \notin T_{0}, \vec{r}_{0} \in T(\vec{O}) \cap S_{\gamma}$. Тогда для всех точек $s_{c}^{3}$

$$
S^{(m)}\left(s_{c}^{3}\right)=0 \quad(m \leqslant 5), \quad S^{(6)}\left(s_{c}^{3}\right)=15 k^{3}\left(s_{c}^{3}\right) 2 n \varepsilon_{3}\left|\vec{r}_{0}\right| .
$$

3. Пусть $\vec{r}_{0} \in \Gamma_{\gamma} \cap T\left(s_{i}^{0}\right), \vec{r}_{0} \notin T(\vec{O}) \cap S_{\gamma}$. Тогда сушествует точка $s_{c}^{3}=s_{i}^{0}$ такая, чTо

$$
\begin{aligned}
S^{(m)}\left(s_{i}^{0}\right) & =0 \quad\left(m \leqslant 2 p_{i}+3\right) \\
S^{\left(2 p_{i}+4\right)}\left(s_{i}^{0}\right) & =-2 n \varepsilon_{2}\left(\frac{\left\langle\vec{r}_{0}, \vec{\nu}\right\rangle k^{\left(p_{i}\right)}}{l^{2}}\right)\left(s_{i}^{0}\right) \frac{\left(2 p_{i}+1\right) !}{2\left(\left(p_{i}+2\right) !\right)^{2}} .
\end{aligned}
$$


Кроме того, возможно, существуют точки $s_{c}^{3}$, описанные в п. 1.

4. Пусть $\vec{r}_{0} \in \Gamma_{\gamma} \cap T\left(s_{i}^{0}\right), \vec{r}_{0} \in T(\vec{O}) \cap S_{\gamma}$. Тогда сушествует точка $s_{c}^{3}=s_{i}^{0}$ такая, что

$$
\begin{aligned}
S^{(m)}\left(s_{i}^{0}\right) & =0 \quad\left(m \leqslant 3 p_{i}+5\right), \\
S^{\left(3 p_{i}+6\right)}\left(s_{i}^{0}\right) & =\varepsilon_{2} \varepsilon_{ \pm} \varepsilon_{3} \frac{\left(2 p_{i}+6\right) ! 2 n}{6\left(\left(2 p_{i}+2\right) !\right)^{2}} \frac{\left(k^{\left(p_{i}\right)}\left(s_{i}^{0}\right)\right)^{3}}{l\left(s_{i}^{0}\right)^{3}},
\end{aligned}
$$

и, возможно, существуют точки $s_{c}^{3}$, описанные в п. 2 .

Случай 4 реализуется, только если касательные к $\partial \mathscr{D}$, проведенные из начала координат, проходят через точки уплощения, т.е. $T_{0} \cap T(\vec{O}) \neq \varnothing$.

Сформулируем полученные результаты в виде леммы.

ЛЕмма 4.1. Пусть $d\left(\vec{r}_{0}, \partial \mathscr{D}\right)>0,(m, n) \neq(0,0), \partial \mathscr{D} \in\left\{\partial^{\omega}\right\}, \vec{r}_{0} \neq 0$ илu $m \neq 0$. Тогда

1) все критические точки $z_{c}$ фазы $\Phi(4.1)$ имеют corank $\leqslant 1$, и все особенности фазы $\Phi$ принадлежат к классам $A_{k}$;

2) критические точки могут быть вырождены тогда и только тогда, когда

$$
\vec{r}_{0} \in T_{0} \cup \Gamma_{\gamma} \cup S_{\gamma}^{0} ;
$$

3) порядок особенности $A_{k}$ определяется кратностью вырождения критических точек функиии $S$, задаваемой соотношениями (4.5), и соответствующие $k$ и $S^{(k+1)}\left(s_{c}\right)$ указаны выше и задаются соотношениями (4.14), (4.15), (4.17), (4.18), (4.21)-(4.24).

\section{§. Оценка ошибки дискретизации}

5.1. Предварительные замечания. Исходньм пунктом доказательства теоремы 2.1 служит соотношение (3.26), в котором величину $\Delta \mu_{T F}^{A}\left(\vec{r}_{0}\right)(3.25)$ можно представить в виде

$$
\begin{gathered}
\Delta \mu_{T F}^{A}\left(\vec{r}_{0}\right)=\frac{i \Omega}{2 \pi} \sum_{n, m}^{F} J_{n m}^{B} e^{i 2 n \Omega \delta}, \\
J_{n m}^{B} \equiv J_{m}^{B}\left[u_{n}\right]=\int_{0}^{B} d \xi \int_{0}^{2 \pi} d \varphi \int_{\partial \mathscr{D}} d s e^{i \Omega \Phi_{n m}}=u_{n} .
\end{gathered}
$$

Отсюда следует, что

$$
\left|\Delta \mu_{T F}^{A}\left(\vec{r}_{0}\right)\right| \leqslant \frac{\Omega}{2 \pi} \sum_{n, m}^{F}\left|J_{n m}^{B}\left[u_{n}\right]\right|,
$$

и вопрос сводится к оценке ОИ $J_{n m}^{B}\left[u_{n}\right]$. Чтобы преодолеть технические трудности, связанные с негладкостью амплитуды $u_{n}(1.35)$, и рассматривать вклады точек нарушения гладкости по отдельности, введем разбиение единицы

$$
1=\widetilde{\rho}(\xi)+\rho_{0}(\xi)+\sum_{\xi_{i}^{n} \neq(0, B)} \rho_{i}(\xi)+\rho_{B}(\xi), \quad \rho_{0}, \rho_{i}, \rho_{B} \in C_{0}^{\infty},
$$


с центрами в точках нарушения гладкости $0, \xi_{i}^{n}, B$ и масштабами $a_{0}, a_{i}, a_{B}$,

$$
\rho_{t}(\xi)=\left\{\begin{array}{ll}
1 & \left(\left|\xi-\xi_{t}\right| \leqslant a_{t}\right), \\
0 & \left(\left|\xi-\xi_{t}\right| \geqslant 2 a_{t}\right),
\end{array} \quad\left|\rho_{t}^{(k)}(\xi)\right| \leqslant C_{k} a_{t}^{-k} \quad(t=0, i, B) .\right.
$$

Предполагается, что $\rho_{t} \in C_{0}^{\infty}$ и (см. (2.4))

$$
\min \left\{a_{0}, a_{i}, a_{B}\right\} \geqslant a>0, \quad 4 a_{0}<\Delta \xi, \quad 4 a_{i}<\Delta \xi, \quad 4 a_{B}<\Delta \xi .
$$

В этом случае носители функций $\rho_{0}, \rho_{i}, \rho_{B}$ не пересекаются, и равенство (5.3) служит определением функции $\widetilde{\rho}$. Запишем интеграл $J_{n m}^{B}\left[u_{n}\right]$ в виде

$$
J_{n m}^{B}\left[u_{n}\right]=J_{n m}\left[\widetilde{\rho} u_{n}\right]+J_{n m}\left[\rho_{0} u_{n}\right]+\sum_{\xi_{i}^{n} \neq(0, B)} J_{n m}\left[\rho_{i} u_{n}\right]+J_{n m}\left[\rho_{B} u_{n}\right] .
$$

Здесь и ниже верхний индекс $B$ у интегралов $J_{n m}^{B}\left[\widetilde{\rho} u_{n}\right], J_{n m}^{B}\left[\rho_{B} u_{n}\right]$ опускается. Интегралы в правой части (5.6) оцениваются по отдельности, и метод их оценки зависит от того, какая из двух областей $-D_{\beta_{1} \beta_{2}}^{1} \cup D_{\beta_{1} \beta_{2}}^{2}(2.1)$ или $D_{\beta_{1} \beta_{2}}^{3}$ - рассматривается. Основное утверждение состоит в том, что результатов, приведенных в приложсении, достаточно для доказательства теоремы 2.1. Использование этих результатов требует рутинных, но достаточно трудоемких вычислений, которые мы не приводим, хотя все необходимые оценки и условия их применимости будут выписаны. При этом наша цель состоит не в получении наилучших оценок, а в демонстрации возможности явно провести все эти оценки и вьписать условия их применимости, что и позволяет убедиться в непротиворечивости этих условий.

При применении результатов из приложения мы без дополнительных оговорок будем использовать введенные там обозначения. Ссылки на утверждения и формуль из приложения снабжень буквой $\Pi^{1}$.

Введем некоторые определения, которые будут использоваться ниже. Предположим, что удалось получить представление вида

$$
\Delta \mu_{T}^{A}\left(\vec{r}_{0}\right)=\mu_{0}\left(\vec{r}_{0}\right)+\mu_{1}\left(\vec{r}_{0}\right) \text { и }\left|\mu_{1}\left(\vec{r}_{0}\right)\right| \leqslant \begin{cases}R & \left(\vec{r}_{0} \in D_{\beta_{1} \beta_{2}}^{1} \cup D_{\beta_{1} \beta_{2}}^{2}\right), \\ R_{1} & \left(\vec{r}_{0} \in D_{\beta_{1} \beta_{2}}^{3}\right),\end{cases}
$$

в котором величины $R, R_{1}$ удовлетворяют оценкам (2.28), (2.31). В әтом случае будем говорить, что величина $\mu_{1}$ допускает удовлетворительную оценку или что $\mu_{1}$ не вносит основного вклада в оценку $\Delta \mu_{T}^{A}\left(\vec{r}_{0}\right)$. Этот факт будет записьваться в виде $\mu \in\{O\}$. Аналогично, если $J_{n m}=J_{n m}^{0}+J_{n m}^{1}$ и при этом

$$
\left|J_{n m}^{1}\right| \leqslant \bar{J}_{n m}^{1} \quad u \quad \Omega \sum_{n, m}^{F} \bar{J}_{n m}^{1} \leqslant \begin{cases}C \gamma(\ln \Omega) \Omega^{-0.5-\varepsilon} & \left(\vec{r}_{0} \in D_{\beta_{1} \beta_{2}}^{1} \cup D_{\beta_{1} \beta_{2}}^{2}\right), \\ C \gamma \Omega^{-2} & \left(\vec{r}_{0} \in D_{\beta_{1} \beta_{2}}^{3}\right)\end{cases}
$$

то мы говорим, что оченка $\left|J_{n m}^{1}\right| \leqslant \bar{J}_{n m}^{1}$ удовлетворительна или что $J_{n m}^{1} \in\{O\}$ и величина $J_{n m}^{1}$ не вносит основного вклада в оценку $\Delta \mu_{T}^{A}\left(\vec{r}_{0}\right)$. Пусть для величины $J_{n m}^{1}$ получена оценка вида

$$
\left|J_{n m}^{1}\right| \leqslant \bar{J}_{n m}^{1}=C\left(\varkappa, j_{0}, j_{1}\right)|n|^{k} A(n) F,
$$

\footnotetext{
${ }^{1}$ Список обозначений приведен в конце приложения.
} 
где значение $A(n)$ определено в $(3.4)$ и $\varkappa>k+1$. Тогда

$$
\Omega \sum_{n, m}^{F} \bar{J}_{n m}^{1} \leqslant C\left(\varkappa, j_{0}, j_{1}\right) A_{0} \frac{\gamma N_{1}}{L} F
$$

и оценка (5.8) удовлетворительна $\left(J_{n m}^{1} \in\{O\}\right)$, если

$$
\Omega \frac{A_{0} N_{1}}{L} F \leqslant \begin{cases}(\ln \Omega) \Omega^{-0.5-\varepsilon} & \left(\vec{r}_{0} \in D_{\beta_{1} \beta_{2}}^{1} \cup D_{\beta_{1} \beta_{2}}^{2}\right), \\ \Omega^{-2} & \left(\vec{r}_{0} \in D_{\beta_{1} \beta_{2}}^{3}\right) .\end{cases}
$$

Ниже используется именно этот грубый критерий удовлетворительности оценок.

В $\S 2$ были определены величины $\beta_{i}>0(i=1,2,3)$. Введем $\beta_{4}, \beta_{5}, \beta_{6}\left(\beta_{i}>0\right)$ и положим $d \equiv d\left(r_{0}, \partial \mathscr{D}\right) \geqslant d_{0}$ и

$$
\begin{aligned}
d_{0} & =C \Omega^{-\beta_{1}}, \quad D_{0}=C \Omega^{-\beta_{2}}, \quad D^{\prime}=C \Omega^{-\beta_{3}}, \quad N_{1}=C \Omega^{\beta_{4}}, \\
a & =C \Omega^{-\beta_{5}}, \quad a_{0}=C \Omega^{-\beta_{6}}, \quad \beta_{i}>0, \quad \beta_{2}>\beta_{3}, \quad \beta_{5} \geqslant \beta_{6} .
\end{aligned}
$$

Величины $B, M$ в соответствии с (3.27) выбираются из условий

$$
B=N_{1}, \quad 0.9 M=\gamma N_{1} L^{-1} \quad(\gamma \geqslant 1) .
$$

В этом случае из (3.28) следует (например, при $\left.\varkappa_{1} \geqslant 1.5, \varkappa_{2} \geqslant 0.5\right)$, что

$$
\left|\Delta_{F} \mu^{A}\left(\vec{r}_{0}\right)\right| \leqslant C \mathscr{L} A_{0}\left[N_{1}^{-(\varkappa-1)}+e^{-\gamma N_{1} \Omega}\right],
$$

и эта оценка удовлетворительна $\left(\Delta_{F} \mu^{A}\left(r_{0}\right) \in\{O\}\right)$, если (см. (5.9))

$$
(\varkappa-1) \beta_{4} \geqslant \begin{cases}C \alpha+1.5+\varepsilon & \left(\vec{r}_{0} \in D_{\beta_{1} \beta_{2}}^{1} \cup D_{\beta_{1} \beta_{2}}^{2}\right), \\ C \alpha+3 & \left(\vec{r}_{0} \in D_{\beta_{1} \beta_{2}}^{3}\right) .\end{cases}
$$

Непосредственно из определения интеграла $J_{n m}\left[\rho_{B} u_{n}\right]$ следует, что при условиях

$$
4 a_{B} \leqslant B, \quad e^{2 \tau_{1} a_{B}} \leqslant C
$$

которые ниже предполагаются выполненными, имеет место оценка

$$
\left|J_{n m}\left[\rho_{B} u_{n}\right]\right| \leqslant C \mathscr{L} A(n) e^{-\tau_{1} N_{1}} N_{1}^{-\varkappa 1},
$$

которая удовлетворительна при тех же условиях (5.13).

Таким образом, остается оценить интегралы $J_{n m}\left[\rho_{t} u_{n}\right] \quad\left(\rho_{t}=\widetilde{\rho}, \rho_{0}, \rho_{i}\right)$ и показать, что все условия справедливости полученных оценок совместны при $\beta_{i}>0$ $(i=1, \ldots, 6), \alpha>0, \varepsilon>0$. Заметим, что требование $\beta_{5} \geqslant \beta_{6}>0$ позволяет сушественно упростить оценки вклада точек нарушения гладкости. Пункты 1,2 из теоремы 2.1 являются прямьм следствием более точных оценок, приведенных соответственно в пунктах 1,2 следующей леммы. 
Лемма 5.1. Пусть $\mu=\chi_{\mathscr{D}} \in\left\{\mu^{\omega}, I\right\}, A \in\{A\}$. Тогда можно указать числа $\alpha>0, \varepsilon_{0}>0, \beta_{i}>0 \quad(i=1, \ldots, 6), \varkappa_{0}, j_{0}, j_{1}$ такие, что при выполнении условий (2.26) для любого достаточно гладкого алгоритма А справедливь следующие утверждения.

1. При $\vec{r}_{0} \in D_{\beta_{1} \beta_{2}}^{1}$ оченка величины $\Delta \mu_{T}^{A}\left(\vec{r}_{0}\right)$ определяется вкладом критических точек $s_{c}^{1}\left(\vec{r}_{0}\right)$ первого типа, т.е.

$$
\left|\Delta \mu_{T}^{A}\left(\vec{r}_{0}\right)\right| \leqslant \sum_{\substack{|n|=0 \\(n, m) \neq(0,0)}}^{N_{1}} \sum_{\substack{m \mid=0 \\\left(n, s_{0}^{1}\left(\vec{r}_{0}\right)\right.}}^{M-1} \sum_{ \pm} \frac{C\left|w_{n m}^{ \pm}\left(s_{c}^{1}, \vec{r}_{0}\right)\right|}{\left|\Omega k\left(s_{c}^{1}\right)\right|^{1 / 2}\left|\xi_{n m}^{ \pm}\left(s_{c}^{1}\right)+2 n\right|}+R
$$

и для $R$ выполняется оченка (2.28). Суммирование в этой формуле ведется по всем $s_{c}^{1}\left(\vec{r}_{0}\right), \pm, n, m$ таким, что

$$
\left|\xi_{n m}^{ \pm}\left(s_{c}^{1}\right)+2 n\right| \geqslant a, \quad \xi_{n m}^{ \pm}\left(s_{c}^{1}\right) \geqslant 0,
$$

а функиии $w_{n m}^{ \pm}$задаются равенствами

$$
w_{n m}^{ \pm}\left(s, \vec{r}_{0}\right)= \pm\langle\vec{\tau}(s), \vec{l}(s)\rangle l(s)^{-2} v_{n}\left(\xi_{n m}^{ \pm}(s)\right) .
$$

Величиньи $\xi_{n m}^{ \pm}\left(s_{c}^{1}\right) \equiv \xi_{n m}^{ \pm}\left(s_{c}^{1}\left(\vec{r}_{0}\right), \vec{r}_{0}\right)$ определеньи в $(4.11)$.

2. При $\vec{r}_{0} \in U\left(\beta_{2}, T\left(s_{j}^{0}\right)\right),\left|\vec{r}_{0}-\vec{r}_{m}^{0}\right| \geqslant D^{\prime}$ оченка определяется вкладом критических точек $s_{c}^{1}\left(\vec{r}_{0 j}\right) \neq s_{j}^{0}$ и точки уплощения $s_{j}^{0}\left(\vec{r}_{m}^{0}-\right.$ точки пересечения прямых $\left.T\left(s_{i}^{0}\right)(2.30)\right)$ :

$$
\begin{aligned}
\left|\Delta \mu_{T}^{A}\left(\vec{r}_{0}\right)\right| \leqslant & \sum_{|n|=0}^{N_{1}} \sum_{|m|=0}^{M-1} \sum_{ \pm}\left[\sum_{s_{c}^{1}\left(r_{0 j}\right)} \frac{C\left|w_{n m}^{ \pm}\left(s_{c}^{1}\left(r_{0 j}\right), \vec{r}_{0 j}\right)\right|}{\left|\Omega k\left(s_{c}^{1}\left(r_{0 j}\right)\right)\right|^{1 / 2}\left|\xi_{n m}^{ \pm}\left(s_{c}^{1}\left(r_{0 j}\right), r_{0 j}\right)+2 n\right|^{1 / 2}}\right. \\
& \left.+\frac{C B_{j}\left[w_{n m}^{ \pm}\right]}{\left(\Omega K_{j}\left|\xi_{n m}^{ \pm}\left(s_{j}^{0}, r_{0 j}\right)+2 n\right|\right)^{1 /\left(p_{j}+1\right)}}\right]+R .
\end{aligned}
$$

В этой формуле величина $B_{j}\left[w_{n m}^{ \pm}\right]$удовлетворяет оценке

$$
B_{j}\left[w_{n m}^{ \pm}\right] \leqslant\left|w_{n m}^{ \pm}\left(s_{j}^{0}, \vec{r}_{0 j}\right)\right|+C \overline{\partial_{t} w_{n m}^{ \pm}} D_{0}+C \overline{\left(w_{n m}^{ \pm}\right)^{(1)}} \delta_{j}+C d_{0}^{-1} A(n) a^{j_{0}},
$$

в которой $\delta_{j} \leqslant C \Omega^{-\eta_{j}}, \eta_{j}>0$ u $\vec{r}_{0 j}-$ проекция $\vec{r}_{0}$ на $T\left(s_{j}^{0}\right), \overline{\left(w_{n m}^{ \pm}\right)^{(1)}}-$ верхняя оченка производной $\left(w_{n m}^{ \pm}\right)^{(1)}$ nо $s, \partial_{t}$ - оператор дифференцирования по параметрам $t=\vec{r}_{0}=\left(x_{0}, y_{0}\right)$, а определено в (5.5), (5.10) и для $R$ верна оченка (2.28).

5.2. Доказательство леммы 5.1. На формальном уровне равенства

$$
\begin{aligned}
& J_{n m}\left[u_{n}\right]=\frac{2 \pi}{\Omega} \sum_{ \pm} I_{1}^{ \pm}\left[w_{n m}^{ \pm}\right]+\Delta J_{n m}, \\
& I_{1}^{ \pm}\left[w_{n m}^{ \pm}\right]=\int_{\partial \mathscr{D}} e^{i \Omega S_{ \pm}(s)} w_{n m}^{ \pm}(s) d s
\end{aligned}
$$

сразу получаются, если в интеграле $J_{n m}\left[u_{n}\right]$ проинтегрировать по $\varphi, \xi$ с помошњю метода стационарной фазы [31], (П.15) (критические точки $\varphi_{ \pm}, \xi_{ \pm}$в (П.33) - невырождены - (4.4)) и при этом ограничиться главным критическим вкладом (П.15). Пока будем понимать равенство (5.18) как определение величины $\Delta J_{n m}$, а ниже будет показано, что $\Delta J_{n m} \in\{O\}$. 
5.2.1. Одномерный интеграл $I_{1}[u]$. Рассмотрим одномерный интеграл $I_{1}^{ \pm}\left[w_{n m}^{ \pm}\right]$(5.19). Будем использовать обозначения $(5.10)$, и, таким образом, $D_{\beta_{1} \beta_{2}}^{1}=$ $\left\{\vec{r}_{0} \in D \cap T(\partial \mathscr{D}) \mid d\left(\vec{r}_{0}, \partial \mathscr{D}\right) \geqslant d_{0}, d\left(\vec{r}_{0}, T_{0}\right) \geqslant D_{0}\right\}, D_{\beta_{1} \beta_{2}}^{2}=\left\{\vec{r}_{0} \in D \mid d\left(\vec{r}_{0}, \partial \mathscr{D}\right) \geqslant d_{0}\right.$, $\left.d\left(r_{0}, T_{0}\right) \leqslant D_{0}\right\}$, а величины $D_{0}, d_{0}$ можно рассматривать как параметры. Условие достаточной гладкости позволяет упростить оценки и не рассматривать критические точки второго и третьего типов. Заметим, что вклад этих точек также может быть оценен на основе результатов, изложенных в приложении. Введем функции

$$
\chi_{n m}^{ \pm}(s)=0 \quad(n \geqslant 0), \quad \chi_{n m}^{ \pm}(s)= \begin{cases}1, & \left|\xi_{n m}^{ \pm}(s)+2 n\right| \leqslant a, \\ 0, & \left|\xi_{n m}^{ \pm}(s)+2 n\right|>a,\end{cases}
$$

и представим интеграл $I_{1}^{ \pm}\left[w_{n m}^{ \pm}\right]$в виде

$$
I_{1}^{ \pm}\left[w_{n m}^{ \pm}\right]=I_{1}^{ \pm}\left[\left(1-\chi_{n m}^{ \pm}\right) w_{n m}^{ \pm}\right]+I_{1}^{ \pm}\left[\chi_{n m}^{ \pm} w_{n m}^{ \pm}\right] .
$$

Второй интеграл в правой части оценим тривиально:

$$
\left|I_{1}^{ \pm}\left[\chi_{n m}^{ \pm} w_{n m}^{ \pm}\right]\right| \leqslant C A(n) \mathscr{L} d_{0}^{-1} a^{j_{0}} .
$$

Чтобы оценить интегралы $I_{1}^{ \pm}\left[\left(1-\chi_{n m}^{ \pm}\right) w_{n m}^{ \pm}\right]$, рассмотрим общий ОИ

$$
I_{1}(u)=\int_{\partial \mathscr{D}} u e^{i \Omega S} d s \quad\left(S=S_{ \pm} ; \text {см. }(4.5)\right) .
$$

Предполагая наличие множителя $\left(1-\chi_{n m}^{ \pm}\right)$в амплитуде, ограничимся его оценкой в специальном случае, когда (см. (4.7), (5.5))

$$
\left|S^{(1)}(s)\right| \geqslant|\Psi(s)|, \quad \Psi(s)=a[\vec{l}(s), \vec{\tau}(s)] .
$$

Критические точки первого типа совпадают с нулями функщии $\Psi$.

Для оценки ОИ $I_{1}[u]$ используем леммы П.1, П.2. Как и везде ниже, приведем только окончательные результаты. Методика их получения продемонстрирована на ряде примеров в работе [25].

С помошью формул Серре- $\Phi$ рене (2.10) и (2.7) получим, что

$$
\left|\varphi^{(n)}(s)\right| \leqslant C_{n} \frac{\bar{k} b^{n-2}}{d_{0}^{n}}, \quad\left|S^{(n)}(s)\right| \leqslant C_{n} \frac{N_{1} \bar{k} b^{n-2}}{d_{0}^{n}} .
$$

Для числа $\mathscr{N}_{1}$ критических точек $s_{c}^{1}$ справедлива оценка (см. (2.9)):

$$
\mathscr{N}_{1} \leqslant C \frac{\mathscr{L}}{\min \left\{\Delta_{i}\right\}} \leqslant C \mathscr{L} \bar{k} b^{p+1} K_{0}^{-1} .
$$

Нужные оценки снизу для величин $k\left(s_{c}^{1}\right)$ получаются с помощью леммы П.3. Рассмотрим трубчатые окрестности $T_{D_{i}}$ прямых $T\left(s_{i}^{0}\right) \quad\left(T_{D_{i}}=\left\{\vec{r}_{0} \mid d_{i} \leqslant D_{i}\right\}\right.$, $\left.d_{i}=d\left(\vec{r}_{0}, T\left(s_{i}^{0}\right)\right)\right)$ и будем предполагать, что $D_{i} \geqslant D_{0}$. Пусть выполнены условия

$$
\begin{gathered}
D_{i} \leqslant C_{i}\left(\frac{d_{0} K_{i}}{E_{i}}\right)^{p_{i}+2}, \quad\left(\frac{D_{i}}{d_{0} K_{i}}\right)^{\frac{1}{p_{i}+1}} \leqslant C_{i} \Delta_{i}, \\
K_{i}^{\frac{1}{p_{i}+1}} D_{i}^{\frac{p_{i}+2}{p_{i}+1}} \leqslant C_{i} \widetilde{k}, \quad E_{i} \equiv C_{i} \mathscr{L}^{p_{i}+3}(\bar{k})^{2} b^{2 p_{i}+3} \quad\left(C_{i} \equiv C\left(p_{i}\right)\right) .
\end{gathered}
$$


Тогда если $\vec{r}_{0} \in T_{D_{i}}(\forall i)$, то с помощью леммы П.3 получим

$$
\left|k\left(s_{c}^{1}\right)\right| \geqslant \min _{i}\left\{C_{i} K_{i}^{\frac{1}{p_{i}+1}} D_{i}^{\frac{p_{i}}{p_{i}+1}}\right\} \geqslant k_{D}, \quad k_{D}=C K_{0}^{1 / 2} D_{0} .
$$

Если $\vec{r}_{0} \in T_{D_{j}}$, то для “близких" в смысле леммы П.3 к $s_{j}^{0}$ точек $s_{c}^{1}$

$$
k\left(s_{c}^{1}\right) \asymp C_{j} K_{j}\left(\frac{d_{j}}{d\left(\vec{r}_{0}, \vec{r}\left(s_{j}^{0}\right)\right)}\right)^{\frac{p_{j}}{p_{j}+2}},
$$

а для всех остальных точек

$$
k\left(s_{c}^{1}\right) \geqslant k_{d} \equiv \min _{i}\left\{C K_{i}\left(\frac{d_{0} K_{i}}{E_{i}}\right)^{p_{i}}, \widetilde{k}\right\} .
$$

Рассмотрим область $\vec{r}_{0} \in D_{\beta_{1} \beta_{2}}^{1}$. Условия (5.24) при $D_{i}=D_{0}$ выполняются, если

$$
\beta_{2} \geqslant C_{1}(p) \alpha+C_{2}(p) \beta_{1} \text {. }
$$

Экстремальные точки $s_{\mathrm{ex}}$ функции $\Psi=[\vec{l}, \vec{r}]\left(\Psi^{(1)}\left(s_{\mathrm{ex}}\right)=0\right)$ легко находятся: $s_{\mathrm{ex}}=$ $\left(s_{i}^{0}, s_{\mathrm{ex}}^{1}\right) \quad\left([\vec{l}, \vec{\tau}]\left(s_{\mathrm{ex}}^{1}\right)=0\right)$. Отсюда следует, что в (П.23) можно взять $\Psi_{\Delta}=C a D_{0}$. Используя формулы (4.12), (4.13), удается показать, что в оценке (П.23) можно положить

$$
\Delta_{c}=C \frac{k_{D} d_{0}^{2}}{\bar{k} b}, \quad \delta_{c}=\delta_{0}=C \frac{k_{D} d_{0}^{4} a}{\bar{k} b N_{1}} \text { и } \quad \Phi_{1}=C \frac{K_{0}^{2} a^{2} d_{0}^{6} D_{0}^{6}}{(\bar{k})^{3} b^{2} N_{1} \mathscr{L}} .
$$

С помошью леммы П.1, (П.21), получаем, что

$$
\left|I_{1}[u]\right| \leqslant \sum_{s_{c}^{1}} \frac{A_{c}[u]}{\left|\Omega S^{(2)}\left(s_{c}^{1}\right)\right|^{1 / 2}}+\frac{A[u, S]}{\Omega},
$$

и все величины в правой части этой оценки явно выражены через введенные параметры. Теперь нужная оценка интеграла $I_{1}^{ \pm}\left[\left(1-\chi_{n m}^{ \pm}\right) w_{n m}^{ \pm}\right]$получается из (5.29). Подставляя полученную оценку и (5.20) в (5.18), имеем

$$
\begin{aligned}
\left|J_{n m}\left[u_{n}\right]\right| & \leqslant \frac{2 \pi}{\Omega} \sum_{ \pm} \sum_{s_{c}^{1}} \frac{A_{c}\left[w_{n m}^{ \pm}\left(1-\chi_{n m}^{ \pm}\right)\right]}{\left|\Omega S_{ \pm}^{(2)}\left(s_{c}^{1}\right)\right|^{1 / 2}}+\Delta_{1} J_{n m}+\Delta J_{n m} \\
\left|\Delta_{1} J_{n m}\right| & \leqslant \frac{C N_{1}^{2}(\bar{k})^{3} b^{p+3} \mathscr{L}^{2} A(n)}{\Omega^{2} k_{D}^{3} d_{0}^{8} a^{2} D_{0}^{6}}+\frac{C \mathscr{L} a^{j_{0}} A(n)}{\Omega d_{0}}
\end{aligned}
$$

и, следовательно, $\Delta_{1} J_{n m} \in\{0\}$ при условиях

$$
8 \beta_{1}+6 \beta_{2}+3 \beta_{4}+2 \beta_{5}+C(p) \alpha \leqslant \frac{1}{2}-\varepsilon, \quad \beta_{5} j_{0} \geqslant \frac{1}{2}+\varepsilon+\beta_{4}+\beta_{1}+C \alpha .
$$


В частности, отсюда следует, что $\beta_{4}<\frac{1}{6}$, и из (5.13) получаем, что $\varkappa>10$. Это условие - наиболее сильное ограничение на скорость убывания $S^{A}$.

Пусть теперь $\vec{r}_{0} \in D_{\beta_{1} \beta_{2}}^{2},\left|\vec{r}_{0}-\vec{r}_{m}^{0}\right| \geqslant D^{\prime}$. Тогда достаточно рассмотреть случай $d_{j} \equiv d\left(\vec{r}_{0}, T\left(s_{j}^{0}\right)\right) \leqslant D_{0}$. Даже если касательные $T\left(s_{i}^{0}\right), T\left(s_{j}^{0}\right)$ пересекаются, вклады точек $s_{i}^{0}, s_{j}^{0}$ можно рассматривать по отдельности. При $\left|\vec{r}_{0}-\vec{r}_{m}^{0}\right| \leqslant D^{\prime}$ происходит простое суммирование вкладов точек $s_{i}^{0}$, отвечаюших прямым $T\left(s_{i}^{0}\right)$, пересекаюшимся в точке $\vec{r}_{m}^{0}(i \subset(m)(2.30))$. Поэтому случай $\left|\vec{r}_{0}-\vec{r}_{m}^{0}\right| \leqslant D^{\prime}$ можно отдельно не рассматривать. Чтобы его рассмотреть, достаточно в полученных при $\left|\vec{r}_{0}-\vec{r}_{m}^{0}\right| \geqslant D^{\prime}$ оценках заменить $\vec{r}_{0 j}$ на $\vec{r}_{m}^{0}$ и $D_{0}$ на $D^{\prime}$. Пусть $\vec{r}_{0} \in T_{D_{j}}$ и $j$ фиксировано. Для оценки интеграла $I_{1}[u](5.21)$ будем использовать лемму П.2, согласно которой все оценки производятся для точки $\vec{r}_{0 j}-$ проекции $\vec{r}_{0}$ на $T\left(s_{j}^{0}\right)$. Множество критических точек $s_{0 c} \equiv s_{c}^{1}\left(r_{0 j}\right)$ включает невырожденные критические точки $s_{0 c}^{1}$ и вырожденную $-s_{j}^{0}$. Так как невырожденные точки $s_{0 c}^{1}$ "далеки" от $s_{j}^{0}$ в смысле леммы П.3, то (см. (4.14), (4.15))

$$
\left|S^{(2)}\left(s_{0 c}^{1}\right)\right| \geqslant a\left|k\left(s_{0 c}^{1}\right)\right|, \quad\left|S^{\left(p_{j}+2\right)}\left(s_{j}^{0}\right)\right| \geqslant a K_{j}
$$

и для $k\left(s_{0 c}^{1}\right)$ выполняется оценка (5.26). Величины $\delta_{0 c}=\left(\delta_{0}, \delta_{j}\right), \Delta_{0 c}=\left(\Delta_{0}, \Delta_{j}\right), \Psi_{\Delta}$ в оценке (П.28) можно выбрать следуюшим образом:

$$
\begin{gathered}
\delta_{c}=\delta_{0}=C \frac{k_{d} d_{0}^{4} a}{\bar{k} b N_{1}}, \quad \delta_{j}=C \frac{d_{0}^{p_{j}+4} K_{j} a^{\frac{1}{p_{j}+1}}}{\bar{k} b^{p_{j}+1} N_{1}^{1 /\left(p_{j}+1\right)}}, \\
\Delta_{0}=C \frac{k_{d} d_{0}^{2}}{\bar{k} b}, \quad \Delta_{j}=C \frac{K_{j} d_{0}^{p_{j}+1}}{\bar{k} b^{p_{j}+1}}, \quad \Psi_{\Delta}=C a D^{\prime} .
\end{gathered}
$$

Это позволяет явно указать величину $\Phi_{01}$ (П.28) в оценке (П.27). Условие (П.25) выполняется, если

$$
\beta_{2} \geqslant C(p) \alpha+2 \beta_{5}+2 \beta_{4}, \quad \beta_{2} \geqslant \beta_{3}+\beta_{4}+\beta_{5}+2 \alpha .
$$

Используя оценку (П.26) при $u=\left(1-\chi_{n m}^{ \pm}\right) u_{n}$ и подставляя результат в (5.18), получим при $\vec{r}_{0} \in D_{\beta_{1} \beta_{2}}^{2}, d_{j} \leqslant D_{0}$ (с учетом $\left.(5.20)\right)$

$$
\begin{aligned}
\left|J_{n m}\left[u_{n}\right]\right| \leqslant \frac{2 \pi}{\Omega} \sum_{ \pm}\left[\sum_{s_{c}^{1}\left(\vec{r}_{0 j}\right)} \frac{A_{0 c}\left[w_{n m}^{ \pm}\left(1-\chi_{n m}^{ \pm}\right)\right]}{\left|\Omega S_{ \pm}^{(2)}\left(s_{0 c}^{1}, r_{0 j}\right)\right|^{1 / 2}}\right. & \\
& \left.\quad \frac{A_{j}\left[w_{n m}^{ \pm}\left(1-\chi_{n m}^{ \pm}\right)\right]}{\left|\Omega S_{ \pm}^{\left(p_{j}+2\right)}\left(s_{j}^{0}\right)\right|^{1 /\left(p_{j}+2\right)}}\right]+\Delta_{1} J_{n m}+\Delta J_{n m}
\end{aligned}
$$

В этой формуле $A_{j}[u] \equiv A_{0 c}[u]\left(s_{0 c}=s_{j}^{0}\right)$, функционалы $A_{0 c}[u]$ оценены в (П.22) и $\Delta_{1} J_{n m} \in\{0\}$ при условиях

$$
C(p) \beta_{1}+3 \beta_{4}+C(p) \alpha \leqslant \frac{1}{2}-\varepsilon, \quad \beta_{5} j_{0} \geqslant \frac{1}{2}+\varepsilon+\beta_{4}+C \alpha+\beta_{1} .
$$

Таким образом, для доказательства леммы 5.1 достаточно получить удовлетворительную оценку величины $\Delta J_{n m}$ в (5.18). Для этого рассмотрим по отдельности интегралы трех типов: $J_{n m}\left[\rho_{t} u_{n}\right]\left(\rho_{t}=\widetilde{\rho}, \rho_{0}, \rho_{i}\right)$. 
5.2.2. Интеграл $J_{n m}\left[\widetilde{\rho} u_{n}\right]$. Везде ниже используется обозначение

$$
J_{n m}^{2}\left[u_{n}\right]=\int_{0}^{B} d \xi \int_{0}^{2 \pi} e^{i \Omega \Phi_{n m}} u_{n} d \varphi
$$

В этом интеграле $s$ играет роль параметра и зависимости различных величин от $s$ явно не указьваются.

Рассмотрим двумерньй интеграл $J_{n m}^{2}\left[\widetilde{\rho} u_{n}\right]$, входящий в выражение

$$
J_{n m}\left[\widetilde{\rho} u_{n}\right]=\int_{\partial \mathscr{D}} d s J_{n m}^{2}\left[\widetilde{\rho} u_{n}\right]
$$

Taк как

$$
\begin{gathered}
\Phi_{n m}(\varphi, \xi)=f(\varphi)+\xi g(\varphi), \\
f(\varphi)=-2 L m \varphi+2 n\langle\vec{r}, \vec{\eta}\rangle, \quad g(\varphi)=\langle\vec{l}, \vec{\eta}\rangle,
\end{gathered}
$$

то $J_{n m}^{2}\left[\widetilde{\rho} u_{n}\right]$ - специальньй двумерный интеграл, рассмотренньй в приложении. Критические точки $\left(\varphi_{c}, \xi_{c}\right)=\left(\varphi_{ \pm}, \xi_{ \pm}\right)$фазы $\Phi_{n m}$ как функции от переменных $\varphi, \xi$ проанализированы в $\S 4$, где показано, что $g^{(1)}\left(\varphi_{c}\right)=l, g^{(2)}\left(\varphi_{c}\right)=0$. Таким образом, можно использовать теорему П.2, и в интересуюшем нас случае (см. (3.4))

$$
\lambda=N_{1}, \quad \lambda_{1}=\lambda_{2}=1, \quad g_{1}=\tilde{g}=\bar{g}=l \geqslant d_{0}, \quad v_{|\alpha|}=A(n) .
$$

Условия (П.31), (П.32) имеют вид

$$
N_{1}^{5} d_{0}^{-6} \leqslant C \Omega^{1-\varepsilon_{2}}, \quad N_{1}^{3} d_{0}^{-4} a^{-2} \leqslant C \Omega^{1-\varepsilon_{1}}, \quad \varepsilon_{2}>\varepsilon_{1},
$$

и они вьполняются, если

$$
5 \beta_{4}+6 \beta_{1} \leqslant 1-\varepsilon_{2}, \quad 3 \beta_{4}+4 \beta_{1}+2 \beta_{5} \leqslant 1-\varepsilon_{1} .
$$

В случае $\vec{r}_{0} \in D_{\beta_{1} \beta_{2}}^{1} \cup D_{\beta_{1} \beta_{2}}^{2}$ достаточно в (П.33) взять $Q=0, T=2$, чтобы получить

$$
J_{n m}\left[\widetilde{\rho} u_{n}\right]=\frac{2 \pi}{\Omega} \sum_{ \pm} I_{1}^{ \pm}\left[\widetilde{\rho} u_{n}\right]+\Delta_{2} J_{n m}
$$

вместе с оценкой

$$
\left|\Delta_{2} J_{n m}\right| \leqslant \frac{C\left(\varepsilon_{1}, \varepsilon_{2}\right) \mathscr{L} A(n) N_{1}}{\Omega^{2} a^{2} d_{0}^{3}}
$$

где величина $A(n)$ определена в (3.4). 
5.2.3. Интеграл $J_{n m}\left[\rho_{0} u_{n}\right]$. Начнем с рассмотрения двух специальных случаев, когда этот интеграл легко оценивается.

Пусть $n=0, m \neq 0$; рассмотрим интеграл $J_{0 m}^{2}\left[\rho_{0} u_{n}\right]$ (5.36). При $n=0\left|\xi_{n m}^{ \pm}\right| \geqslant 2 L$ и, следовательно, если $L \geqslant 2 a_{0}$, то для этого интеграла в носителе амплитуды $\rho_{0} u_{0}$ нет критических точек. Используем оценку

$$
\left|J_{0 m}\left[\rho_{0} u\right]\right| \leqslant \mathscr{L} \max _{s}\left|J_{0 m}^{2}\left[\rho_{0} u_{0}\right]\right| .
$$

Интеграл $J_{0 m}^{2}\left[\rho_{0} u_{0}\right]$ оценивается с помошью (П.2), (П.4). При вьполнении условий

$$
\alpha+\beta_{6} \leqslant 1-\varepsilon^{\prime}, \quad 4 \alpha \leqslant 1-\varepsilon^{\prime}, \quad \beta_{6}>\alpha
$$

для всех $T, \varepsilon^{\prime}>0$ имеет место оценка

$$
\left|J_{0 m}\left[\rho_{0} u_{0}\right]\right| \leqslant C_{T}\left(\varepsilon^{\prime}\right) A(n) \mathscr{L} \Omega^{-T} .
$$

Рассмотрим теперь случай $L|m| \geqslant 4|n|, n \neq 0$. Интеграл $J_{n m}\left[\rho_{0} u_{n}\right]$ запишем в виде

$$
\begin{aligned}
J_{n m}\left[\rho_{0} u_{n}\right] & =\int_{0}^{\infty} d \xi \int_{\partial \mathscr{D}} d s \rho_{0}(\xi) v_{n}(\xi) Y_{n m}^{1} \\
Y_{n m}^{1} & =\int_{0}^{2 \pi} e^{i \Omega \Phi_{n m}}[\vec{\tau}, \vec{\eta}] d \varphi
\end{aligned}
$$

Так как $\left|\partial_{\varphi} \Phi_{n m}\right| \geqslant C L|m|$ при $L|m| \geqslant 4|n|$, то интегрируя в $Y_{n m}^{1}$ по частям, с помошью (П.2), (П.4) и используя оценку

$$
\left|J_{n m}\left[\rho_{0} u_{n}\right]\right| \leqslant a_{0} \mathscr{L} \max _{\xi, s}\left|Y_{n m}^{1}\right|
$$

получим, что при $L|m| \geqslant 4|n|$ и тех же условиях (5.42)

$$
\left|J_{n m}\left[\rho_{0} u_{n}\right]\right| \leqslant C_{T}\left(\varepsilon^{\prime}\right) A(n) \mathscr{L} \Omega^{-T} \quad \forall T, \varepsilon^{\prime}>0 .
$$

Обе оценки (5.43), (5.44) равномерны по $\vec{r}_{0}$ и не зависят от предположения, что $\vec{r}_{0} \in$ $D_{\beta_{1} \beta_{2}}^{1} \cup D_{\beta_{1} \beta_{2}}^{2}$.

Осталось рассмотреть случай $L|m| \leqslant C|n|, n \neq 0$. В этом случае используется представление

$$
J_{n m}\left[\rho_{0} u_{n}\right]=\int_{\partial \mathscr{D}} d s J_{n m}^{2}\left[\rho_{0} u_{n}\right]
$$

и для исследования интеграла $J_{n m}^{2}\left[\rho_{0} u_{n}\right](5.36)$ применяется теорема П.4, в которой

$$
\begin{gathered}
n_{0}=1, \quad \lambda=C|n|, \quad \lambda_{1}=\lambda_{2}=1, \quad g_{1}=\widetilde{g}=\bar{g}=l, \\
t_{0}=C \Omega^{-\frac{1-\varepsilon_{1}}{2}}|n|^{5 / 2} l^{-3}, \quad v_{|\alpha|}=A(n) .
\end{gathered}
$$


Условия теоремы П.4 будут выполнены, если к условиям (5.39) добавить требование

$$
a_{0} \geqslant C \Omega^{-\frac{\varepsilon_{2}-\varepsilon_{1}}{6}} d_{0}^{-3} \quad\left(\sigma=\frac{\varepsilon_{2}-\varepsilon_{1}}{3} \text { в }(\Pi .54)\right)
$$

которое вьполняется, если

$$
\beta_{6}+3 \beta_{1}<\frac{\varepsilon_{2}-\varepsilon_{1}}{6}
$$

При $J_{2}[u] \equiv J_{n m}^{2}\left[\rho_{0} u_{n}\right]$ в $(\Pi .44)$ граничньй член $\left(J_{n m}^{2}\right)_{0}^{P}$ оценивается с помошю (П.50). Выбирая в (П.44) $Q=0, T=2$ и подставляя результат в (5.45), имеем

$$
\begin{aligned}
J_{n m}\left[\rho_{0} u_{n}\right]= & \frac{2 \pi}{\Omega} \sum_{ \pm} I_{1}^{ \pm}\left[\rho_{0} w_{n m}^{ \pm}\right]-\frac{2 \pi}{\Omega} \sum_{( \pm) \subset(1)} I_{1}^{ \pm}\left[\rho_{0} w_{n m}^{ \pm}\right] \\
& +\frac{2 \pi}{\Omega} \sum_{( \pm) \subset(1)} I_{1}^{ \pm}\left[w_{n m}^{ \pm, 1}\right]+\Delta_{3}^{1} J_{n m} .
\end{aligned}
$$

В этой формуле запись $( \pm) \subset(1)$ означает, что $\left|\xi_{n m}^{ \pm}\right| \leqslant C t_{0}$. В обозначениях теоремы П.3 условиям $( \pm) \subset(1)$ отвечают критические точки $\left(\varphi_{c 1}, \xi_{c 1}\right)$ и функция $w_{n m}^{ \pm, 1}$ в (5.47) определяется равенством

$$
w_{n m}^{ \pm, 1}(s)=\sum_{k=1}^{N-1} \frac{\partial_{\xi}^{k} u_{n}\left(\varphi_{+}, 0\right)}{k ! l}\left(\xi_{n m}^{ \pm}(s)\right)^{k}\left[\mathscr{K}_{ \pm}-i \pi\right]
$$

где $N=N\left(\varepsilon_{1}, \varepsilon_{2}\right)$ и $\mathscr{K}_{ \pm} \equiv \mathscr{K}_{c 1}(\Pi .45)$. Используя $(\Pi .76)$, получим, что

$$
\begin{gathered}
w_{n m}^{ \pm, 1}(s)=w_{n m}^{ \pm, 2}(s)+w_{n m}^{ \pm, 3}(s), \\
w_{n m}^{ \pm, 2}(s)=i \pi \sum_{k=1}^{N-1} \frac{\partial_{\xi}^{k} u_{n}\left(\varphi_{+}, 0\right)}{k ! l(s)}\left(\xi_{n m}^{ \pm}(s)\right)^{k}\left(1+\operatorname{sgn} \xi_{n m}^{ \pm}(s)\right), \\
w_{n m}^{ \pm, 3}(s)=\sum_{k=1}^{N-1} \frac{\partial_{\xi}^{k} u_{n}\left(\varphi_{ \pm}, 0\right)}{k ! l(s)}\left(\xi_{n m}^{ \pm}(s)\right)^{k} \Delta \mathscr{K}_{ \pm}
\end{gathered}
$$

и, соответственно,

$$
I_{1}^{ \pm}\left[\rho_{0}, w_{n m}^{ \pm, 1}\right]=I_{1}^{ \pm}\left[\rho_{0} w_{n m}^{ \pm, 2}\right]+I_{1}^{ \pm}\left[\rho_{0} w_{n m}^{ \pm, 3}\right] .
$$

Прямо из (П.50), (П.55) при $\varepsilon_{1}<\frac{1}{2}$ следует оценка последнего члена в (5.47):

$$
\left|\Delta_{3}^{1} J_{n m}\right| \leqslant C\left(\varepsilon_{1} \varepsilon_{2}\right) \mathscr{L} A(n)\left[\frac{(\ln \Omega)|n|}{\Omega^{2} a_{0}^{6} d_{0}^{3}}+\frac{1}{a_{0}|n|^{\frac{3}{2}}+\frac{\varepsilon_{1}}{2} \Omega^{\frac{3}{2}+\frac{\varepsilon_{1}}{2}}}+\frac{|n|^{5}}{a_{0}^{3} d_{0}^{6} \Omega^{2-\varepsilon_{1}}}\right] .
$$

Оценка второго и третьего членов в правой части (5.47) сводится к оценке интегралов $I_{1}^{ \pm}\left[\rho_{0} w_{n m}^{ \pm}\right], I_{1}^{ \pm}\left[\rho_{0} w_{n m}^{ \pm, i}\right] \quad(i=1,2)$, амплитуда в которых мала, так как $\left|\xi_{n m}^{ \pm}(s)\right| \leqslant t_{0}$. Первьй и второй из этих интегралов оценивается так же, как интеграл $I_{1}[u]$ в п. 5.2.1. 
Рассмотрим оценку интеграла $I_{n m}^{ \pm}\left[\rho_{0} w_{n m}^{ \pm, 3}\right]$. В этом случае трудности связаны с тем, что хотя амплитуда и мала, $\left|w_{n m}^{ \pm, 3}\right| \leqslant C(\ln \Omega) t_{0}$, но она может быстро осциллировать. Оценка этого интеграла основана на результатах, изложенных в п. 6 приложения. В интересуюшем нас случае (см. (П.51), (П.72)) $b_{1}=-\xi_{n m}^{ \pm}(s), b_{2}=-n\langle\vec{l}, \vec{r}\rangle \times$ $(l(s))^{-2}, y_{0}=C(l(s))^{-1}|n|^{3 / 2} \Omega^{-\frac{1-\varepsilon_{1}}{2}}$.

Разделим область интегрирования $U$ в интеграле $I_{1}^{ \pm}\left[\rho_{0} w_{n m}^{ \pm, 3}\right]$ на две области $U=U_{1} \cup U_{2}$, в которых соответственно $s \in U_{1} \Leftrightarrow\left|b_{2}\right| \leqslant C \Omega^{-\varepsilon_{1}+2 \varepsilon}|n|^{5} d_{0}^{-6}$ и $s \in U_{2} \Leftrightarrow C \Omega^{-\varepsilon_{1}+2 \varepsilon}|n|^{5} d_{0}^{-6}<\left|b_{2}\right|$. Тогда $I_{1}^{ \pm}\left[\rho_{0} w_{n m}^{ \pm, 3}\right]=I_{11}+I_{12}$. Интеграл $I_{11}$ по области $U_{1}$ оценивается тривиально: $\left|I_{11}\right| \leqslant C \mathscr{L} t_{0} A(n)\left|\Delta \mathscr{K}_{ \pm}\right|$, и для $\left|\Delta \mathscr{K}_{ \pm}\right|$ используется оценка (П.80). В области $U_{2} \Omega\left|b_{2}\right| y_{0}^{2}>C \Omega^{2 \varepsilon}$, и для оценки интеграла $I_{12}$ используется лемма П.5. При этом возникает ОИ $Y_{ \pm}$с фазой $F_{ \pm}$ $\left(F_{ \pm}(s)=S_{ \pm}(s)+b_{1}^{2} /\left(4\left|b_{2}\right|\right)\right)$, и критические точки этой фазы близки к $s_{c}^{1}$. Область интегрирования $U_{2}$ снова разбиваем на две области: $U_{2}=U_{2}^{1} \cup U_{2}^{2}$, в которых, соответственно, $\left|s-s_{c}^{1}\right|<\delta$ и $\left|s-s_{c}^{1}\right|>\delta$ для всех $s_{c}^{1}$. Интеграл $Y_{ \pm}^{1}$ по области $U_{2}^{1}$ оценивается тривиально: $\left|Y_{ \pm}^{1}\right| \leqslant C A(n) t_{0} \delta \mathscr{N}_{1}$, а интеграл $Y_{ \pm}^{2}$ по области $U_{2}^{2}$, где нет критических точек, оценивается с помошю интегрирования по частям. Выбирая подходящим образом величину $\delta$, получим оценку $Y_{ \pm}$, а следовательно, и оценку $I_{1}^{ \pm}\left[\rho_{0} w_{n m}^{ \pm, 3}\right]$. Собирая все оценки, имеем

$$
J_{n m}\left[\rho_{0} u_{n}\right]=\frac{2 \pi}{\Omega} \sum_{ \pm} I_{1}^{ \pm}\left[\rho_{0} w_{n m}^{ \pm}\right]+\Delta_{3} J_{n m}
$$

и при этом

$$
\left|\Delta_{3} J_{n m}\right| \leqslant\left|\Delta_{3}^{1} J_{n m}\right|+\left|\Delta_{3}^{2} J_{n m}\right|,
$$

где величина $\Delta_{3}^{1} J_{n m}$ оценена в $(5.48)$ и

$$
\left|\Delta_{3}^{2} J_{n m}\right| \leqslant \frac{C}{\Omega} \begin{cases}\Delta I_{1}\left(k_{D}\right) & \left(\vec{r}_{0} \in D_{\beta_{1} \beta_{2}}^{1}\right), \\ \Delta I_{1}^{1} & \left(\vec{r}_{0} \in D_{\beta_{1} \beta_{2}}^{2}\right) .\end{cases}
$$

В этой формуле

$$
\begin{aligned}
\Delta I_{1}\left(k_{D}\right)= & C_{N}(\varepsilon) A(n)\left[\frac{\mathscr{L}|n|^{8}}{\Omega^{1-2 \varepsilon_{1}} d_{0}^{10}}+\frac{\mathscr{L}^{2}(\bar{k})^{3} b^{2} \mathscr{N}_{1}}{k_{D}^{4} d_{0}^{9} D_{0}^{2} \Omega^{1-\frac{7 \varepsilon_{1}}{2}}}+\frac{|n|^{\frac{5}{2}} \mathscr{N}_{1}}{\Omega^{\frac{1}{2}+\frac{\varepsilon_{1}}{2}-\varepsilon} d_{0}^{3}}\right], \\
\Delta I_{1}^{1}= & \Delta I_{1}\left(k_{d}\right)+C_{N}(\varepsilon) A(n) \\
& \times\left[\frac{\mathscr{N}_{1}|\bar{k}|^{3(p+1)} b^{2(p+1)^{2}}}{\left(K_{0}\right)^{3 p+4} d_{0}^{(p+1)(p+8)+1} \Omega^{1-\varepsilon_{1}\left(p+\frac{5}{2}\right)}}+\frac{\mathscr{N}_{1} \mathscr{L}^{2}}{d_{0}^{2}\left(D^{\prime}\right)^{2} \Omega^{1-\frac{7 \varepsilon_{1}}{2}}}\right] .
\end{aligned}
$$

Оценка (5.52) справедлива при условии

$$
8 \beta_{1}+6 \beta_{2}+C \alpha<\frac{1}{2}-\frac{5 \varepsilon_{1}}{2}
$$


оценка (5.53) - при условиях

$$
\begin{gathered}
\varepsilon_{1}<1 / 4, \quad \beta_{3}<\frac{1}{2}-\frac{3 \varepsilon_{1}}{2}, \quad \beta_{2}>\beta_{3}+\alpha, \\
C_{1}(p) \beta_{1}+C_{2}(p) \alpha \leqslant \frac{1}{2}-\frac{3 \varepsilon_{1}}{2}+\varepsilon_{1} p, \quad \beta_{2} \geqslant C_{2}(p) \beta_{1}+C_{1}(p) \alpha .
\end{gathered}
$$

Как уже указьвалось, для оценки интегралов $I_{1}^{ \pm}\left[\rho_{0} w_{n m}^{ \pm}\right], I_{1}^{ \pm}\left[\rho_{0} w_{n m}^{ \pm, 2}\right]$ можно воспользоваться результатами, полученньми в п. 5.2.1 при оценке интегралов вида $I_{1}[u]$. Учитывая, что нас интересует случай $( \pm) \subset(1)$, получим, что

$$
\left|I_{1}^{ \pm}\left[\rho_{0} w_{n m}^{ \pm, 2}\right]\right| \leqslant\left\{\begin{array}{l}
\Delta I_{1}\left(k_{D}\right), \\
\Delta I_{1}^{1} .
\end{array}\right.
$$

Теперь заметим, что $I_{1}^{ \pm}\left[\rho_{0} w_{n m}^{ \pm}\right]=0$, если $n=0$ или $L|m| \geqslant 4|n|$. Выбирая в оценках (5.43), (5.44) величину $T$ нужным образом, получим, что оценки $(5.50),(5.48)$, (5.51) верны во всей области $(n, m) \neq(0,0)$.

5.2.4. Интеграл $J_{n m}\left[\rho_{i} u_{n}\right]$. Снова используем представление

$$
J_{n m}\left[\rho_{i} u_{n}\right]=\int_{\partial \mathscr{D}} d s J_{n m}^{2}\left[\rho_{i} u_{n}\right] .
$$

$\Phi$ азу $\Phi_{n m}$ в интеграле $J_{n m}^{2}\left[\rho_{i} u_{n}\right]$ запишем в виде

$$
\begin{gathered}
\Phi_{n m}(\varphi, \xi)=f_{i}(\varphi)+\left(\xi-\xi_{i}^{n}\right) g(\varphi), \\
f_{i}(\varphi)=-2 L m \varphi+\left\langle\xi_{i}^{n} \vec{l}+2 n \vec{r}, \vec{\eta}\right\rangle, \quad g(\varphi)=\langle\vec{l}, \vec{\eta}\rangle .
\end{gathered}
$$

Таким образом, $J_{n m}^{2}\left[\rho_{i} u_{n}\right] \in\left\{\widetilde{J}_{2}\right\}$ и можно использовать следствие П.1 теоремы П.5. При этом $n_{0}=j_{1}, n_{1}=1$, выполняется $(5.38)$ и $t_{0}=C \Omega^{-\frac{1-\varepsilon_{1}}{2}} N_{1}^{\frac{5}{2}} d_{0}^{-3}$. Выбирая в (П.65) $Q=0, T=3$, получим

$$
J_{n m}^{2}\left[\rho_{i} u_{n}\right]=\sum_{ \pm}\left(J_{n m}^{2}\right)_{ \pm}^{0}+\Delta J_{n m}^{2}\left[\rho_{i} u_{n}\right]
$$

Первый член в правой части этой формулы - главный критический вклад точек $\left(\varphi_{ \pm}, \xi_{ \pm}\right)(\Pi .15)$, и в соответствии с $(\Pi .65)$

$$
\begin{aligned}
\Delta J_{n m}^{2}\left[\rho_{i} u_{n}\right]= & \left(J_{n m}^{2}\right)_{0}^{P}+\Delta_{03}\left(J_{n m}^{2}\left[\rho_{i} u_{n}\right]\right)+\Delta^{1}\left(J_{n m}^{2}\left[\rho_{i} u_{n}\right]\right) \\
& +i \Omega^{-1} \sum_{( \pm) \subset(1)} e^{i \Omega f_{i}\left(\varphi_{ \pm}\right)} \mathscr{K}_{ \pm} T_{ \pm}^{0} .
\end{aligned}
$$

Три первых члена в правой части этого выражения оценены соответственно в (П.50), (П.36), (П.66), а величина $T_{ \pm}^{0}$ определена в (П.64), и из этого равенства сразу следует оценка $T_{ \pm}^{0}$, так как $\left|b_{1}\right| \leqslant t_{0}$. Из (П.52) получаем, что $\left|\mathscr{K}_{ \pm}\right| \leqslant C \ln \Omega$. Выбираем 
согласно (П.54) $\sigma=\frac{1}{3}\left(\varepsilon_{2}-\varepsilon_{1}\right) \quad\left(p=\frac{1}{6}\left(\varepsilon_{2}-\varepsilon_{1}\right)\right)$. Тогда условия следствия П.1 выполняются, если, кроме (5.39), вьполнено неравенство

$$
3 \beta_{1}+\beta_{5} \leqslant \frac{1}{6}\left(\varepsilon_{2}-\varepsilon_{1}\right) \quad\left(a>C \Omega^{-\frac{\varepsilon_{2}-\varepsilon_{1}}{6}} d_{0}^{-3}\right) .
$$

Подставляя $(5.56)$ в исходное представление для $J_{n m}\left[\rho_{i} u_{n}\right]$, получим

$$
\sum_{\xi_{c}^{n}} J_{n m}\left[\rho_{i} u_{n}\right]=\frac{2 \pi}{\Omega} \sum_{\xi_{i}^{n}} I_{1}^{ \pm}\left[\rho_{i} u_{n}\right]+\Delta_{4} J_{n m} .
$$

Так как $\Delta \xi \geqslant C \simeq 1(2.4)$, то, используя полученные оценки, имеем

$$
\begin{aligned}
\left|\Delta_{4} J_{n m}\right| & \leqslant \frac{\mathscr{L} N_{1}}{\Delta \xi} \max _{s, i}\left|\Delta J_{n m}^{2}\left[\rho_{i} u_{n}\right]\right| \\
& \leqslant C\left(\varepsilon_{1}, \varepsilon_{2}\right) \mathscr{L} A(n) N_{1}\left[\frac{N_{1}}{\Omega^{2} d_{0}^{2} a^{6}}+\ln \Omega\left(\frac{N_{1}^{5 / 2}}{\Omega^{\frac{1-\varepsilon_{1}}{2}} d_{0}^{3} a_{0}^{2}}\right)^{j_{1}}+\frac{1}{\Omega^{\frac{\varepsilon_{2}-\varepsilon_{1}}{3} j_{1}}}\right] .
\end{aligned}
$$

5.2.5. Завершение доказательства леммы 5.1. Складьвая (5.40), (5.49), (5.58) и учитывая (5.14), получим равенство (5.18), в котором

$$
\left|\Delta J_{n m}\right| \leqslant \sum_{k=2}^{5}\left|\Delta_{k} J_{n m}\right|, \quad\left|\Delta_{5} J_{n m}\right| \leqslant C \mathscr{L} A(n) \Omega^{-\beta_{4} \varkappa},
$$

а величины $\Delta_{k} J_{n m}(k=2,3,4)$ оценены в $(5.41),(5.50),(5.59)$. В оценки $(5.34),(5.30)$ входит еще и величина $\Delta_{1} J_{n m}$, и $\Delta_{1} J_{n m} \in\{O\}$ при условиях (5.35), (5.31).

Пусть $\vec{r}_{0} \in D_{\beta_{1} \beta_{2}}^{1}$. Рассмотрим условия, при которых $\Delta_{k} J_{n m} \in\{O\}(k=1, \ldots, 5)$. Полученные оценки $\Delta_{k} J_{n m}$ имеют вид $\left|\Delta_{k} J_{n m}\right| \leqslant \Delta_{k}^{1} J_{n m}+\Delta_{k}^{2} J_{n m}$, где величины $\Delta_{k}^{1} J_{n m}$ не зависят от гладкости алгоритма, т.е. от $\varkappa, j_{0}, j_{1}$, а величины $\Delta_{k}^{2} J_{n m}-$ зависят. Из (5.9) и (5.59) следует, что $\Delta_{4}^{2} J_{n m} \in\{O\}$, если

$$
\begin{aligned}
& j_{1} \frac{\varepsilon_{2}-\varepsilon_{1}}{6} \geqslant \frac{3}{2}+\varepsilon+2 \beta_{4}+C \alpha \\
& j_{1}\left(\frac{1}{2}-\frac{\varepsilon_{1}}{2}-\frac{5 \beta_{4}}{2}-2 \beta_{5}+3 \beta_{1}\right) \geqslant \frac{3}{2}+\varepsilon+2 \beta_{4}+C \alpha .
\end{aligned}
$$

Таким образом, $\Delta_{k}^{2} J_{n m} \in\{O\}, \Delta_{F} \mu^{A} \in\{O\}$ при условиях (5.13), (5.31), (5.60). Рассмотрим величины $\Delta_{k}^{1} J_{n m}(k=1, \ldots, 4)$. Они имеют вид

$$
\begin{aligned}
& \Delta_{k}^{1} J_{n m}=\sum_{p=1}^{p(k)} C_{k} \frac{A(n) Q_{k p}(\ln \Omega)}{\Omega^{3 / 2+\gamma_{k p}}} \quad\left(\gamma_{k p}>0\right), \\
& Q_{k p}=\frac{N_{1}^{x_{4}}}{d_{0}^{x_{1}} D_{0}^{x_{2}} a^{x_{3}}} \cdot \frac{t_{1}^{y_{1}} \ldots t_{4}^{y_{4}}}{t_{5}^{y_{5}} \ldots t_{8}^{y_{8}}} \quad\left(y_{i} \equiv y_{k, p, i}, x_{i}=x_{k, p, i}\right) \text {. }
\end{aligned}
$$


Напомним, что $t_{1}, \ldots, t_{4}$ - большие, a $t_{5}, \ldots, t_{8}$ - малые параметры (см. (2.17)).

Используя (5.9), запишем условия, при которых $\Delta_{k}^{1} J_{n m} \in\{O\}$, в виде ${ }^{2}$

$$
\sum_{i=1}^{5} C_{k p}^{i} \beta_{i}+C_{k p} \alpha \leqslant \gamma_{k p}-\varepsilon \quad(p=1, \ldots, p(k), k=1, \ldots, 4) .
$$

Из приведенных выше условий извлекается явный вид величин $\gamma_{k p}$ и констант $C_{k p}^{i}, C_{k p}$. В частности, из этих условий следует, что $\gamma_{1 p}=\gamma_{2 p}=\gamma_{4 p}=\frac{1}{2}$,

$$
\begin{array}{ll}
\min _{p} \gamma_{3 p}=\min \left\{\frac{\varepsilon_{1}}{2}, \frac{1}{2}-\frac{7 \varepsilon_{1}}{2}\right\} & \left(\vec{r}_{0} \in D_{\beta_{1} \beta_{2}}^{1}\right), \\
\min _{p} \gamma_{3 p}=\min \left\{\frac{\varepsilon_{1}}{2}, \frac{1}{2}-\left(\frac{5}{2}+p\right) \varepsilon_{1}\right\} & \left(\vec{r}_{0} \in D_{\beta_{1} \beta_{2}}^{2}\right) .
\end{array}
$$

При $\vec{r}_{0} \in D_{\beta_{1} \beta_{2}}^{1}$, подставляя $(5.12),(5.30)$ в $(5.2)$, получим с учетом $(5.26),(5.12)$

$$
\left|\Delta \mu_{T}^{A}\left(\vec{r}_{0}\right)\right| \leqslant C \sum_{n, m}^{F} \sum_{ \pm} \sum_{s_{c}^{1}} \frac{A_{c}\left[\left(1-\chi_{n m}^{ \pm}\right) w_{n m}^{ \pm}\right]}{\left|\Omega S_{ \pm}^{(2)}\left(s_{c}^{1}\right)\right|^{1 / 2}}+R,
$$

где суммирование ведется по всем $n, m, \pm, s_{c}^{1}$ таким, что

$$
\begin{gathered}
(m, n) \neq(0,0), \quad|n| \leqslant N_{1}=C \Omega^{\beta_{4}}, \quad|m| \leqslant M=C \gamma N_{1} L^{-1}, \\
\left|\xi_{n m}^{ \pm}\left(s_{c}^{1}\right)+2 n\right|>a, \quad \xi_{n m}^{ \pm}\left(s_{c}^{1}\right) \geqslant 0 \quad\left(a=C \Omega^{-\beta_{5}}\right),
\end{gathered}
$$

и функционал $A_{c}[u]$ оценен в (П.22), а $R-$ в (2.28). Для функционала $A_{c}$ в (5.61) имеет место оценка

$$
A_{c}\left[\left(1-\chi_{n m}^{ \pm}\right) w_{n m}^{ \pm}\right] \leqslant\left|w_{n m}^{ \pm}\left(s_{c}^{1}\left(\vec{r}_{0}\right), \vec{r}_{0}\right)\right|+3 \delta_{c} \overline{\left(w_{n m}^{ \pm}\right)_{c}^{1}}+A(n) d_{0}^{-1} a^{j_{0}},
$$

и для завершения доказательства п. 1 леммы 5.1 достаточно показать, что

$$
\begin{aligned}
& R_{1}+R_{2} \leqslant C R, \\
& R_{1}=\sum_{n, m}^{F} \sum_{ \pm} \sum_{s_{c}^{1}} \frac{\delta_{c} \overline{\left(w_{n m}^{ \pm}\right)_{c}^{1}}}{\Omega S_{ \pm}^{(2)}\left(s_{c}^{1}\right)}, \quad R_{2}=\sum_{n, m}^{F} \sum_{ \pm} \sum_{s_{c}^{1}} \frac{A(n) a^{j_{0}}}{d_{0}\left|\Omega S_{ \pm}^{(2)}\left(s_{c}^{1}\right)\right|^{1 / 2}}
\end{aligned}
$$

и для $R$ верна оценка (2.28). Так как из (5.23), (5.25) следует оценка

$$
R_{2} \leqslant C \frac{\mathscr{L} \bar{k} b^{p+1} a^{j_{0}-\frac{1}{2}} N_{1}}{\Omega^{1 / 2} K_{0}^{5 / 4} D_{0}^{1 / 2} d_{0} L},
$$

\footnotetext{
2 Это условия (5.27), (5.31), (5.33), (5.35), (5.39), (5.42), (5.46), (5.54), (5.55), (5.57).
} 
то $R_{2} \leqslant C \Omega^{-\frac{1}{2}-\varepsilon}$, если

$$
\left(j_{0}-\frac{1}{2}\right) \beta_{5} \geqslant C(p) \alpha+\beta_{1}+\frac{1}{2} \beta_{2}+\varepsilon .
$$

Используя оценку

$$
\left|\frac{d}{d s} \xi_{n m}^{ \pm}(s)\right| \leqslant 2(L|m|+|n|) d_{0}^{-2},
$$

получим, что $\left|\left(w_{n m}^{ \pm}\right)^{(1)}(s)\right| \leqslant C\left(\bar{v}_{n}+\bar{v}_{n}^{(1)}\right) \frac{L|m|+|n|}{d_{0}^{3}}$. Величина $\delta_{c}$ определенав (5.28), и, таким образом, используя черту сверху для обозначения верхней оченки, получаем

$$
R_{1} \leqslant \frac{C \mathscr{L} b^{p} \bar{k} k_{D}^{1 / 2} a^{1 / 2} d_{0}}{\Omega^{1 / 2} N_{1}} \sum_{ \pm} \sum_{n, m}\left(\overline{v_{n}\left(\xi_{n m}^{ \pm}\right)}+\overline{v^{(1)}\left(\xi_{n m}^{ \pm}\right)}\right)(L|m|+|n|) .
$$

Непосредственно из определения величин $\xi_{n m}^{ \pm}$(4.2) получаем при $A \in\{A\}$ (см. (3.4))

$$
\left.\sum_{n, m}^{F} \overline{\left(v_{n}\left(\xi_{n m}^{ \pm}\right)\right.}+\overline{v^{(1)}\left(\xi_{n m}^{ \pm}\right)}\right)(L|m|+|n|) \leqslant C A_{0} L^{-\varkappa}
$$

и, следовательно,

$$
R_{1} \leqslant \frac{C \mathscr{L}\left(k_{D}\right)^{1 / 2} \bar{k} a^{1 / 2} d_{0}}{\Omega^{1 / 2} N_{1}} \cdot \frac{A_{0}}{L^{\varkappa}} .
$$

Условие $R_{1} \leqslant C \Omega^{-\frac{1}{2}-\varepsilon}$ выполняется, если

$$
\beta_{4}+\frac{1}{2} \beta_{5}+\frac{1}{2} \beta_{2} \geqslant \varepsilon+C(\varkappa, p) \alpha .
$$

Нужная оценка (5.15) теперь следует из (5.61) и остается убедиться в непротиворечивости условий на $\alpha, \beta_{i}$, которые были сформулированы выше. Выделим среди них условия, зависяшие от гладкости, т.е. включающие величины $j_{0}, j_{1}, \varkappa$. Все такие условия выполняются при достаточно больших $j_{0}, j_{1}, \varkappa$, если

$$
\beta_{5}>0, \quad \varepsilon_{2}>\varepsilon_{1}, \quad 1>\varepsilon_{1}+5 \beta_{4}+4 \beta_{5}+6 \beta_{1} .
$$

Остальные условия не зависят от гладкости и имеют вид

$$
\sum_{i=1}^{5} C_{i}^{k} \beta_{i}+C_{k} \alpha+C_{k}^{\prime} \varepsilon \leqslant F_{k}\left(\varepsilon_{1}, \varepsilon_{2}\right) \quad\left(k=1, \ldots, k_{M}\right) .
$$

Те из них, где $F_{k}>0$, не представляют опасности, так как их всегда можно удовлетворить при достаточно малых $\beta_{i}, \alpha, \varepsilon$. Остается убедиться, что непротиворечивы и условия, для которых $F_{k}=0$. Эти условия содержатся в (5.27), (5.33), (5.55), (5.64), и все они имеют вид

$$
\beta_{2} \geqslant C(p) \alpha+\sum_{i \neq 2} C_{i} \beta_{i}
$$


и также могут быть удовлетворены при достаточно малых $\beta_{i}, \alpha$. Таким образом, п. 1 леммы 5.1 доказан.

Доказательство п. 2 полностью аналогично. Нужно только учесть, что в (5.34)

$$
\begin{aligned}
A_{j}\left[w_{n m}^{ \pm}\left(1-\chi_{n m}^{ \pm}\right)\right] \leqslant & w_{n m}^{ \pm}\left(s_{c}^{1}\left(r_{0 j}\right), \vec{r}_{0 j}\right)+C \overline{\partial_{t} w_{n m}^{ \pm}} D_{0} \\
& +C \overline{\left(w_{n m}^{ \pm}\right)^{(1)}} \delta_{j}+C d_{0}^{-1} A(n) a^{j_{0}}
\end{aligned}
$$

В этой формуле $\partial_{t}=(\partial / \partial x, \partial / \partial y)$ и $\left|\partial_{t} w_{n m}^{ \pm}\left(s_{c}^{1}, \vec{r}_{0}\right)\right| \leqslant C\left(\bar{v}_{n}+\bar{v}_{n}^{(1)}\right) \frac{L|m|+|n|}{d_{0}^{2}}$ $\left(\vec{r}_{0}=(x, y)\right)$.

Аналоги условий $(5.62),(5.64)$ получаются при использовании значения $\delta_{j}(5.32)$ и имеют вид

$$
\begin{gathered}
\left(j_{0}-\frac{1}{2}\right) \beta_{5} \geqslant C(p) \alpha+\beta_{1}+\beta_{4}+\varepsilon, \quad \beta_{4}+\frac{1}{2} \beta_{5} \geqslant \varepsilon+C(\varkappa+1) \alpha \\
\beta_{2} \geqslant C_{1}(p) \beta_{1}+C_{2}(p, \varkappa) \alpha .
\end{gathered}
$$

Лемма 5.1 доказана.

5.3. Доказательство пп. 1, 2 теоремы 2.1. Если $\vec{r}_{0} \in D_{\beta_{1} \beta_{2}}^{1}$, из из (5.15) сразу следует нужная оценка ошибки дискретизации

$$
\left|\Delta \mu_{T}^{A}\left(\vec{r}_{0}\right)\right| \leqslant C G_{0}\left(A, \vec{r}_{0}\right) \sum_{s_{c}^{1}\left(\vec{r}_{0}\right)}\left|\Omega k\left(s_{c}^{1}\right)\right|^{-1 / 2} l\left(s_{c}^{1}\right)^{-1}+R,
$$

где величина $G_{0}$ определена в (2.24). Сравнение этой оценки с (2.27) показывает, что для доказательства п. 1 теоремы 2.1 достаточно получить удовлетворительную оценку ошибки регуляризации, т.е. показать, что $\Delta \mu_{R}^{A}\left(\vec{r}_{0}\right) \in\{O\}$. Вьше уже отмечалось (см. (3.2)), что величина $\widetilde{\mu}^{A}\left(\vec{r}_{0}\right)$ получается введением регуляризатора

$$
G(\xi)=R_{A, \Pi}(\lambda)=R^{A}(\lambda) \frac{1}{2}(\widehat{\Pi}(\lambda)+\widehat{\Pi}(-\lambda))=\frac{2 \pi}{\xi} S^{A}(\xi) S_{0}^{\Pi}(\xi)=2 \pi v_{0}(\xi)
$$

в формулу обрашения преобразования Фурье. Учитьвая (3.4), получим, что $\left\|G^{(N+1)}\right\|_{L^{1}} \leqslant C_{N} A_{0}$ при $\varkappa_{1}+\varkappa_{2}>2$. Для достаточно гладких алгоритмов можно взять $N=\min \left\{j_{0}, j_{1}\right\}$, и тогда оценка (П.29) удовлетворительна $\left(\Delta \mu_{R}^{A}\left(\vec{r}_{0}\right) \in\{O\}(5.7)\right)$ во всей рассматриваемой области $\vec{r}_{0}$, если

$$
\left(1-\beta_{1}\right) j_{0} \geqslant 2+\beta_{1}+2 \alpha, \quad\left(1-\beta_{1}\right) j_{1} \geqslant 2+\beta_{1}+2 \alpha
$$

Для достаточно гладких алгоритмов это условие выполняется при достаточно малых $\alpha, \beta_{i}$, и далее при доказательстве пп. 2, 3 теоремы 2.1 ошибки регуляризащии не рассматриваются.

Докажем п. 2 теоремы 2.1. Так как (см. (5.17), (5.63))

$$
B_{j}\left[w_{n m}^{ \pm}\right] \leqslant\left|w_{n m}^{ \pm}\left(s_{j}^{0}, \vec{r}_{0 j}\right)\right|+\left(\bar{v}_{n}+\overline{v_{n}^{(1)}}\right) \frac{L|m|+|n|}{d_{0}^{2}}\left(D_{0}+\frac{\bar{k} \delta_{j}}{d_{0}}\right)+\frac{C A(n) a^{j_{0}}}{d_{0}}
$$


то из $(5.16)$ с учетом выражения для $\delta_{j}(5.32)$ получим, что при $\vec{r}_{0} \in U\left(\beta_{2}, T\left(s_{j}^{0}\right)\right)$, $\left|\vec{r}_{0}-\vec{r}_{m}^{0}\right| \geqslant D^{\prime}$

$$
\begin{aligned}
\left|\Delta \mu^{A}\left(\vec{r}_{0}\right)\right| \leqslant & \sum_{s_{c}^{1}\left(\vec{r}_{0 j}\right)} \frac{G_{0}\left(A, \vec{r}_{0 j}\right)}{\left|\Omega k\left(s_{c}^{1}\left(\vec{r}_{0 j}\right)\right)\right|^{1 / 2} l\left(\vec{r}_{0 j}, s_{c}^{1}\left(\vec{r}_{0 j}\right)\right)}+\frac{G_{j}\left(A, \vec{r}_{0 j}\right)}{\left(\Omega K_{j}\right)^{1 /\left(p_{j}+2\right)} l\left(\vec{r}_{0 j}, s_{j}^{0}\right)} \\
& +\frac{\gamma A_{0} N_{1} a^{j_{0}-1 /\left(p_{j}+2\right)}}{L d_{0}\left(\Omega K_{j}\right)^{1 /\left(p_{j}+2\right)}}+\frac{\gamma A_{0}\left(D_{0}+\bar{k} d_{0}^{-1} \delta_{j}\right)}{L^{\varkappa} d_{0}^{2}\left(\Omega K_{j} a\right)^{1 /\left(p_{j}+2\right)}}+R \quad(\forall \gamma \geqslant 1) .
\end{aligned}
$$

Оценим третий и четвертый члены в правой части этой формулы. Третий член меньше $C \Omega^{-0.5-\varepsilon}$ при условии, что

$$
\beta_{5}\left(j_{0}-1 / 3\right) \geqslant \frac{1}{2}+C(p) \alpha+\beta_{4}+\varepsilon .
$$

Теперь заметим, что

$$
\begin{aligned}
& A_{0} D_{0} d_{0}^{-2} a^{-1 /\left(p_{j}+2\right)} L^{-\varkappa} \leqslant C \Omega^{-\beta^{\prime}}, \quad \bar{k} \delta_{j} d_{0}^{-3} a^{-1 /\left(p_{j}+2\right)} L^{-\varkappa} \leqslant C \Omega^{-\beta^{\prime}}, \\
& \beta^{\prime}=\min \left\{\beta_{2}-2 \beta_{1}+\frac{1}{3} \beta_{5}+C(\varkappa) \alpha, \frac{\beta_{4}}{p+1}+2 \beta_{1}+\frac{\beta_{5}}{(p+1)(p+2)}-\varkappa \alpha\right\},
\end{aligned}
$$

и, следовательно, при

$$
\beta^{\prime} \geqslant \varepsilon
$$

нужная оценка (2.29) следует из (5.67). Оценка (2.30) доказьвается точно так же, нужно только везде заменить $\vec{r}_{0 j}$ на $\vec{r}_{m}^{0}, D_{0}$ на $D^{\prime}, \delta_{j}$ на $\delta_{i}(i \subset(m))$ и ввести суммирование по $i \subset(m)(2.30)$. В частности, таким образом получаются аналоги оценок $(5.16),(5.67)$ в области $\left|\vec{r}_{0}-\vec{r}_{m}^{0}\right| \leqslant D^{\prime}=C \Omega^{-\beta_{3}}$.

Непротиворечивость всех условий справедливости оценок $(2.29),(2.30)$ и возможность их выполнения при достаточно малых $\alpha, \varepsilon_{0}$ в (2.26) для достаточно гладких алгоритмов (2.22) следует из явного вида этих условий и проверяется точно так же, как и при доказательстве п. 1. Таким образом, пункты 1, 2 теоремы 2.1 доказаны.

Заметим, что если в оценках $(2.27),(2.29)$ заменить $G_{0}$ и $G_{j}$ их оценками вида $C A_{0} L^{-\varkappa}(2.25)$, то полученные оценки будут транслящионно-инвариантными. Зависимость величин $G_{0}, G_{j}(2.24)$ от $\vec{r}_{0}$ позволяет качественно объяснить наблюдаемую геометрию артефактов (см. рис. 1). Рассмотрим, например, восстановление характеристической функции круга радиуса $B$ с центром в точке $\vec{a}$ при $|\vec{a}|>B$. Тогда центр сканирования $\vec{O} \notin \mathscr{D}$.

Рассмотрим случай $L \gtrsim 1, l\left(\vec{r}_{0}, s_{c}^{1}\right) \ll L$. Так как функции $S^{A}, S_{\Pi}$ достаточно быстро убывают, то основной вклад в величину $\Delta \mu_{T}^{A}\left(\vec{r}_{0}\right)$ дают члены с $m=0$, для которых (см. (4.11))

$$
\xi_{n 0}^{ \pm}\left(s_{c}^{1}\right)=-\frac{2 n\left\langle\vec{r}\left(s_{c}^{1}\right), \vec{l}\left(s_{c}^{1}\right)\right\rangle}{l^{2}\left(s_{c}^{1}\right)}, \quad \xi_{n 0}^{ \pm}\left(s_{c}^{1}\right)+2 n=-\frac{2 n\left\langle\vec{r}_{0}, \vec{l}\left(s_{c}^{1}\right)\right\rangle}{l^{2}\left(s_{c}^{1}\right)},
$$

а среди членов с $n \neq 0$ максимальный вклад дает член с $n=-1$. Условие $\xi_{n 0}^{ \pm}\left(s_{c}^{1}\right) \geqslant 0$ приводит к тому, что артефакты локализованыв области, где $\left(\left\langle\vec{r}\left(s_{c}^{1}\right), \vec{l}\left(s_{c}^{1}\right)\right\rangle\right)>0$. Это и объясняет эффект “притяжения” артефактов к центру сканирования, наблюдаемьй на томограммах (см. рис. 1). 
5.4. Доказательство п. 3 теоремы 2.1. Так же, как и при доказательстве леммы 5.1, будем последовательно оценивать интегралы $J_{n m}\left[\rho_{t} u_{n}\right]\left(\rho_{t}=\widetilde{\rho}, \rho_{i}, \rho_{0}\right)$ при $\vec{r}_{0} \in D_{\beta_{1} \beta_{2}}^{3}$.

5.4.1. Оценка интегралов $J_{n m}\left[\widetilde{\rho} u_{n}\right]$. Покажем, что $J_{n m}\left[\rho_{t} u_{n}\right] \in\{O\}$ при $\vec{r}_{0} \in D_{\beta_{1} \beta_{2}}^{3}\left(\rho_{t}=\widetilde{\rho}, \widetilde{\rho}_{i}\right)$. Начнем с оценки интеграла $J_{n m}\left[\widetilde{\rho} u_{n}\right]$. Как и в п. 5.2.2, будем исходить из представления (5.37) и использовать теорему П.2, но теперь величину $Q$ не фиксируем. При условии (5.39) получаем равенство

$$
J_{n m}\left[\widetilde{\rho} u_{n}\right]=\frac{2 \pi}{\Omega} \sum_{ \pm} \sum_{q=0}^{Q} \Omega^{-q} I_{1}^{ \pm}\left[f_{q}^{ \pm}\right]+\Delta_{Q T}\left[J_{n m}\left[\widetilde{\rho} u_{n}\right]\right]
$$

в котором интегралы $I_{1}^{ \pm}\left[f_{q}^{ \pm}\right]$задаются формулой $(5.21)$ и $f_{q}^{ \pm}(s)=l(s)^{-1} D_{q}(s)$, а величины $D_{q}^{ \pm}(s)$ определены в (П.6). При этом остаточный член $\Delta_{Q T}\left[J_{n m}\left[\widetilde{\rho} u_{n}\right]\right]$ допускает оценку $(\forall Q, T)$

$$
\left|\Delta_{Q T}\left[J_{n m}\left[\widetilde{\rho} u_{n}\right]\right]\right| \leqslant \frac{C_{Q, T}\left(\varepsilon_{1}, \varepsilon_{2}\right)}{\Omega d_{0}}\left(\frac{N_{1}}{a^{2} d_{0}^{2} \Omega}\right)^{Q+1}+\frac{C_{T}\left(\varepsilon_{1}, \varepsilon_{2}\right)}{\Omega^{T}} .
$$

Так как (см. (2.33)) $|[\vec{l}, \vec{\tau}]| \geqslant d_{T} \geqslant D_{0}$, то $\left|S^{(1)}(s)\right| \geqslant a d_{T}$, и интеграл $I_{1}^{ \pm}\left[f_{q}^{ \pm}\right]$можно оценивать, интегрируя по частям. Из (5.22), (5.63) следуют оценки

$$
\left|\left(D_{q}^{ \pm}\right)^{(p)}\right| \leqslant C_{p} A(n) \bar{k}\left(\frac{\bar{k} b N_{1}}{d_{0}^{2} a}\right)^{p}\left(\frac{(\bar{k})^{3} N_{1}}{d_{0}^{2} a^{2}}\right)^{q} .
$$

В (П.4) для интересующего нас случая можно взять $\lambda=N_{1} b^{-2}, \lambda_{1}=b d_{0}^{-1}, G(x) \geqslant$ $\widetilde{\Phi}_{1}^{2}=a^{2} d_{T}^{2}$, тогда получим, что $J_{n m}\left[\widetilde{\rho} u_{n}\right] \in\{O\}$ при условиях

$$
\begin{gathered}
\beta_{4}+\beta_{5}+\beta_{1}>\alpha, \quad C \alpha+2 \beta_{1}+\beta_{4}+2 \beta_{5}<1, \\
C \alpha+\beta_{4}+2 \beta_{5}+2 \beta_{2} \leqslant 1-\varepsilon^{\prime} \quad\left(\varepsilon^{\prime}>0\right) .
\end{gathered}
$$

Оценим интеграл $J_{n m}\left[\rho_{i} u_{n}\right]$ при $\xi_{i}^{n}+2 n=0$ и в этом случае будем писать $\rho_{i} \equiv \rho_{n}$. Так же, как в п. 5.2.4, используем следствие П.1 и в рассматриваемом случае $\lambda_{1}=$ $\lambda_{2}=1, \lambda=N_{1}, g_{1}=\bar{g}=\widetilde{g}=l, n_{0}=n_{1}=j_{0}, v_{|\alpha|}=A(n)$.

Из (П.65), (П.60) следует равенство

$$
\begin{aligned}
J_{n m}^{2}\left[\rho_{n} u_{n}\right]= & \sum_{c 1}\left(J_{n m}^{2}\left[\rho_{n} u_{n}\right]\right)_{c 1}^{0}+\sum_{c 2}\left(J_{n m}^{2}\left[\rho_{n} u_{n}\right]\right)_{c 2}^{Q} \\
& +\left(J_{n m}^{2}\left[\rho_{n} u_{n}\right]\right)_{0}^{P}+\Delta_{Q T}\left(J_{n m}^{2}\left[\rho_{n} u_{n}\right]\right) \\
& +\sum_{( \pm) \subset(1)} e^{i \Omega f\left(\varphi_{ \pm}\right)} \mathscr{K}_{ \pm} T_{\mathrm{ant}}^{0}
\end{aligned}
$$

$($ запись $( \pm) \subset(1)$ объяснена в $(5.47))$. Для величины $\Delta_{Q T}\left(J_{n m}^{2}\left[\rho_{n} u_{n}\right]\right)$ используем оценку (П.55) при $T=T(Q)$, граничньй член $\left(J_{n m}^{2}\left[\rho_{n} u_{n}\right]\right)_{0}^{P}$ оценивается с помошњю (П.50). Из (П.52), (П.64) при $N=N\left(\varepsilon_{1}, \varepsilon_{2}\right)$ получаем

$$
\left|\sum_{( \pm) \subset(1)} e^{i \Omega f\left(\varphi_{ \pm}\right)} \mathscr{K}_{ \pm} T_{\mathrm{ant}}^{0}\right| \leqslant C\left(\varepsilon_{1}, \varepsilon_{2}\right) \frac{A(n)}{\Omega d_{0}} t_{0}^{j_{0}}(\ln \Omega) \quad\left(t_{0}=C \Omega^{-\frac{1-\varepsilon_{1}}{2}} N_{1}^{\frac{5}{2}} d_{0}^{-3}\right) .
$$


Полагаем в (5.69) $Q=j_{0}$. Два первых члена в правой части (5.69) оцениваются сверху величинами

$$
C\left(\varepsilon_{1}, \varepsilon_{2}\right) A(n) t_{0}^{j_{0}}\left(\Omega d_{0}\right)^{-1}, \quad C\left(\varepsilon_{1} \varepsilon_{2}\right) A(n) a^{j_{0}}\left(\Omega d_{0}\right)^{-1} .
$$

Используя тривиальную оценку $\left|J_{n m}\left[\rho_{n} u_{n}\right]\right| \leqslant \mathscr{L} \max _{s}\left|J_{n m}^{2}\left[\rho_{n} u_{n}\right]\right|$, имеем

$$
\begin{aligned}
\left|J_{n m}\left[\rho_{n} u_{n}\right]\right| \leqslant & C\left(\varepsilon_{1}, \varepsilon_{2}\right) \mathscr{L} A(n) \\
& \times\left[\frac{a^{j_{0}}(\ln \Omega)}{\Omega d_{0}}+(\ln \Omega)\left(N_{1}^{5 / 2} \Omega^{-\frac{1-\varepsilon_{1}}{2}} d_{0}^{-2} a^{-2}\right)^{j_{0}}+\Omega^{-\frac{\varepsilon_{2}-\varepsilon_{1}}{3} j_{0}}\right]
\end{aligned}
$$

и, следовательно, $J_{n m}\left[\rho_{n} u_{n}\right] \in\{O\}$, если

$$
\begin{gathered}
\beta_{5} j_{0} \geqslant 2 \beta_{1}+2 \alpha+\beta_{4}, \quad j_{0}\left(\varepsilon_{2}-\varepsilon_{1}\right) \geqslant 3+2 \alpha+\beta_{4}, \\
j_{0}\left(\frac{1}{2}-\frac{\varepsilon_{1}}{2}-2 \beta_{1}-2 \beta_{5}+\frac{5}{2} \beta_{4}\right) \geqslant 3+2 \alpha+\beta_{4} .
\end{gathered}
$$

Осталось рассмотреть интеграл $J_{n m}\left[\rho_{i} u_{n}\right]$ при $\xi_{i}^{n}+2 n \neq 0$. В этом случае вместо (П.65) используем представление (П.68), из которого следует, что

$$
\begin{aligned}
J_{n m}^{2}\left[\rho_{i} u_{n}\right]= & \sum_{c 1}\left(J_{n m}^{2}\right)_{p, c 1}+\sum_{c 2}\left(J_{n m}^{2}\right)_{c 2}^{Q}+\sum_{( \pm) \subset(1)} e^{i \Omega f\left(\varphi_{ \pm}\right)} \mathscr{K}_{ \pm} T_{\mathrm{ant}}^{0} \\
& +\left(J_{n m}^{2}\left[\rho_{i} u_{n}\right]\right)_{0}^{P}+\Delta_{Q T}\left(J_{n m}^{2}\left[\rho_{i} u_{n}\right]\right)+\Delta^{p} J_{n m}^{2}
\end{aligned}
$$

и в этой формуле (см. (П.70))

$$
\left(J_{n m}^{2}\right)_{p, c 1}=i \pi e^{i \Omega f\left(\varphi_{ \pm}\right)} \sum_{q=0}^{p-1} T_{\mathrm{sym}}^{q} .
$$

Величина $\Delta^{p} J_{n m}^{2}$ в (5.71) оценивается с помошью (П.69) при $\lambda_{\varphi}=d_{0}^{-1}$. Остальные члены в правой части (5.71), кроме первого, оцениваются так же, как и вьше при $\rho_{i}=\rho_{n}$. Остается оценить интеграл

$$
\int\left(J_{n m}^{2}\right)_{p, c 1} d s=i \pi \sum_{q=0}^{p-1} \int e^{i \Omega S} T_{\mathrm{sym}}^{q} d s .
$$

Интегралы в правой части этого равенства оцениваются с помощью $p$-кратного интегрирования по частям (П.2), (П.4). Беря $p=P\left(\varepsilon_{1}, \varepsilon_{2}\right)$ и используя оценки

$$
\left|\left(\frac{d}{d s}\right)^{m} T_{\mathrm{sym}}^{q}\right| \leqslant C_{q}\left(\frac{\bar{k} b}{d_{0}^{2} t_{0}}\right)^{p} \bar{T}_{\mathrm{sym}}^{q}, \quad\left|T_{\mathrm{sym}}^{q}\right| \leqslant \bar{T}_{\mathrm{sym}}^{q}=\frac{C_{N_{q}} A(n) N_{1}^{q}}{\Omega^{q+1} d_{0}^{2 q} a^{4 q}},
$$

получим, что $J_{n m}\left[\rho_{i} u_{n}\right]=\{O\}$, если

$$
\begin{aligned}
& \beta_{4}+4 \beta_{5}+2 \beta_{2}+2 \beta_{1} \leqslant 1-\varepsilon^{\prime \prime}, \quad 2 \alpha+\beta_{5}+\beta_{1} \leqslant \frac{1}{2}+\frac{\varepsilon_{1}}{2}-\varepsilon^{\prime \prime}, \quad \varepsilon^{\prime \prime}>0, \\
& j_{1}\left(\frac{1}{2}-\frac{\varepsilon_{1}}{2}-2 \beta_{1}-2 \beta_{5}-\frac{5}{2} \beta_{4}\right) \geqslant 3+2 \alpha+\beta_{4}, \quad \frac{\varepsilon_{2}-\varepsilon_{1}}{2} j_{1} \geqslant 3+2 \alpha+2 \beta_{4} .
\end{aligned}
$$

Таким образом, при $\vec{r}_{0} \in D_{\beta_{1} \beta_{2}}^{3}$ основной вклад в величину $\Delta \mu_{T}^{A}\left(\vec{r}_{0}\right)$ дает интеграл $J_{n m}\left[\rho_{0} u_{n}\right]$. 
5.4.2. Оценка интеграла $J_{n m}\left[\rho_{0} u_{n}\right]$ и завершение доказательства теоремы 2.1. Случаи $n=0$ и $L|m| \geqslant 4|n|$ уже были рассмотрены вьше (см. пп. 5.2.3). Ниже предполагается, что выполнены условия (5.42), при которых справедливы оценки (5.43), (5.44), и рассматривается случай $L|m|<4|n|, n \neq 0$.

Для получения асимптотически точных оценок подход, используемьй при $\vec{r}_{0} \in$ $D_{\beta_{1} \beta_{2}}^{1} \cup D_{\beta_{1} \beta_{2}}^{2}$ и основанньй на представлении (5.45), не дает нужной точности, так как производная амплитуды, продолженной нулем в область $\xi<0$, имеет в точке $\xi=0$ разрыв первого рода и $j_{0 n}^{0}=j_{0 n}^{1}=1(2.20),(2.21)$. При $\vec{r}_{0} \in D_{\beta_{1} \beta_{2}}^{3}$ будем исходить из представления

$$
J_{n m}\left[\rho_{0} u_{n}\right]=\int_{0}^{2 \pi} d \varphi Y_{n m}^{2}\left[\rho_{0} u_{n}\right] e^{i \Omega f}
$$

в котором

$$
Y_{n m}^{2}\left[\rho_{0} u_{n}\right]=\int_{0}^{\infty} d \xi \int_{\partial \mathscr{D}} d s \rho_{0}(\xi) u_{n} e^{i \Omega \Psi}
$$

и функщии $f, \Psi$ определяются из соотношений

$$
f(\varphi)=-2 L m \varphi+2 n\left\langle\vec{r}_{0}, \vec{\eta}\right\rangle, \quad \Psi(\xi, s)=(\xi+2 n)\langle\vec{l}, \vec{\eta}\rangle .
$$

Фаза $\Psi$ как функция $\xi, s$ не имеет при $\xi \in \operatorname{supp} \rho_{0}, \vec{r}_{0} \in D_{\beta_{1} \beta_{2}}^{3}$, критических точек, и, учитывая определение (2.33) величины $d_{T}$, получаем

$$
G \equiv\left(\frac{\partial \Psi}{\partial \xi}\right)^{2}+\left(\frac{\partial \Psi}{\partial s}\right)^{2} \geqslant C d_{T}^{2}
$$

Интегрируя выражение для $Y_{n m}^{2}$ по частям, с помощью (П.2) получим равенство

$$
Y_{n m}^{2}\left[\rho_{0} u_{n}\right]=\sum_{j=1}^{N}\left(\frac{i}{\Omega}\right)^{j+1} Y_{n m}^{1}\left[w_{j}\right]+\Delta_{N} Y_{n m}^{2}
$$

в котором $Y_{n m}^{1}-$ одномерньй ОИ вида

$$
Y_{n m}^{1}\left[w_{j}\right]=\int_{\partial \mathscr{D}} w_{j} e^{i \Omega 2 n\langle\vec{l}, \vec{\eta}\rangle} d s, \quad w_{j}(\varphi, s)=\frac{\left.\langle\vec{l}, \vec{\eta}\rangle A_{\Psi}^{j}\left(u_{n}\right)\right|_{\xi=0}}{\langle\vec{l}, \vec{\eta}\rangle^{2}+4 n^{2}\langle\vec{\tau}, \vec{\eta}\rangle^{2}}
$$

Из (П.4), учитьвая, что для фазы $\Psi$ можно считать, что $\lambda=\bar{k} n b^{-2}, \lambda_{1}=b, G \geqslant C D_{0}^{2}$, при дополнительных условиях

$$
\beta_{6}>\alpha, \quad \beta_{2}>\beta_{6}+\beta_{4}+\alpha
$$

получим

$$
\left|\Delta_{N} Y_{n m}^{2}\right| \leqslant \Omega^{-N}\left|\int_{0}^{\infty} d \xi \int_{\partial \mathscr{D}} d s A_{\Psi}^{N}\left[\rho_{0} u_{n}\right]\right| \leqslant C_{N} k \mathscr{L} a_{0} b^{-1}\left(\frac{\bar{k} n}{D_{0}^{2} \Omega}\right)^{N}
$$


Подстановка разложения (5.74) в (5.73) дает

$$
J_{n m}\left[\rho_{0} u_{n}\right]=\sum_{j=1}^{N-1}\left(\frac{i}{\Omega}\right)^{N+1} I_{n m}\left[w_{j}\right]+\Delta_{N} J_{n m}\left[\rho_{0} u_{n}\right]
$$

В этой формуле $I_{n m}\left[w_{j}\right]-$ двумерньй ОИ вида

$$
\begin{gathered}
I_{n m}\left[w_{j}\right]=\int_{0}^{2 \pi} d \varphi \int_{\partial \mathscr{D}} d s e^{i \Omega \Phi(\varphi, s)} w_{j}, \\
\Phi(\varphi, s)=\Phi_{n m}(\varphi, 0, s)=-2 L m \varphi+2 n\langle\vec{r}, \vec{\eta}\rangle .
\end{gathered}
$$

Из (5.77) следует оценка остаточного члена $\Delta_{N} J_{n m}\left[\rho_{0} u_{n}\right]$ в (5.78):

$$
\left|\Delta_{N} J_{n m}\left[\rho_{0} u_{n}\right]\right| \leqslant C_{N} \mathscr{L} a_{0} b^{-1}\left(\frac{\bar{k} N_{1}}{D_{0}^{2} \Omega}\right)^{N}
$$

Таким образом, задача сводится к оценке ОИ $I_{n m}\left[w_{j}\right](5.7 .9)$. Для этого используется разбиение единицы по $s$

$$
1=\sum_{i} \rho_{i}(s)+\widetilde{\rho}(s)
$$

с центрами в точках уплощения $s_{i}^{0}$ и масштабами $\delta_{i}$ такими, что

$$
\begin{aligned}
\delta_{i} \geqslant \delta_{s}>0, & \delta_{i} \leqslant \frac{1}{2} \Delta_{i} \equiv C_{i} K_{i}\left(\bar{k} b^{p_{i}+1}\right)^{-1}, \\
\delta_{i} & \leqslant C K_{i}^{1 / p_{i}} .
\end{aligned}
$$

В этом случае имеются оценки

$$
\begin{array}{ll}
|k(s)| \geqslant k_{\delta}=\min \left\{C_{i} K_{i} \delta_{i}^{p_{i}}, \widetilde{k}\right\} & (s \in \operatorname{supp} \widetilde{\rho}), \\
|k(s)| \leqslant \frac{1}{2} & \left(s \in \operatorname{supp} \rho_{i}\right) .
\end{array}
$$

Запишем интеграл $I_{n m}\left[w_{j}\right]$ в виде

$$
I_{n m}\left[w_{j}\right]=\sum_{i} I_{n m}\left[w_{j} \rho_{i}(s)\right]+I_{n m}\left[w_{j} \widetilde{\rho}(s)\right]
$$

и будем оценивать интегралы $I_{n m}\left[w_{j} \rho_{i}(s)\right], I_{n m}\left[w_{j} \widetilde{\rho}(s)\right]$ по отдельности.

Начнем с интеграла $I_{n m}\left[\widetilde{\rho}(s) w_{j}\right]$ и используем представление

$$
\begin{gathered}
I_{n m}\left[\widetilde{\rho}(s) w_{j}\right]=\int_{0}^{2 \pi} e^{-i \Omega 2 L m \varphi} I_{n m}^{1} d \varphi \\
I_{n m}^{1}=\int_{\partial \mathscr{D}} e^{i \Omega_{1} \Phi(s)} \widetilde{\rho}(s) w_{j} d s, \quad \Omega_{1}=2 n \Omega, \quad \Phi(s)=\langle\vec{r}(s), \vec{\eta}\rangle .
\end{gathered}
$$


Покажем, что критические точки $s_{c}(\varphi)$ фазы $\Phi$ как функции одного переменного $s$ невырождены. Эти точки определяются из условий

$$
\vec{\nu}\left(s_{c}(\varphi)\right)=\varepsilon_{2} \vec{\eta}, \quad \varepsilon_{2}= \pm 1 \quad\left(\left\langle\vec{\tau}\left(s_{c}(\varphi)\right), \vec{\eta}\right\rangle=0\right)
$$

и они невырождены, так как $\Phi^{(2)}(s)=(k\langle\vec{\nu}, \vec{\eta}\rangle)(s)($ см. $(5.81))$ и

$$
\Phi^{(2)}\left(s_{c}(\varphi)\right)=k\left(s_{c}(\varphi)\right) \varepsilon_{2}, \quad\left|\Phi^{(2)}\left(s_{c}(\varphi)\right)\right| \geqslant k_{\delta} .
$$

Для исследования интеграла $I_{n m}^{1}$ используем теорему П.1. В рассматриваемом случае можно взять

$$
\delta_{c}=\delta_{0}, \quad \lambda^{c}=\lambda=\bar{k} b^{-2}, \quad \lambda_{1}^{c}=\lambda_{1}=b, \quad B_{0}=k_{\delta} .
$$

Так как $\Phi^{(1)}\left(s_{c x}\right)=1$, то величины $\delta_{c}$ достаточно малы при $k_{\delta} \delta_{0}<1$. Осталось еще определить величины $\lambda_{2}, \lambda_{2}^{c}, v_{j}(\Pi .5),(\Pi .8)$ такие, что

$$
\left|\partial_{s}^{m} w_{j}\right| \leqslant C_{j m} v_{j} \lambda_{2}^{-m}, \quad\left|\partial_{s}^{m} w_{j}\right| \leqslant C_{j m} v_{j}\left(\lambda_{2}^{c}\right)^{-m} \quad\left(\left|s-s_{c}(\varphi)\right|<2 \delta_{0}\right) .
$$

Предположим, что величины $\delta_{i}(5.80)$ удовлетворяют условиям

$$
\delta_{i}=\delta_{s}, \quad C \frac{D_{0}}{|n| b} \leqslant \delta_{s} \leqslant \frac{C}{b}
$$

Тогда можно взять

$$
v_{j}=A(n) \frac{\bar{k}}{b d_{T}^{2}}\left(\frac{\bar{k}|n|}{D_{0}^{2}}\right)^{j}, \quad \lambda_{2}=\left(\frac{d_{T}^{2}}{\bar{k} b n^{2}}\right)^{-1}, \quad \lambda_{2}^{c}=\left(\frac{d_{T}}{(\bar{k})^{1 / 2}|n| b}\right)^{-1} .
$$

Величина $\delta_{0}$ определяется в $\left(\right.$ П.9), т.е. $\delta_{0}=C \Omega^{-\frac{1-\varepsilon_{1}}{2}}(\bar{k})^{1 / 2}\left(k_{\delta}\right)^{-1}$. Теперь условия (П.10) имеют вид

$$
\frac{D_{0}^{2} k_{\delta}}{\bar{k} b|n|^{5 / 2}}>C \Omega^{-\frac{1-\varepsilon_{2}}{2}}, \quad \frac{(\bar{k})^{3} b^{2}}{\left(k_{\delta}\right)^{6}}<C \Omega^{1+\varepsilon_{2}}, \quad \frac{(\bar{k})^{5} b^{2}|n|^{5}}{D_{0}^{6}\left(k_{\delta}\right)^{3}}<C \Omega,
$$

и при их вьполнении для интеграла $I_{n m}^{1}$ имеет место представление (П.11). Используя это представление в (5.84) и переходя от переменной $\varphi$ к переменной $s(\varphi=\varphi(s) \Leftrightarrow$ $\langle\vec{\tau}, \vec{\eta}\rangle=0)$, получим

$$
\begin{aligned}
I_{n m}\left[\widetilde{\rho}(s) w_{j}\right]= & \sum_{\varepsilon_{2}}\left(\frac{2 \pi}{\Omega|n|}\right)^{1 / 2} e^{\frac{i \pi}{4} \varkappa_{c}} \sum_{t=0}^{Q} \frac{I_{2}\left[|k|^{1 / 2} D_{t}^{c}\left(\widetilde{\rho}(s), w_{j}\right)\right]}{(\Omega|n|)^{t}} \\
& +\Delta_{Q T}\left(I_{n m}\left[\widetilde{\rho}(s) w_{j}\right]\right) .
\end{aligned}
$$

В этой формуле $I_{2}[u]$ - одномерньй ОИ вида

$$
I_{2}[u]=\int_{\partial \mathscr{D}} e^{i \Omega_{1} S} u d s
$$


в котором

$$
\begin{gathered}
\Omega_{1}=-2 n \varepsilon_{2} \Omega, \quad S(s)=\gamma \varphi(s)-\langle\vec{r}(s), \vec{\eta}\rangle, \\
\gamma=\frac{L m}{n} \varepsilon_{2} .
\end{gathered}
$$

Величины $D_{t}^{c}\left[\widetilde{\rho}(s) w_{j}\right]$ задаются равенствами (П.6), и из (П.14) следует оценка

$$
\left|D_{t}^{c}\left[\widetilde{\rho}(s) w_{j}\right]\right| \leqslant C_{t} v_{6 t}\left(\frac{\bar{k} b^{2}|n|^{5}}{\left(d_{T}\right)^{6}\left(k_{\delta}\right)^{3}}\right)^{t}
$$

а для остаточного члена $\Delta_{Q T}\left(I_{n m}\left[\widetilde{\rho}(s) w_{j}\right]\right)$ имеется оценка $(\Pi .13)$ и $\forall T, Q$

$$
\Delta_{Q T}\left(I_{n m}\left[\widetilde{\rho}(s) w_{j}\right]\right) \leqslant \frac{C_{T}\left(\varepsilon_{1}, \varepsilon_{2}\right)}{\Omega^{T}}+\frac{C_{Q T}\left(\varepsilon_{1}, \varepsilon_{2}\right) \mathscr{N}_{2}}{|n|^{1 / 2} \Omega^{1 / 2} k_{\delta}}\left(\frac{(\bar{k})^{3} b^{2}|n|^{5}}{D_{0}^{6}\left(k_{\delta}\right)^{3} \Omega}\right)^{Q+1}
$$

где $\mathscr{N}_{2} \leqslant C \mathscr{L} \bar{k} b^{p+1} K_{0}^{-1}-$ число критических точек $s_{c}(\varphi)$.

Будем оценивать интеграл $I_{2}[u](5.89)$, в котором (см. (5.81))

$$
|k(s)| \geqslant k_{\delta} \quad(s \in \operatorname{supp} u), \quad|\vec{r}(s)| \leqslant \frac{1}{2}, \quad|\gamma|<4 .
$$

Так как функция $\varphi(s)$ определяется из условия $\langle\vec{\tau}, \vec{\eta}\rangle=0$, то $\frac{d \varphi}{d s}=-k(s)$. Величины $k(s), \varepsilon_{2}$ не меняют знака на связной компоненте $\operatorname{supp} \widetilde{\rho}$. Фаза $S$ не зависит от $\vec{r}_{0}$, и единственньм параметром является $\gamma$. Так как $S^{(1)}(s)=k(-\gamma+\langle\vec{r}, \vec{\eta}\rangle)$, то критические точки $s_{c}$ фазы $S(5.90)$ определяются из условий $\langle\vec{r}, \vec{\eta}\rangle=\gamma$. Эти точки сушествуют только при $|\gamma|<\frac{1}{2}$. Из (П.26) следует, что при $|\gamma| \geqslant 1$

$$
\left|I_{2}[u]\right| \leqslant \frac{c \bar{k} b^{2} \mathscr{L}}{\Omega|n|\left(k_{\delta}\right)^{2}}
$$

и ниже предполагается, что $|\gamma|<1$.

Так как $S^{(2)}\left(s_{c}\right)=k\left(s_{c}\right) f\left(s_{c}\right)$, где функция $f$ определена в $(2.11)$, то критическая точка $s_{c}$ вырождена тогда и только тогда, когда $s=s_{\alpha}$ где $s_{\alpha}$ - экстремальная точка функции $\langle\vec{r}, \vec{\tau}\rangle$ (см. (2.12)). Точки $s_{\alpha}$ зависят только от контура $\partial \mathscr{D}$ и отмеченной точки $\vec{O}$ - начала координат. Опишем алгоритм их нахождения. Пусть $\vec{r}_{E}(s) \in E, E$ - эволюта контура $\partial \mathscr{D}\left(\vec{r}_{E}(s)=\vec{r}(s)+(k(s))^{-1} \vec{\nu}(s)\right)$. Уравнение для точек $s_{\alpha}$ имеет вид $\left\langle\vec{r}_{E}\left(s_{\alpha}\right), \vec{\nu}\left(s_{\alpha}\right)\right\rangle=0$. Так как нормаль $\vec{\nu}(s)$ направлена по касательной к эволюте, то точка $s_{\alpha}$ определяется из условия: вектор $\vec{r}_{E}\left(s_{\alpha}\right)$ перпендикулярен к эволюте.

Единственной аналитической кривой, для которой точки $s_{\alpha}$ неизолированы, является окружность с центром в начале координат, и этот случай будет рассмотрен отдельно.

Условия вырождения $s_{c}=s_{\alpha}$ вьполняются только при $\gamma=\gamma_{\alpha}$, где величины $\gamma_{\alpha}$ определены в (2.13). Множество $\bigcup \gamma_{\alpha}$ является каустикой фазы $S(5.90)$, и точки $\gamma_{\alpha}$ предполагаются изолированньми. 
Оценка интеграла $I_{2}[u]$ производится с помощюю лемм П.1, П.2, а нужную информацию о локализации критических точек дает лемма П.3. Не останавливаясь на вычислениях, сформулируем результаты. Пусть $\gamma_{i} \subset \cup \gamma_{\alpha}-$ ближайшая к $\gamma$ точка каустики. Будем говорить, что $\alpha \subset i \quad(\alpha \neq i)$, если $\left|\gamma-\gamma_{\alpha}\right| \leqslant C f_{\alpha}^{q_{\alpha}+1}\left(\bar{k} b^{q_{\alpha}+1}\right)^{-q_{\alpha}-1}$. В области

$$
\left|\gamma-\gamma_{i}\right| \geqslant C \frac{\bar{k} b^{10 / 3} \mathscr{L}^{2 / 3}}{k_{\delta} \Omega_{1}^{1 / 4} F_{i}}, \quad F_{i}=\min _{\alpha \subset i}\left\{f_{\alpha}^{1 /\left(q_{\alpha}+1\right)}\right\}
$$

имеет место оценка

$$
\begin{gathered}
\left|I_{2}[u]\right| \leqslant \frac{C\left(\bar{u}+\bar{u}^{(\prime)} \mathscr{L}\right) \mathscr{N}_{3}}{\left|\Omega_{1} k_{\delta} \widetilde{f}_{i}(\gamma)\right|^{1 / 2}} \\
\widetilde{f}_{i}(\gamma)=\min _{\alpha \subset i}\left\{\tilde{f}, f_{\alpha}^{1 /\left(q_{\alpha}+1\right)}\left|\gamma-\gamma_{\alpha}\right|(\alpha \subset i)\right\} .
\end{gathered}
$$

Относительно используемых обозначений и определений величин $\tilde{f}, f_{\alpha}, f_{0}$ см. $\S 2$. В (5.93) $\mathscr{N}_{3}$ - число критических точек фазьљ $S$ (5.90). Оценка (5.93) получена с помошью леммы П.1, в которой $\Psi(s)=k_{\delta}(-\gamma+\langle\vec{r}, \vec{\eta}\rangle)$, и условия справедливости оценки (5.93) имеют вид

$$
\begin{gathered}
\frac{(\bar{k})^{3} b^{5} \mathscr{L}}{\left(k_{\delta}\right)^{3 / 2} \widetilde{f}^{3 / 2}} \leqslant C \Omega_{1}^{1 / 2}, \quad f_{\alpha}\left(\frac{f_{\alpha}}{\bar{k} b^{q_{\alpha}+1}}\right)^{q_{\alpha}+1} \geqslant C \frac{(\bar{k})^{2} b^{2} \mathscr{L} \tilde{f}^{4}}{\Omega_{1}\left(k_{\delta}\right)^{3}}, \\
\frac{\bar{k} b \mathscr{L}}{\Omega^{1 / 4}\left(k_{\delta}\right)^{3 / 2}} \leqslant C, \quad \tilde{f} \geqslant \frac{\bar{k} b^{10 / 3} \mathscr{L}^{2 / 3}}{k_{\delta} \Omega^{1 / 3}} .
\end{gathered}
$$

В области, где не выполняются условия (5.92), надо учитывать вырождение и использовать лемму П.2. В этой области

$$
\left|I_{2}[u]\right| \leqslant C \frac{\left(\bar{u}+\overline{u^{(1)}} \mathscr{L}\right) \mathscr{N}_{3}}{\left(\Omega_{1} k_{\delta} f_{i}\right)^{1 /\left(q_{j}+2\right)}}
$$

и эта оценка имеет место, если (см. (2.14))

$$
\begin{gathered}
\min _{\alpha \neq i}\left\{\left|\gamma_{\alpha}-\gamma_{i}\right|\right\} \geqslant \Delta^{0} \geqslant \frac{C_{i} \bar{k} b^{25 / 6} \mathscr{L}^{5 / 2}}{\left(k_{\delta}\right)^{3 / 2} F_{i}^{3 / 2} \Omega_{1}^{1 / 8}}, \\
\frac{(\bar{k})^{2 q_{i}+4} b^{2\left(q_{j}+1\right)^{2}+\frac{13}{3}}}{\left(k_{\delta}\right)^{2 q_{j}+5} f_{i}^{2 q_{i}+4} F_{i} \widetilde{f}^{2}} \leqslant C \Omega_{1}^{1 / 4}
\end{gathered}
$$

Так как $F_{i} \geqslant C_{i} f_{0}^{1 / 3}, \mathscr{N}_{3} \leqslant C_{q} \mathscr{L} \bar{k} b^{q+1} f_{0}^{-1}$, то из (5.93), (5.95) следует грубая, но равномерная по $\gamma$ оценка

$$
\left|I_{2}[u]\right| \leqslant \frac{C_{q}\left(\bar{u}+\overline{u^{(1)}} \mathscr{L}\right)}{\left(\Omega_{1} k_{\delta}\right)^{1 /(q+2)} f_{0}^{1 / 3}} \cdot \frac{\mathscr{L} \bar{k} b^{q+1}}{f_{0}},
$$

которая используется ниже. 
В интересующем нас случае $u(s)=k(s)^{1 / 2} D_{t}\left[\widetilde{\rho}(s) w_{j}\right]$. В неравенстве $\left|u^{(1)}(s)\right| \leqslant$ $\overline{u^{(1)}}$ можно взять $\overline{u^{(1)}}=C \lambda_{2} \bar{u}$, где величина $\lambda_{2}$ определена в (5.86). Из (5.88), (5.91), (5.97) при $Q=Q\left(\varepsilon^{\prime}, T\right)$ следует, что $\forall T$

$$
\left|I_{n m}\left[\widetilde{\rho}(s) w_{j}\right]\right| \leqslant \frac{C_{j T}\left(\varepsilon^{\prime}\right) A(n) \mathscr{L}^{2}(\bar{k})^{7 / 2} b^{q+1}|n|^{3 / 2}}{\Omega^{1 / 2+1 /(q+2)}\left(k_{\delta}\right)^{1 /(q+2)} d_{T}^{4} f_{0}^{4 / 3}}\left(\frac{\bar{k}|n|}{D_{0}^{2}}\right)^{j}+\frac{C_{j T}\left(\varepsilon^{\prime}\right) \mathscr{L} \bar{k} b^{q+1}}{f_{0} \Omega^{T}}
$$

и эта оценка верна при дополнительном условии

$$
\frac{(\bar{k})^{3} b^{2} N_{1}^{5}}{D_{0}^{6}\left(k_{\delta}\right)^{3} \Omega} \leqslant C \Omega^{-\varepsilon^{\prime}} \quad\left(\varepsilon^{\prime}>0\right) .
$$

Напомним, что рассматривается случай $L|m| \leqslant|n|$.

Рассмотрим специальный случай: $\partial \mathscr{D}$ - окружность радиуса $B$ с центром в начале координат. Возврашаясь в интеграле $I_{2}[u](5.89)$ к переменной $\varphi$, имеем

$$
\begin{gathered}
I_{2}\left[|k|^{1 / 2} D_{c}^{t}\left(\widetilde{\rho}(s) w_{j}\right)\right]=\int_{0}^{2 \pi} e^{i 2 L m \varphi} u_{t}(\varphi) d \varphi \\
u_{t}(\varphi)=\left(D_{c}^{t}|k|^{-1 / 2}\right)(s(\varphi)) .
\end{gathered}
$$

Отсюда сразу следует оценка

$$
I_{2}\left[|k|^{1 / 2} D_{c}^{t}\left(\widetilde{\rho}(s) w_{j}\right)\right] \leqslant \begin{cases}2 \pi \bar{u}_{t} & (m=0), \\ \frac{C\left(\bar{u}_{t}+\bar{u}_{t}^{(1)} \mathscr{L}\right)}{\Omega L|m|} & (m \neq 0),\end{cases}
$$

и вместо (5.98) получаем

$$
\left|I_{n m}\left[\widetilde{\rho}(s) w_{j}\right]\right| \leqslant \frac{C_{T j}\left(\varepsilon^{\prime}\right) A(n)}{\left(\Omega|n| k_{\delta}\right)^{1 / 2}}\left(\frac{\bar{k}}{b d_{T}^{2}}\right)^{j}+\frac{C_{T}\left(\varepsilon^{\prime}\right)}{\Omega^{T}} .
$$

Заметим, что эта оценка получена для любого значения $\delta_{s}$, удовлетворяющего условиям (5.80), (5.85).

Переходим к оценке интеграла $I_{n m}\left[\rho_{i}(s) w_{j}\right]$. По определению (5.83)

$$
\begin{aligned}
I_{n m}\left[\rho_{i} w_{j}\right] & =\int_{0}^{2 \pi} d \varphi \int_{\partial \mathscr{D}} d s e^{i \Omega \Phi(\varphi, s)} \rho_{i}(s) w_{j} \\
\Phi(\varphi, s) & =-2 L m \varphi+2 n\langle\vec{r}(s), \vec{\eta}\rangle .
\end{aligned}
$$

При условиях (5.80) критические точки $\left(\varphi_{c}, s_{c}\right)$ фазы $\Phi$ невырождены. Действительно, эти точки находятся из условий $\vec{\eta}\left(\varphi_{c}\right)=\varepsilon_{2} \vec{\nu}\left(s_{c}\right),\left\langle\vec{r}\left(s_{c}\right), \vec{\tau}\left(s_{c}\right)\right\rangle=\gamma$ и, следовательно, $\left|\operatorname{det} \Phi_{c}^{\prime \prime}\right|=4 n^{2}\left|1+k\left(s_{c}\right)\left\langle\vec{r}\left(s_{c}\right), \vec{\nu}\left(s_{c}\right)\right\rangle\right| \geqslant 2 n^{2}$, так как $\left|k\left(s_{c}\right)\right| \leqslant 0.5$ (5.82). Для оценки интеграла $I_{n m}\left[\rho_{i}(s) w_{j}\right]$ можно использовать теорему П.1, в которой $\delta_{c}=\delta_{0}$, $\lambda=|n| b^{-2}, \lambda_{1}=b, B_{c}=B_{0}=C|n|$, а условие достаточной малости $\delta_{c}$ имеет вид 
$\delta_{0} \leqslant C b^{-1}$. Это следует из того, что в области, где $\left|s-s_{c}\right|>\delta_{0}$ или $\left|\varphi-\varphi_{c}\right|>\delta_{0}$, справедлива оценка

$$
\left(\frac{\partial \Phi}{\partial \varphi}\right)^{2}+\left(\frac{\partial \Phi}{\partial s}\right)^{2} \geqslant C|n|^{2} \delta_{0}^{2} .
$$

Фигурируюшие в формулировке теоремы П.4 величины $\lambda_{2}, \lambda_{2}^{c}, v_{j}$ определяются равенствами (5.86), в которых надо положить $\bar{k}=1$. Непосредственно проверяется, что условия теоремы П.1 вьполняются, если вьполнены условия (5.87). Для оценки критического вклада используем (П.14). Число $\mathscr{N}_{4}$ критических точек $\left(\varphi_{c}, s_{c}\right)$ удовлетворяет условию $\mathscr{N}_{4}<2 \mathscr{N}_{3}$, а число $\mathscr{N}_{3}$ было оценено вьше. При условии (5.99), выбирая нужным образом $Q$ в (П.11), получим $(\forall T)$

$$
\left|I_{n m}\left[\rho_{i}(s) w_{j}\right]\right| \leqslant C_{j T}\left(\varepsilon^{\prime}\right) \frac{\mathscr{L} \bar{k} b^{q+1}}{f_{0}}\left[\frac{1}{\Omega^{T}}+\frac{A(n)}{\Omega|n| d_{T}^{2} b}\left(\frac{|n|}{D_{0}^{2}}\right)^{j}\right] .
$$

Оценка

$$
\left|I_{n m}\left[w_{j}\right]\right| \leqslant \frac{C_{j T}\left(\varepsilon^{\prime}\right) \mathscr{L}^{2}(\bar{k})^{7 / 2} b^{q+1}|n|^{3 / 2}}{\Omega^{1 / 2+1 /(q+2)}\left(k_{\delta}\right)^{1 /(q+2)} d_{T}^{4} f_{0}^{4 / 3}}\left(\frac{\bar{k}|n|}{D_{0}^{2}}\right)^{j}+\frac{C_{j T}\left(\varepsilon^{\prime}\right) \mathscr{L} \bar{k} b^{q+1}}{f_{0} \Omega^{T}}
$$

получается простьм сложением оценок (5.98), (5.101) с учетом оценки $\mathscr{N} \leqslant$ $C \mathscr{L}\left(\min \Delta_{i}\right)^{-1}(5.23)$ для числа $\mathscr{N}$ точек уплощения.

Выбирая в (5.78) $N=N\left(T, \varepsilon^{\prime}\right)$ и используя (5.102) при $L|m| \leqslant|n|$, имеем

$$
\left|I_{n m}\left[\rho_{0} u_{n}\right]\right| \leqslant \frac{C_{j T}\left(\varepsilon^{\prime}\right) \mathscr{L}^{2}(\bar{k})^{7 / 2} b^{q+1}|n|^{3 / 2}}{\Omega^{5 / 2+1 /(q+2)}\left(k_{\delta}\right)^{1 /(q+2)} d_{T}^{4} f_{0}^{4 / 3}}+\frac{C_{j T}\left(\varepsilon^{\prime}\right) \mathscr{L} \bar{k} b^{q+1}}{f_{0} \Omega^{T+2}} .
$$

Рассмотрим условия, при которых доказана эта оценка. Выбирая в соответствии с (5.80) $\delta_{s}=K_{0}\left(\bar{k} b^{p+1}\right)^{-1}$, получим, что условия (5.85) выполняются, если

$$
\beta_{2}>C(p) \alpha,
$$

и тогда (см. (2.33))

$$
k_{\delta}=\widetilde{K}_{p}=\min \left\{C \frac{K_{0}^{p+1}}{(\bar{k})^{p} b^{p^{2}}}, \widetilde{k}\right\} .
$$

Условия (5.99) выполняются, если

$$
5 \beta_{4}+6 \beta_{2}+C(p) \alpha \leqslant 1-\varepsilon^{\prime} .
$$

За исключением условий (5.76), (5.104) все условия справедливости (5.103), (5.87), (5.94), (5.96) также имеют вид (см. (5.10))

$$
C_{k}^{1} \beta_{4}+C_{k}^{2} \beta_{2}+C_{k}^{3}(q, p) \alpha<F_{k}^{\prime}\left(\varepsilon_{1}, \varepsilon_{2}\right), \quad F_{k}^{\prime}\left(\varepsilon_{1}, \varepsilon_{2}\right)>0 \text { при } \varepsilon_{i}<1 .
$$

Чтобы перейти к оценке величины $\Delta \mu_{T}^{A}\left(\vec{r}_{0}\right)$, к этим условиям надо добавить условия $(5.70),(5.72)$, которые выполняются для достаточно гладкого алгоритма, и условия $(5.68),(5.70),(5.39)$, при вьполнении которьх $J_{n m}\left[\rho_{t} u_{n}\right] \in\{O\} \quad\left(\rho_{t}=\widetilde{\rho}, \rho_{i}\right)$. Явньй вид всех перечисленных условий показьвает, что все их можно удовлетворить, зафиксировав достаточно малые положительные $\varepsilon^{\prime}, \varepsilon^{\prime \prime}, \varepsilon_{1}, \varepsilon_{2}$. 
Оценим вклад $\Delta \mu_{T F}^{A}\left(\rho_{0}\right)$ интегралов $I_{n m}\left[\rho_{0} u_{n}\right]$ в ошибку дискретизации:

$$
\begin{aligned}
\left|\Delta \mu_{T F}^{A}\left(\rho_{0}\right)\right| \equiv & \frac{\Omega}{2 \pi} \sum_{n, m}^{F}\left|J_{n m}\left[\rho_{0} u_{n}\right]\right| \leqslant \frac{\Omega}{2 \pi}\left[\sum_{|m| \neq 0}^{C N_{1} L^{-1}}\left|J_{0 m}\left[\rho_{0} u_{0}\right]\right|\right. \\
& \left.+\sum_{L|m| \geqslant C|n|}^{F} J_{n m}\left[\rho_{0} u_{n}\right]+\sum_{L|m|<C|n|}\left|J_{n m}\left[\rho_{0} u_{0}\right]\right|\right] .
\end{aligned}
$$

Используя оценки $(5.43),(5.44),(5.103)$ и выбирая нужным образом $T, \varepsilon^{\prime}$, при условиях $(5.42),(5.11)$ получим, что для обшего контура $\partial \mathscr{D}, \forall \gamma \geqslant 1$

$$
\left|\Delta \mu_{T F}^{A}\left(\rho_{0}\right)\right| \leqslant \gamma \frac{C\left(\varepsilon_{0}\right) A_{0} \mathscr{L}^{2}(\bar{k})^{2} b^{q+1}}{\Omega^{3 / 2+1 /(q+2)}\left(\widetilde{K}_{p}\right)^{1 /(q+2)} L f_{0}^{4 / 3}} .
$$

Эта оценка совпадает с первым членом оценки (2.31), и учитьвая, что при условии $(5.13) \Delta_{F} \mu^{A}\left(\vec{r}_{0}\right) \in\{O\}$, получим нужньй результат, так как $J_{n m}\left[\widetilde{\rho}_{t} u_{n}\right] \in$ $\{O\}\left(\rho_{t}=\widetilde{\rho}, \rho_{i}\right)$.

Доказательство оценки (2.35) полностью аналогично, надо только вместо (5.98) использовать (5.100). Так как пп. 1, 2 теоремы 2.1 были доказаны вьше, доказательство этой теоремы завершено.

С ЛЕДСТВИЕ 5.1. Пусть $\mu \in\left\{\mu^{\omega}, I\right\}, A \in\{A\}-$ достаточно гладкий алгоритм u $\sigma_{\infty}\left(\vec{r}_{0}\right)$ - асимптотический показатель сходимости (1.21). Тогда при $\vec{r}_{0} \notin \partial \mathscr{D}$

$$
\sigma_{\infty}\left(\vec{r}_{0}\right) \geqslant \begin{cases}\frac{1}{2} & \left(\vec{r}_{0} \in T(\partial \mathscr{D}), \quad \vec{r}_{0} \notin T_{0}\right) \\ \frac{1}{p+2} & \left(\vec{r}_{0} \in \cap T\left(s_{i}^{0}\right)\right), \quad p=\max p_{i} \\ \frac{3}{2}+\frac{1}{q+2} & \left(\vec{r}_{0} \notin T(\partial \mathscr{D})\right)\end{cases}
$$

ДоКАЗАТЕЛЬСТВо. Изложенньй в [19] метод разложения по гладкости позволяет заключить, что минимум показателя сходимости для заданного алгоритма $A \in\{A\}$ при $\mu \in\left\{\mu^{\omega}, I\right\}$ достигается на функциях вида $\mu=\chi \mathscr{D}$. Оценка (5.106) показателя сходимости прямо следует из теоремы 2.1 .

5.5. Формальный показатель сходимости и точность полученных оценок. Чтобы рассмотреть вопрос о точности полученных оценок, введем формальныи показатель сходимости $\sigma_{1}^{A}\left(\vec{r}_{0}\right)$. Сначала определим формальный показатель сходимости $\sigma_{1}^{T}\left(\vec{r}_{0}\right)$ для ошибки дискретизации. Пусть имеется асимптотически точное равенство

$$
J_{n m}\left[u_{n}\right]=O\left(\Omega^{-\gamma_{n m}\left(\vec{r}_{0}\right)}\right) \quad\left(\Omega \rightarrow \infty, m^{2}+n^{2} \geqslant 1, d\left(r_{0}, \partial \mathscr{D}\right)>0\right)
$$

в котором $m, n$ рассматриваются как непрерывные параметры. Тогда по определению

$$
\sigma_{1}^{T}\left(\vec{r}_{0}\right)=\left(\min _{m^{2}+n^{2} \geqslant 1}\left\{\gamma_{n m}\left(r_{0}\right)\right\}\right)-1
$$


Если, кроме того, для ошибки регуляризации $\Delta \mu_{R}^{A}\left(\vec{r}_{0}\right)$ имеется асимптотически точное равенство

$$
\Delta \mu_{R}^{A}\left(\vec{r}_{0}\right)=O\left(\Omega^{-\sigma_{1}^{R}\left(\vec{r}_{0}\right)}\right) \quad\left(d\left(\vec{r}_{0}, \partial \mathscr{D}\right)>0\right),
$$

то величина $\sigma_{1}^{A}\left(\vec{r}_{0}\right)$ определяется соотношением

$$
\sigma_{1}^{A}\left(\vec{r}_{0}\right)=\min \left\{\sigma_{1}^{T}\left(\vec{r}_{0}\right), \sigma_{1}^{R}\left(\vec{r}_{0}\right)\right\} .
$$

Предположим, что при суммировании по $n, m$ в выражении $(3.10)$ для $\Delta \mu_{T}^{A}\left(\vec{r}_{0}\right)$ не происходит сокращения главных членов асимптотики интегралов $J_{n m}\left[u_{n}\right]$, тогда $\sigma_{\infty}^{A}\left(\vec{r}_{0}\right)=\sigma_{1}^{A}\left(\vec{r}_{0}\right)$ и величина $\sigma_{1}^{A}\left(\vec{r}_{0}\right)$ может служить в качестве ориентира для оценки сверху величины $\sigma_{\infty}^{A}\left(\vec{r}_{0}\right)$.

Для гладкой амплитуды $u_{n}$ величину $\sigma_{1}^{T}\left(\vec{r}_{0}\right)$ можно вычислить с помощњю леммы 4.1 и формул (П.16), (П.17). При рассмотрении ОИ с негладкой амплитудой возникает вопрос об их равномерной оценке в области параметров, где критические точки близки к точкам нарушения гладкости амплитуды. Однако, если нас интересует оценка величины $\sigma_{1}^{T}\left(\vec{r}_{0}\right)$ сверху, то можно ограничиться рассмотрением следующих двух случаев: критические точки далеки от точек нарушения гладкости и критические точки совпадают с точками нарушения гладкости. Таким образом, можно использовать неравномерные (индивидуальные) оценки, т.е. оценки при фиксированных параметрах, учитьвая, что индивидуальный показатель сходимости не меньше равномерного. Теперь заметим, что теоремы П.1-П.5 с минимальными изменениями справедливы и в том случае, когда невырожденные критические точки попадают на гранищу области интегрирования. В этом случае надо только поставить коэффищиент $1 / 2$ у критического вклада такой точки и заменить $u$ на $\widetilde{\rho} u$ в граничных членах $J_{\partial M}^{P}$, $\left(J_{2}\right)_{0}^{P}$. Эти замечания вместе с леммой 4.1 и формулами (П.16), (П.17) позволяют оценить сверху формальньй показатель сходимости для негладких алгоритмов и контуров $\partial \mathscr{D}$. При этом надо действовать так же, как при доказательстве теоремы 2.1 , но учитьвать и граничные члены $J_{\partial M}^{P},\left(J_{2}\right)_{0}^{P}$ и т. д. Если предположить, что равномерньй показатель сходимости при приближении критических точек к точкам нарушения гладкости равен индивидуальному, то в приведенных ниже результатах можно взять знак равенства вместо оценки сверху, и тогда эти результаты дают точньй формальньй показатель сходимости и можно предположить, что это точньй асимптотический показатель сходимости для любого алгоритма $A \in\{A\}$ и кусочно-гладкого контура $\partial \mathscr{D}$.

Введем некоторые определения. Через $j$ обозначим нижнюю гранииу порядка нуля функиии $S^{A}$ в точках $\xi=2 k\left(\exists k\left(S^{A}\right)^{(m)}(2 k)=0, m \leqslant j-1,\left(S^{A}\right)^{(j)}(2 k) \neq 0\right)$. Напомним, что для сходяшихся алгоритмов $j \geqslant 1$. Рассмотрим функцию $\Psi_{ \pm}(s)=$ $2 L m \mp\left\langle\xi_{t}^{0} \vec{l}+2 n \vec{r}, \vec{\nu}\right\rangle$, где $\xi_{t}^{0}$ - точка разрьва первого рода функции $S^{A}$, если такая точка есть. Через $\widetilde{q}$ обозначим максимальньй по параметрам Lm, $n, \xi_{t}^{0}$ порядок нуля функиии $\Psi_{ \pm}$:

$$
\begin{gathered}
\Psi_{ \pm}\left(s_{c}\right), \ldots, \Psi_{ \pm}^{\left(n_{c}-1\right)}\left(s_{c}\right)=0, \quad \Psi_{ \pm}^{\left(n_{c}\right)}\left(s_{c}\right) \neq 0 \\
\widetilde{q}=\max _{ \pm, s_{c}, L m, n, \xi_{t}^{0}}\left\{n_{c}\right\} .
\end{gathered}
$$

Заметим, что для контура общего положения $\widetilde{q} \leqslant 5$. 
Теперь все готово, чтобы сформулировать результат вычислений.

Пусть $\mu=\chi_{\mathscr{D}}$ и контур $\partial \mathscr{D}$ состоит из конечного числа гладких дуг, каждая из которых имеет конечное число точек уплощения конечного порядка. $B$ этом случае справедливы следующие оченки сверху для величин $\sigma_{1}^{T}\left(\vec{r}_{0}\right), \sigma_{1}^{R}\left(\vec{r}_{0}\right)$ при $d\left(\vec{r}_{0}, \partial \mathscr{D}\right)>0$.

1. Если $\vec{r}_{0} \in T(\partial \mathscr{D})$, то

$$
\sigma_{1}^{R}\left(r_{0}\right) \leqslant \begin{cases}1 / 2, & \vec{r}_{0} \notin T_{0}, \\ 1 /(p+2), & \vec{r}_{0} \in \cap T\left(s_{i}^{0}\right), \quad p=\max \left\{p_{i}\right\}\end{cases}
$$

где $p_{i}$ - порядок точки уплощения (2.8).

2 . Если $\overrightarrow{r_{0}} \notin T(\partial \mathscr{D})$, контур $\partial \mathscr{D}$ не имеет угловых точек и функция $S^{A}$ непрерьвна, а число $q$ определено в (2.12), то

$$
\sigma_{1}^{+}\left(\vec{r}_{0}\right) \leqslant\left\{\begin{array}{ll}
1, & \left|\vec{r}_{0}\right|=0 \\
\frac{4}{3}, & \left|r_{0}\right| \neq 0
\end{array} \quad(j=1), \quad \sigma_{1}^{T}\left(\vec{r}_{0}\right) \leqslant \frac{3}{2}+\frac{1}{q+2} \quad(j \geqslant 2) .\right.
$$

Если функция $S^{A}$ непрерьвна, но у контура $\partial \mathscr{D}$ есть угловые точки, то

$$
\sigma_{1}^{T}\left(\vec{r}_{0}\right) \leqslant 1
$$

Если функция $S^{A}$ имеет разрывы первого рода и $\widetilde{q}$ определено в (5.107), то

$$
\sigma_{1}^{T}\left(\vec{r}_{0}\right) \leqslant \frac{1}{2}+\frac{1}{\widetilde{q}+1}
$$

3. Пусть $n_{0}, n$ - минимальные целые числа такие, что $G^{\left(n_{0}\right)}(0) \neq 0, G^{(n)}\left(\xi_{t}+0\right)-$ $G^{(n)}\left(\xi_{t}-0\right) \neq 0$, где $\xi_{t}-$ точка нарушения гладкости функции $G(5.66)$. Тогда

$$
\sigma_{1}^{R}\left(\vec{r}_{0}\right)=\min \left\{n_{0}, n+\beta\right\}, \quad \beta \geqslant 1 / 2,
$$

и для контура общего положения [25]

$$
\beta= \begin{cases}1 & \left(\vec{r}_{0} \notin E\right), \\ 5 / 6 & \left(\vec{r}_{0} \in E, \vec{r}_{0} \notin E_{0}\right), \\ 3 / 4 & \left(r_{0} \in E_{0}\right),\end{cases}
$$

где $E$ - эволюта $\partial \mathscr{D}$ и $E_{0}$ - объединение ее точек возврата.

Эти результаты показывают, что полученные в теореме 2.1 оченки точны в предположении, что не происходит сокращения главных членов асимптотик интегралов $J_{n m}\left[u_{n}\right]$ при суммировании по $n, m$. Заметим, что при восстановлении характеристической функции круга такого сокращения не происходит. 
5.6. Заключительные замечания. 1. Используя результаты [25] и $\S 4$, можно явно вьписать первые члены неравномерных (индивидуальных) асимптотик интегралов $J_{n m}\left[u_{n}\right]$ при $\Omega \rightarrow \infty \forall \vec{r}_{0} \notin \partial \mathscr{D}$. В частности, при $\vec{r}_{0} \in T(\partial \mathscr{D}), \vec{r}_{0} \notin T_{0}($ см. $\S 4$ и приложение)

$J_{n m}\left[u_{n}\right]=\left(\frac{2 \pi}{\Omega}\right)^{3 / 2} \sum_{ \pm} \sum_{s_{c}^{1}} \frac{e^{\frac{i \pi}{4} \varkappa_{c}+i \Omega S_{ \pm}\left(s_{c}^{1}\right)} u_{n}\left(\varphi_{c}^{1}, \xi_{c}^{1}, s_{c}^{1}\right)}{\left|k\left(s_{c}^{1}\right)\right|^{\frac{1}{2}}\left|\xi_{c}^{1}+2 n\right|^{1 / 2}}(\Omega \rightarrow \infty,(n, m) \neq(0,0))$.

Подстановка этой формулы в ряд (3.10) в случае, когда $\mathscr{D}-$ круг и $\vec{r}_{0} \notin \mathscr{D}$, показывает, что сокращения главных членов при суммировании по $n, m$ не происходит. Результат такой подстановки (формальньй ответ) удивительно хорошо описьвает картину артефактов, представленную на рис. 1.

2. От полученных при доказательстве теоремы 2.1 ограничений снизу на величины $j_{0}, j_{1}$, характеризующие гладкость алгоритма, можно избавиться, если учесть критические точки второго и третьего типов. Результаты, приведенные в приложении, позволяет это сделать. Соответствующие оценки для интеграла $I_{1}[u](5.21)$ не рассматривались только в целях экономии места. Проводя оценки более аккуратно, можно также существенно ослабить ограничения снизу на величину $d_{0}$, т.е. сузить область $U\left(\beta_{1}, \partial \mathscr{D}\right)$, где оценки не рассматриваются (см. рис. 2). Наибольшую сложность представляет вопрос об ослаблении требований на $\varkappa(2.18)$, т.е. на скорость убывания величины $S^{A}$ при $|\xi| \rightarrow \infty$. В сущности, этот вопрос упирается в необходимость ослабления условия (П.31) применимости теоремы П.3.

3. Все результаты в тексте были сформулированы для случая $\mu=\chi_{\mathscr{D}}$, где $\mathscr{D}-$ область с аналитической границей $\partial \mathscr{D}$. Если воспользоваться формулой

$$
\begin{aligned}
\iint_{\mathscr{D}} e^{i \lambda\langle\vec{r}, \vec{\eta}\rangle} f(\vec{r}, \varphi) d x d y & =\frac{i}{\lambda} \int_{\partial \mathscr{D}} e^{i \lambda\langle\vec{r}, \vec{\eta}\rangle} f(\vec{r}, \varphi)[\vec{\tau}, \vec{\eta}] d s \\
+ & \frac{i}{\lambda} \iint_{\mathscr{D}} e^{i \lambda\langle\vec{r}, \vec{\eta}\rangle}(\langle\vec{\nabla}, \vec{\eta}\rangle f)(\vec{r}, \varphi) d x d y \quad\left(\vec{\nabla}=\left(\frac{\partial}{\partial x}, \frac{\partial}{\partial y}\right)\right),
\end{aligned}
$$

то все эти результаты сразу переносятся на функции $\mu\left\{\mu^{\omega}, I\right\}$ вида (1.1) (cм. [19]). Требование аналитичности границы $\partial \mathscr{D}$ также можно существенно ослабить. Для применимости используемого метода при $\vec{r} \in D_{\beta_{1} \beta_{2}}^{2} \cup D_{\beta_{1} \beta_{2}}^{2}$ достаточно предположить, что $\partial \mathscr{D}$ состоит из конечного числа дуг ограниченной, но достаточно большой гладкости и каждая из этих дуг либо прямая, либо на ней имеется только конечное число точек уплощения конечного порядка. При $\vec{r}_{0} \in D_{\beta_{1} \beta_{2}}^{3}$ метод работает, если каждая из этих дуг - либо дуга окружности, либо функщия $f(2.11)$ на ней имеет конечное число нулей конечного порядка. Наличие угловых точек, где угол наклона касательной испытьвает скачок $\Delta_{\alpha}$ при $\vec{r}_{0} \in D_{\beta_{1} \beta_{2}}^{1} \cup D_{\beta_{1} \beta_{2}}^{2}$, вообше не изменяет результата, так как при выводе оценок в этой области мы сначала интегрировали по $\varphi, \xi$, а затем по $s$ и оценка интегралов по $s$ поводилась с помошью лемм П.1, П.2, которые, в сушности, не содержат ограничений на гладкость амплитуды. Для $\vec{r}_{0} \in D_{\beta_{1} \beta_{2}}^{3}$ при наличии угловых точек надо учесть граничные члены и критические точки второго типа. Для этого достаточно воспользоваться результатами из приложения, и для достаточно гладкого алгоритма получается оценка вида

$$
\left|\Delta \mu_{T}^{A}\left(\vec{r}_{0}\right)\right| \leqslant C \sum_{\alpha} \frac{\Delta_{\alpha}}{\Omega d_{T} L} \quad\left(\vec{r}_{0} \in D_{\beta_{1} \beta_{2}}^{3}\right),
$$


которая согласуется с (5.108).

\section{Приложение. Оценки осциллирующих интегралов}

В этом приложении без доказательств приведены все используемые в основном тексте сведения об оценках осциллирующих интегралов ОИ. Приложение содержит шесть пунктов. Результаты пш. 1-4 доказаны в работе [25]. Результаты, приведенные в пш. 5.6, по-видимому, являются новыми, и их доказательства будут опубликованы в другом месте.

1. Интегрирование по частям. Рассмотрим $n$-мерньй ОИ

$$
J[u]=\int_{M} e^{i \Omega \Phi(x)} u(x) d x \quad\left(x=\left(x_{1}, \ldots, x_{n}\right) \in \mathbb{R}^{n}\right)
$$

по параллелепипеду $M=\left\{x \in \mathbb{R}^{n} \mid a_{i} \leqslant x_{i} \leqslant b_{i}\right\}$. Квадрат нормы градиента $\nabla \Phi$ фазы $\Phi$ обозначим через $G\left(G=\sum_{i} \partial_{i} \Phi, \partial_{i}=\partial / \partial x_{i}\right)$. Через $A_{\Phi}$ обозначим оператор, действующий по формуле

$$
A_{\Phi}(f)=\sum_{i=1}^{n} \partial_{i}\left(f G^{-1} \partial_{i} \Phi\right)
$$

Если в носителе функции $u$ нет критических точек фазы $\Phi$ или в этих точках функция $u$ имеет ноль достаточно высокого порядка, то $\forall P \geqslant 0$ верна формула интегрирования по частям⿻

$$
J[u]=J_{\partial M}^{P}[u]+\left(\frac{i}{\Omega}\right)^{P} J\left[A_{\Phi}^{P} u\right]
$$

где граничньй член $J_{\partial м}^{P}[u]-$ сумма $(n-1)$-мерньх ОИ

$$
J_{\partial M}^{P}[u]=-\sum_{p=0}^{P-1}\left(\frac{i}{\Omega}\right)^{p+1} \sum_{k=1}^{n} \int_{M_{k}}\left[\left(A_{\Phi}^{p} u\right) G^{-1} \partial_{k} \Phi e^{i \Omega \Phi}\right]_{a_{k}}^{b_{k}} d \widehat{x}_{k}
$$

В этих формулах $\partial M=\bigcup M_{k}, M_{k}=\left\{x \in \mathbb{R}^{n} \mid x_{k}=a_{k}\right.$ или $\left.x_{k}=b_{k}\right\}$ и $d \widehat{x}_{k}=$ $d x_{1} \ldots d x_{k-1} d x_{k+1} \ldots d x_{n}$.

Если производные $\partial^{\alpha} \Phi\left(\partial^{\alpha}=\partial_{1}^{\alpha_{1}} \ldots \partial_{n}^{\alpha_{n}}, \alpha_{1}+\cdots+\alpha_{n}=|\alpha|\right)$ удовлетворяют условиям $\left|\partial^{\alpha} \Phi(x)\right| \leqslant C_{|\alpha|} \lambda \lambda_{1}^{|\alpha|}(2 \leqslant|\alpha| \leqslant N+1)$, то для степеней $A_{\Phi}^{N}$ оператора $A_{\Phi}$ справедлива оценка

$$
\left|\left(A_{\Phi}^{N} f\right)(x)\right| \leqslant C_{N n} \sum_{|\alpha|=0}^{N}\left|\partial^{\alpha} f(x)\right| \sum_{p=0}^{N-|\alpha|} G(x)^{-\frac{N+p}{2}} \lambda^{p} \lambda_{1}^{N-|\alpha|+p}
$$


2. Остаточный член в методе стационарной фазы. Рассмотрим $n$-мерньй ОИ (П.1) и будем предполагать, что все критические точки фазы $\Phi$ невырождены. Через $\langle x, y\rangle$ будем обозначать скалярное произведение в $\mathbb{R}^{n},|x|$ - норма элемента $x \in \mathbb{R}^{n}, d(x, U)$ - расстояние от $x$ до множества $U \subset \mathbb{R}^{n}$. Критическая точка $x_{c}$ $\left(\nabla \Phi\left(x_{c}\right)=0, \nabla=\left(\partial_{1} \ldots \partial_{n}\right)\right)$ называется невырожденной, если $\operatorname{det} \Phi_{c}^{\prime \prime} \neq 0$, где $\Phi_{c}^{\prime \prime}=\left(a_{i k}^{c}\right), a_{i k}^{c}=\partial_{i} \partial_{k} \Phi\left(x_{c}\right)$ - матрица вторых производных $\Phi$ в точке $x_{c}$. Через $B_{c}, B_{0}$ обозначим любые константы такие, что $0<B_{0} \leqslant B_{c} \leqslant\left\|\left(\Phi_{c}^{\prime \prime}\right)^{-1}\right\|^{-1}$, где $\|A\|$ - евклидова норма матрицы $A$. Фаза $\Phi$, как и амплитуда $u$, может зависеть от параметров $t=\left(t_{1}, \ldots, t_{m}\right)$. Предполагается, что в рассматриваемой области параметров $T(t \in T)$ фаза имеет конечное число невырожденных критических точек. Зависимости всех величин и оценок от $t$ ниже явно не указьваются.

Пусть фиксированы некоторые положительные числа $\delta_{c} \geqslant \delta_{0}>0$. Будем говорить, что эти числа $\delta_{c}$ достаточно малы, если минимум величины $G=(\nabla \Phi)^{2}$ в области $\left|x-x_{c}\right| \geqslant \delta_{c}$ достигается на одной из сфер $\left|x-x_{c}\right|=\delta_{c}$. Величины $\delta_{c}$ заведомо достаточно малы, если

$$
B_{c} \delta_{c} \leqslant G\left(x_{\mathrm{ex}}\right)^{1 / 2}, \quad B_{c} \delta_{c} \leqslant \min _{x \in \partial M} G(x)^{1 / 2}
$$

где $x_{\mathrm{ex}} \neq x_{c}$ - экстремальные точки функции $G\left(\nabla G\left(x_{\mathrm{ex}}\right)=0\right)$.

Будем предполагать, что $(u, \Phi) \in \Lambda_{\bar{\alpha}}\left(\lambda, \lambda_{1}, \lambda_{2}\right)$. Это означает, что для $u, \Phi$ имеют место оценки

$$
\begin{aligned}
& \left|\partial^{\alpha} \Phi(x)\right| \leqslant C_{|\alpha|} \lambda \lambda_{1}^{|\alpha|} \quad(2 \leqslant|\alpha| \leqslant \bar{\alpha}), \\
& \left|\partial^{\alpha} u(x)\right| \leqslant C_{|\alpha|} \lambda_{2}^{|\alpha|} v_{|\alpha|}(x), \quad v_{|\alpha|} \in L^{1} .
\end{aligned}
$$

Функции $h_{c}, \Psi_{\mu}^{c}$ и величины $D_{q}^{c}, b_{\mu, p}^{c}$ определим с помощью соотношений

$$
\left\{\begin{array}{l}
h_{c}(x)=\Phi(x)-\Phi\left(x_{c}\right)-\frac{1}{2}\left\langle\Phi_{c}^{\prime \prime}\left(x-x_{c}\right), x-x_{c}\right\rangle \\
\Psi_{\mu}^{c}(x)=u(x)\left(h_{c}(x)\right)^{\mu} \\
b_{\mu, p}^{c}=\frac{i^{p+\mu}}{\mu ! p ! 2^{p}}\left(\left\langle\left(\Phi_{c}^{\prime \prime}\right)^{-1} \nabla, \nabla\right\rangle^{p} \Psi_{\mu}^{c}\right)\left(x_{c}\right), \\
D_{q}^{c}=\sum_{\mu=0}^{2 q} b_{\mu, \mu+q}^{c} .
\end{array}\right.
$$

Кроме того, будем предполагать, что

$$
\begin{aligned}
& \left\|v_{j}\right\|_{L^{1}} \leqslant\|v\|_{m} \quad(j \leqslant m), \quad\left|v_{j}(x)\right| \leqslant \bar{v}_{m, c} \quad\left(j \leqslant m,\left|x-x_{c}\right| \leqslant 2 \delta_{c}\right), \\
& \left|v_{j}(x)\right| \leqslant \bar{v}_{j}^{c} \quad\left(\left|x-x_{c}\right| \leqslant 2 \delta_{c}\right)
\end{aligned}
$$

и в шаре $\left|x-x_{c}\right| \leqslant 2 \delta_{c}$

$$
\left|\partial^{\alpha} \Phi(x)\right| \leqslant C_{|\alpha|} \lambda^{c}\left(\lambda_{1}^{c}\right)^{|\alpha|}, \quad\left|\partial^{\alpha} u(x)\right| \leqslant C_{|\alpha|}\left(\lambda_{2}^{c}\right)^{|\alpha|} v_{|\alpha|}(x) .
$$


ТеОрема П.1. Пусть $(u, \Phi) \in \Lambda_{\bar{\alpha}}\left(\lambda, \lambda_{1}, \lambda_{2}\right)$, все критические точки $x_{c}$ невьрождень, их число $K \leqslant K_{M}$ и выполнень следующие условия:

1) величинь

$$
\delta_{c}=\delta_{0}=C_{n} \Omega^{-\frac{1-\varepsilon_{1}}{2}} \lambda^{1 / 2} \lambda_{1} B_{0}
$$

достаточно мальи и $d\left(x_{c}, \partial M\right) \geqslant 2 \delta_{0}, \delta_{0} \leqslant \lambda_{1}^{-1}<1$;

2) существуют не зависящие от $\Omega$ константы $\theta_{i}$ такие, что

$$
\|v\|_{\bar{\alpha}} \leqslant C \Omega^{\theta_{1}}, \quad \bar{v}_{\bar{\alpha}, c} \leqslant C \Omega^{\theta_{2}}, \quad \lambda \leqslant C \Omega^{\theta_{3}}, \quad K_{M} \leqslant C \Omega^{\theta_{4}} ;
$$

3) величины $\lambda, \lambda_{1}, \lambda_{2}, \Omega, B_{c}$ удовлетворяют условиям $\lambda_{1} \geqslant 1, \lambda \Omega \geqslant 1, \Omega \geqslant 1$ $u$

$$
\lambda^{5} \lambda_{1}^{12} B_{0}^{-6} \leqslant C \Omega^{1-\varepsilon_{2}}, \quad \lambda_{c}^{2}\left(\bar{\lambda}_{1}^{c}\right)^{2} B_{c}^{-3} \leqslant C \Omega,
$$

в которых $\varepsilon_{2} \geqslant \varepsilon_{1}>0, \bar{\lambda}_{1}^{c}=\max \left\{\lambda_{1}^{c}, \lambda_{2}^{c}\right\}$;

4) иелье числа $P, N, k, r$ определяются из соотношений

$$
\begin{aligned}
P & =\left[\left(T+\theta_{1}\right) \varepsilon_{1}^{-1}\right]+1, & N & =3 q, \quad r=2 q, \\
q & =\left[\left(T+\theta_{2}+\theta_{4}\right) \varepsilon_{2}^{-1}\right]+1, & k & =\left[\left(2 \theta_{3}+2+\varepsilon_{2}\right)\left(T+\theta_{2}+\theta_{4}\right)\left(\varepsilon_{2} \varepsilon_{1}\right)^{-1}\right]+1,
\end{aligned}
$$

где $T$ - любое фиксированное число и $[a]-$ челая часть числа а.

Тогда для любых таких $\varepsilon_{i}, \theta_{i}, T$ и любого $Q$ такого, что

$Q \leqslant[(1 / 3)(k-1)], \quad Q \leqslant[(1 / 2)(r-1)] \quad$ npu $\quad \bar{\alpha} \geqslant \max \{P+1,3 q+1,2 k+n+1\}$,

ОИ $Ј[u]$ (П.1) можнно представить в виде

$$
J[u]=\sum_{x_{c} \in M} J_{c}^{Q}+\Delta J_{Q T}+J_{\partial M}^{P}[u] .
$$

В этом представлении

$$
J_{c}^{Q}=\left(\frac{2 \pi}{\Omega}\right)^{\frac{n}{2}} \frac{e^{\frac{i \pi}{4} \varkappa_{c}+i \Omega \Phi\left(x_{c}\right)}}{\left|\operatorname{det} \Phi_{c}^{\prime \prime}\right|^{1 / 2}} \sum_{q=0}^{Q} \frac{D_{q}^{c}}{\Omega^{q}}
$$

где $\varkappa_{c}-$ индекс (разность между числом положительных и числом отрицательных собственных значений) матрицы $\Phi_{c}^{\prime \prime}$. Граничный член $J_{\partial м}^{P}[u]$ задается соотношением (П.3), и имеют место оченки

$$
\left|\Delta J_{Q T}\right| \leqslant C_{n}\left(Q, T, \varepsilon_{i}, \theta_{i}\right)\left[\Omega^{-T}+\sum_{x_{c}} \frac{\bar{v}_{2 k-2}^{c}}{\Omega^{n / 2}\left|\operatorname{det} \Phi_{c}^{\prime \prime}\right|^{1 / 2}}\left(\frac{\left(\lambda^{c}\right)^{2}\left(\bar{\lambda}_{1}^{c}\right)^{6}}{B_{c}^{3} \Omega}\right)^{Q+1}\right],
$$

$$
\left|D_{q}^{c}\right| \leqslant C_{n q} \bar{v}_{6 q}^{c}\left(\frac{\left(\lambda^{c}\right)^{2}\left(\bar{\lambda}_{1}^{c}\right)^{6}}{B_{c}^{3}}\right)^{q}
$$


Величина

$$
J_{c}^{0}=\left(\frac{2 \pi}{\Omega}\right)^{\frac{n}{2}} \frac{e^{i \Omega \Phi\left(x_{c}\right)+\frac{i \pi}{4} \varkappa_{c}}}{\left|\operatorname{det} \Phi_{c}^{\prime \prime}\right|^{1 / 2}} u\left(x_{c}\right)
$$

называется главным критическим вкладом точки $x_{c}$, и при $u\left(x_{c}\right) \neq 0$ величины $J_{c}^{0}$ определяют асимптотику ОИ $J[u]$ при $\Omega \rightarrow \infty$.

В работе [25] для одномерного случая получен явньй вид разложения $J[u]$ и для вырожденных критических точек (аналог теоремы П.1).

Пусть в критической точке $x_{c} \Phi^{(1)}\left(x_{c}\right)=0, \ldots, \Phi^{\left(\nu_{c}-1\right)}\left(x_{c}\right)=0, \Phi^{\left(\nu_{c}\right)}\left(x_{c}\right) \neq 0$ и $n=1$. Тогда легко посчитать порядок главного члена асимптотики критического вклада $J[u]$ точки $x_{c}$ при $\Omega \rightarrow \infty$. Предположим, что $n=1, u^{(p)}\left(x_{c}\right)=0, p \leqslant k-1$, $u^{(k)}\left(x_{c}\right) \neq 0$, тогда при нечетном $\nu_{c}$

$$
J_{c}^{Q}=C_{k, \nu_{c}}[u, \Phi] \Omega^{-\frac{k+1}{\nu_{c}}} \quad(\Omega \rightarrow \infty, n=1)
$$

Если $\nu_{c}$ - четное число и $u^{(p)}\left(x_{c}\right)=0(p \leqslant 2 j-2)$ или $u^{(2 j)}\left(x_{c}\right) \neq 0$, то

$$
J_{c}^{Q}=C_{j, \nu_{c}}[u, \Phi] \Omega^{-\frac{2 j+1}{\nu_{c}}} \quad(\Omega \rightarrow \infty, n=1) .
$$

3. Равномерные оценки одномерных ОИ. Рассмотрим одномерный ОИ

$$
J[u]=\int_{a}^{b} u e^{i \Omega \Phi(x)} d x \quad\left(x \in \mathbb{R}^{1}\right),
$$

в котором фаза и амплитуда могут зависеть от $m$-мерного параметра $t=\left(t_{1}, \ldots, t_{m}\right)$. Предполагается, что на интервале $[a, b]$ фаза имеет конечное число $\mathscr{N}$ критических точек $x_{c}$ конечной кратности:

$$
\Phi^{(1)}\left(x_{c}\right)=0, \quad \ldots, \quad \Phi^{\left(\nu_{c}-1\right)}\left(x_{c}\right)=0, \quad \Phi^{\left(\nu_{c}\right)}\left(x_{c}\right) \neq 0, \quad \nu_{c} \leqslant \nu
$$

и функция $\Phi$ по крайней мере $\nu+1$ раз непрерывно дифференцируема. Относительно амплитуды $u$ предполагается, что это ограниченная кусочно-дифференцируемая функция и $u^{(1)} \in L^{1}, \sum_{n}\left|\Delta_{n}\right|<\infty$, где $\Delta_{n}=u\left(x_{n}+0\right)-u\left(x_{n}-0\right), x_{n}$ - точка разрыва первого рода. В оценках используются следующие обозначения: $|u(x)| \leqslant \bar{u}$, $|u(x)| \leqslant \bar{u}_{c}\left(\left|x-x_{0}\right| \leqslant \delta_{c}, \delta_{c}>0\right), \sum_{n}^{c}\left|\Delta_{n}\right|=\sum_{n}\left|\Delta_{n}\right|\left(\left|x_{n}-x_{c}\right|<\delta_{c}\right)$.

В основном тексте для оценки одномерных ОИ используются две леммы, доказанные в [25] и являюшиеся следствием результатов, изложенных в работах [32], [33].

ЛЕмма П.1. Пусть при $t \in V$ выполнены условия 1-3.

1. Все критические точки $x_{c} \equiv x_{c}(t)$ невырождень $\left(\Phi^{(2)}\left(x_{c}\right) \neq 0\right)$ u $\Phi^{(2)} \in L^{1}$.

2. $\delta_{c} \geqslant \delta_{0}>0$ - любые положительнье числа, удовлетворяюшие условиям

$$
\delta_{c} \leqslant \Delta_{c} \leqslant \frac{1}{4}\left|\Phi^{(2)}\left(x_{c}\right)\right|\left(\bar{\Phi}_{c}^{(3)}(x)\right)^{-1} \quad\left(\left|\Phi^{(3)}(x)\right| \leqslant \bar{\Phi}_{c}^{(3)} \quad\left(\left|x-x_{c}\right| \leqslant \delta_{c}\right)\right) .
$$


3. При $x \in \operatorname{supp} u\left|\Phi^{(1)}(x)\right| \geqslant|\Psi(x)|$. Функиия $\Psi$ имеет по крайней мере две непрерьвнье производнье $и$

$$
\Psi_{\Delta}(t)=\min _{x_{\mathrm{ex}}}\left\{\left|\Psi\left(x_{\mathrm{ex}}\right)\right|,|\Psi(a)|,|\Psi(b)|\right\}
$$

где $x_{\mathrm{ex}}$ - экстремальнье точки функиии $\Psi \quad\left(\Psi^{(1)}\left(x_{\mathrm{ex}}\right)=0\right)$ u $x_{\mathrm{ex}} \neq x_{c}$.

Тогда при $t \in V$

$$
|J[u]| \leqslant C_{1} \sum_{x_{c}} \frac{A_{c}[u]}{\left|\Omega \Phi^{(2)}\left(x_{c}\right)\right|^{1 / 2}}+C_{2} \frac{A[u, \Phi]}{\Omega}
$$

и в этой оценке

$$
\begin{aligned}
A_{c}[u] & \leqslant\left|u\left(x_{c}\right)\right|+4 \delta_{c} \overline{u_{c}^{(1)}}+\sum_{n}{ }^{c}\left|\Delta_{n}\right|, \\
A[u, \Phi] & \leqslant\left(\bar{u}+\left\|\overline{u^{(1)}}\right\|_{L^{1}}+\sum_{n}\left|\Delta_{n}\right|\right)(\mathscr{N}+1) \Phi_{1}^{-1},
\end{aligned}
$$

а в качестве $\Phi_{1}$ можсно взять

$$
\Phi_{1}=\max _{x_{c}}\left\{\left|\Phi^{(2)}\left(x_{c}\right)\right| \delta_{c}, \frac{\left(\Psi^{(1)}\left(x_{c}\right) \Delta_{c}\right)^{2}}{\left\|\Phi^{(2)}\right\|_{L^{1}}}, \frac{\Psi_{\Delta}(t)^{2}}{\left\|\Phi^{(2)}\right\|_{L^{1}}}\right\} .
$$

Лемма П.1 применяется, когда значения параметра $t$ достаточно далеки от каустики. Напомним, что каустикой называется множество значений параметров, при которых фаза $\Phi$ имеет вырожденные критические точки.

Для оценки в окрестности каустики используется лемма П.2. Фиксируем некоторое значение параметров $t=t_{0}$ и введем обозначение $\Phi_{0}(x)=\Phi\left(x, t_{0}\right)$. Пусть $x_{0 c}-$ критические точки функции $\Phi_{0}$,

$$
\begin{gathered}
\left|\Phi_{0}^{(1)}(x)\right| \geqslant|\Psi(x)| \quad(x \in \operatorname{supp} u), \\
\Psi\left(x_{0 c}\right)=0, \quad, \ldots, \quad \Psi^{\left(\nu_{0 c}-2\right)}\left(x_{0 c}\right)=0, \quad \Psi^{\left(\nu_{0 c}-1\right)}\left(x_{0 c}\right) \neq 0
\end{gathered}
$$

и эти условия вьполняются при $\Psi=\Phi^{(1)}$.

Через $x_{\mathrm{ex}}$ снова обозначаются экстремальные точки функции $\Psi \quad\left(\Psi^{(1)}\left(x_{\mathrm{ex}}\right)=0\right.$, $\left.x_{\mathrm{ex}} \neq x_{0 c}\right)$ и величина $\Psi_{\Delta}$ определяется тем же равенством (П.20) при $t=t_{0}$.

ЛЕмма П.2. Пусть в окрестности $U_{0}$ точки $t_{0}$ фаза $\Phi$ имеет по крайней мере $\nu_{0 c}+1$ производную по $x$, эти производнье дифференцируем по $t_{k},\left(\partial_{k} \equiv \partial / \partial t_{k}\right) u$

$$
\begin{array}{ll}
\sum_{k=1}^{m}\left|\partial_{k} \Phi^{\left(\nu_{0 c}\right)}(x, t)\right| \leqslant D_{0 c}^{\nu} & \left.\left(\mid x-x_{0 c}\right) \leqslant \delta_{0 c}, t \in U_{0}\right), \\
\sum_{k=1}^{m}\left|\partial_{k} \Phi^{(1)}(x, t)\right| \leqslant D^{1} & \left(t \in U_{0}\right) .
\end{array}
$$


Пусть $\delta_{0 c}>0-$ - юбье числа такие, что

(П.24) $\delta_{0 c} \leqslant \Delta_{0 c} \leqslant \frac{1}{4} \frac{\left|\Phi_{0}^{\left(\nu_{0 c}\right)}\left(x_{0 c}\right)\right|}{\overline{\left(\Phi_{0}^{\left(\nu_{0 c}+1\right)}\right)_{c}}} \quad\left(\left|\Phi_{0}^{\left(\nu_{0 c}+1\right)}(x)\right| \leqslant\left(\overline{\Phi^{\left(\nu_{0 c}+1\right)}}\right)_{c},\left|x-x_{0 c}\right| \leqslant \delta_{0 c}\right)$,

и область $U_{0}$ такова, что при $t \in U_{0}$

$$
\begin{aligned}
D_{0 c}^{\nu}\left|t-t_{0}\right| & \leqslant \frac{1}{4}\left|\Phi_{0}^{\left(\nu_{0 c}\right)}\left(x_{0 c}\right)\right|, \\
D^{1}\left|t-t_{0}\right| & \leqslant \frac{1}{4} \widetilde{\Phi}_{01} \quad\left(|t|=\sqrt{t_{1}^{2}+\cdots+t_{m}^{2}}\right),
\end{aligned}
$$

где $\widetilde{\Phi}_{01}$ - любая величина, удовлетворяющая неравенству

$$
\widetilde{\Phi}_{01} \leqslant \min _{x_{0 c}}\left\{\frac{1}{2} \frac{\left|\Psi^{\left(\nu_{0 c}-1\right)}\left(x_{0 c}\right)\right| \delta_{0 c}^{\nu_{0 c}-1}}{\left(\nu_{0 c}-1\right) !}, \Psi_{\Delta}\right\}
$$

Тогда при $t \subset U_{0}$ имеет место оценка

$$
|J[u]| \leqslant \sum_{x_{0 c}} C\left(\nu_{0 c}\right) \frac{A_{0 c}[u]}{\left|\Omega \Phi_{0}^{\left(\nu_{0 c}\right)}\left(x_{0 c}\right)\right|^{1 / \nu_{0 c}}}+C \frac{A[u, \Phi]}{\Omega}
$$

в которой

$$
\begin{aligned}
& A_{0 c}[u] \leqslant\left|u\left(x_{0 c}\right)\right|+4 \delta_{0 c}\left(\overline{u^{(1)}}\right)_{0 c}+\sum_{n} 0 c\left|\Delta_{n}\right|, \\
& A[u, \Phi] \leqslant\left(\bar{u}+\left\|\overline{u^{(1)}}\right\|_{L^{1}}+\sum_{n}\left|\Delta_{n}\right|\right)(\mathscr{N}+1) \Phi_{01}^{-1},
\end{aligned}
$$

а в качестве $\Phi_{01}$ можнно взять любую величину такую, что

(П.28) $\Phi_{01} \leqslant \min _{x_{0 c}}\left\{\left|\Phi_{0}^{\left(\nu_{0 c}\right)}\left(x_{0 c}\right)\right| \delta_{0 c}^{\nu_{0 c}-1}, \frac{\left(\left|\Psi^{\left(\nu_{0 c}-1\right)}\left(x_{0 c}\right)\right|\left(\Delta_{0 c}\right)^{\nu_{0 c}-1}\right)^{2}}{\left\|\Phi_{0}^{(2)}\right\|_{L^{1}}}, \frac{\Psi_{\Delta}^{2}}{\left\|\Phi_{0}^{(2)}\right\|_{L^{1}}}\right\}$.

Необходимую для применения лемм П.1, П.2 информацию о локализации критических точек дает доказанная в [25]

ЛЕмма П.3. Пусть трансиендентное или алгебраическое уравнение $f(x)=0$ при $|x| \leqslant b$ приведено к виду

$$
x=a+R(x)
$$

$u|R(x)| \leqslant A|x|^{\sigma}$ при $\sigma>1$. Тогда

1. Если при некотором $\beta \leqslant 1 / 2$ выполняются условия

$$
a \leqslant \frac{1}{2}\left(\frac{\beta}{A}\right)^{1 /(\sigma-1)}, \quad|a| \leqslant(1-\beta) b
$$


то существует по крайней мере одно "близкое" к а решение $x_{0}$ уравнения $f(x)=$ 0, и для всех "близких" к а решений

$$
\frac{2}{3} a \leqslant\left|x_{0}\right| \leqslant 2|a|, \quad|x-a| \leqslant A(2|a|)^{\sigma} .
$$

Кроме того, возможно, имеются и “далекие" от а решения, для которых эти оченки не выполняются, и для них

$$
\left|x_{0}\right| \geqslant\left(\frac{1}{2 A}\right)^{\sigma-1} .
$$

2. Если $|a| \geqslant \frac{1}{2}\left(\frac{\beta}{A}\right)^{1 /(\sigma-1)}$, то для всех решений, если они существуют, верна оченка

$$
\left|x_{0}\right| \geqslant \frac{1}{3}\left(\frac{\beta}{A}\right)^{1 /(\sigma-1)} .
$$

4. Ошибка регуляризации. Полньй анализ ошибки регуляризации $\Delta \mu_{R}^{A}\left(\vec{r}_{0}\right)$ (1.26) проведен в работе [25]. В основном тексте используется простейшее следствие из результатов [25], которое мы сформулируем в виде леммы.

ЛЕмма П.4. Пусть функция $G(\xi)=\frac{1}{2} R^{A}(\lambda)(\widehat{\Pi}(\lambda)+\widehat{\Pi}(-\lambda)) \quad(\xi=\lambda / \Omega)$ непрерывна вместе с производными до $N$-го порядка включительно и $G^{(N+1)} \in L^{1}$. Eсли при этом $G^{(n)}(0)=0 \quad(n=1, \ldots, N)$ u $d\left(\vec{r}_{0}, \partial \mathscr{D}\right) \geqslant d_{0}$, mo

$$
\left|\Delta \mu_{R}^{A}\left(r_{0}\right)\right| \leqslant \frac{\mathscr{L}\left\|G^{(N+1)}\right\|_{L^{1}}}{\pi^{2} d_{0}\left(\Omega d_{0}\right)^{N}} .
$$

5. Специальный класс двумерных ОИ. Рассматриваемые специальные двумерные ОИ по полупространству - это интегралы вида

$$
\begin{gathered}
J_{2}[u]=\int_{0}^{\infty} d \xi \int_{0}^{2 \pi} d \varphi e^{i \Omega \Phi(x)} u(x), \\
\Phi(x)=f(\varphi)+\xi g(\varphi) \quad\left(x=\left(x_{1} x_{2}\right)=(\varphi, \xi)\right) .
\end{gathered}
$$

Будем говорить, что $J_{2} \in\left\{J_{2}\right\}$, если выполняются условия $1,2,3$.

1. Амплитуда $u$ - гладкая функция с компактньм носителем, периодическая по $\varphi$ с периодом $2 \pi$ :

$$
\begin{gathered}
u(\varphi, \xi)=0 \quad\left(\xi \geqslant \xi_{H}\right), \\
\left|\partial^{2} u(x)\right| \leqslant C_{|\alpha|} \lambda_{2}^{|\alpha|} v_{|\alpha|}(x), \quad \lambda_{1} \geqslant 1 \\
\left\|v_{|\alpha|}\right\| \leqslant C \Omega^{\theta_{1}}, \quad v_{|\alpha|}(x) \leqslant C \Omega^{\theta_{2}}
\end{gathered}
$$

2. Функции $f, g$ - гладкие периодические с периодом $2 \pi$ и

$$
\left|f^{(n)}(\varphi)\right| \leqslant C_{n} \bar{f} \lambda_{f}^{n} \quad(n \geqslant 2), \quad \begin{gathered}
\left|g^{(n)}(\varphi)\right| \leqslant C_{n} \bar{g} \lambda_{g}^{n} \quad(n \geqslant 1), \quad \lambda_{f} \geqslant 1, \quad \lambda_{g} \geqslant 1, \\
\bar{f}+\bar{g}+\xi_{H} \bar{g} \leqslant C \Omega^{\theta_{3}} .
\end{gathered}
$$


3. Критические точки $x_{c}=\left(\varphi_{c}, \xi_{c}\right)$ фазы $\Phi$ невырождены, т.е.

$$
\left|g^{(1)}\left(\varphi_{c}\right)\right| \geqslant g_{1}>0, \quad g_{1} \leqslant 1,
$$

где $\varphi_{c}$ определяется из условия $g\left(\varphi_{c}\right)=0$.

В этом случае имеет место первая оценка (П.5) и

$$
\lambda=\max \left\{f+\xi_{H} \bar{g}, \bar{g}\right\}, \quad \lambda_{1}=\max \left\{\lambda_{f}, \lambda_{g}\right\} .
$$

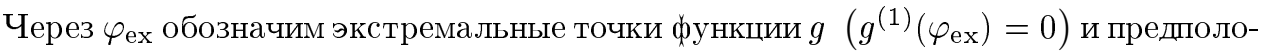
жим, что $\left|g\left(\varphi_{\text {ex }}\right)\right| \geqslant \widetilde{g}>0$.

Критические точки $x_{c}=\left(\varphi_{c}, \xi_{c}\right)$ определяются из условий

$$
g\left(\varphi_{c}\right)=0, \quad \xi_{c}=-f_{c}^{(1)} / g_{c}^{(1)} .
$$

Здесь и ниже для функций $f$ от $\varphi$ используется обозначение $f_{c}=f\left(\varphi_{c}\right)$.

Непосредственно проверяется, что $\operatorname{det} \Phi_{c}^{\prime \prime}=-\left(g_{c}^{(1)}\right)^{2}, \varkappa_{c}=0$,

$$
\Phi_{c}^{\prime \prime}=\left(\begin{array}{cc}
f_{c}^{(2)}+\xi_{c} g_{c}^{(2)} & g_{c}^{(1)} \\
g_{c}^{(1)} & 0
\end{array}\right), \quad\left(\Phi_{c}^{\prime \prime}\right)^{-1}=\left(\begin{array}{cc}
0 & \left(g_{c}^{(1)}\right)^{-1} \\
\left(g_{c}^{(1)}\right)^{-1} & -\left(g_{c}^{(1)}\right)^{-2}\left(f_{c}^{(2)}+\xi_{c} g_{c}^{(2)}\right)
\end{array}\right) .
$$

Так как фаза $\Phi$ может зависеть от дополнительных параметров, то при их изменении критические значения могут приближаться к нулю и пересекать границу области интегрирования. Введем величину $t^{0}=C \Omega^{-\frac{1-\varepsilon_{1}}{2}} g_{1}^{-2} \lambda^{3 / 2} \lambda_{1}^{3}$. Если $\left|\xi_{c}\right|>t^{0}\left(\forall x_{c}\right)$, то для анализа интеграла (П.30) можно применить теорему П.1. Так как в рассматриваемом случае $B_{c}=B_{0}=C g_{1}^{2} \lambda^{-1} \lambda_{2}^{-2}$, то первое из условий (П.10) приобретает вид

$$
\lambda \leqslant C \Omega^{\frac{1-\varepsilon_{2}}{11}} \lambda_{1}^{-24 / 11} g_{1}^{-12 / 11} .
$$

Это условие ограничивает возможный рост $\lambda$ при увеличении $\Omega$.

Если вьполняется дополнительное предположение $g_{c}^{(2)}=0$, то это условие можно ослабить и имеет место

ТЕОРемА П.2. Пусть $J_{2} \in\left\{J_{2}\right\},\left|\xi_{c}\right| \geqslant t^{0}, g_{c}^{(2)}=0$ и выполнены условия

$$
\begin{array}{ll}
\lambda \leqslant C \Omega^{\frac{1-\varepsilon_{2}}{5}} \lambda_{1}^{-\frac{12}{5}} g_{1}^{6 / 5}, & \lambda_{2}^{-1} \geqslant t^{0}, \quad \Omega \geqslant 2, \\
\tilde{g} \geqslant C \Omega^{-\frac{1-\varepsilon_{1}}{2}} \lambda_{1}^{1 / 2} \lambda & \left(t^{0}=C \Omega^{-\frac{1-\varepsilon_{1}}{2}} g_{1}^{-2} \lambda^{3 / 2} \lambda_{1}^{3}\right),
\end{array}
$$

u, кроме того, при $\bar{\lambda}_{1}=\max \left\{\lambda_{1}, \lambda_{2}\right\}$

$$
\begin{array}{lrl}
\lambda \lambda_{1}^{2}\left(\bar{\lambda}_{1}\right)^{2} g_{1}^{-2} \leqslant C \Omega, & g_{1} \lambda_{1}^{2} \leqslant C \lambda, \\
\lambda \geqslant C \Omega^{-1+\varepsilon_{3}}, & \bar{g} \lambda^{2} g_{1}^{-1} \leqslant C \Omega^{\theta_{3}} .
\end{array}
$$

Тогда для всех $\left(\theta_{i}, \varepsilon_{i}, T, Q\right)$ таких, что $0<\varepsilon_{i} \leqslant 1, \varepsilon_{2} \geqslant \varepsilon_{1}, T \geqslant 1, Q \geqslant 0 u$ выполняются условия (П.31), (П.32), при $P=P\left(\varepsilon_{i}, \theta_{i}\right) \geqslant 1$ имеет место равенство

$$
J_{2}[u]=\sum_{x_{c}}\left(J_{2}\right)_{c}^{Q}+\left(J_{2}\right)_{0}^{P}+\left(\Delta J_{2}\right)_{Q T}
$$


в котором суммирование ведется по критическим точкам $x_{c}=\left(\varphi_{c}, \xi_{c}\right), \xi_{c} \geqslant 0$,

$u$

$$
\left(J_{2}\right)_{c}^{Q}=\frac{2 \pi}{\Omega} \cdot \frac{e^{i \Omega \Phi\left(x_{c}\right)}}{\left|g_{c}^{(1)}\right|} \sum_{q=0}^{Q} \frac{D_{q}^{c}}{\Omega^{q}}
$$

$$
D_{q}^{c}=\sum_{\mu=0}^{q / 2} b_{\mu, \mu+q}^{c}, \quad\left|D_{q}^{c}\right| \leqslant C_{q} \bar{v}_{3 q}\left(\frac{\lambda \lambda_{1}^{2}\left(\bar{\lambda}_{1}\right)^{2}}{g_{1}^{2}}\right)^{q},
$$

где величины $b_{\mu, p}^{c}$ определень в (П.6). Граничный член $\left(J_{2}\right)_{0}^{P}$ имеет вид

$$
\left(J_{2}\right)_{0}^{P}=\left.\sum_{p=0}^{P-1}\left(\frac{i}{\Omega}\right)^{p+1} \int_{0}^{2 \pi} e^{i \Omega f} \frac{g}{\left(f^{(1)}\right)^{2}+g^{2}} A_{\Phi}^{p}(u)\right|_{\xi=0} d \varphi
$$

и для остаточного члена $\left(\Delta J_{2}\right)_{Q T}$ выполняется оценка

$$
\left(\Delta J_{2}\right)_{Q T} \leqslant C_{Q T}\left(\varepsilon_{i}, \theta_{i}\right)\left[\left(\frac{\lambda \lambda_{1}^{2}\left(\bar{\lambda}_{1}\right)^{2}}{g_{1}^{2} \Omega}\right)^{Q+1} \frac{B}{\Omega g_{1}}+\frac{1}{\Omega^{T}}\right] .
$$

При этом предполагается, что

$$
\left|v_{|\alpha|}\left(x_{c}\right)\right| \leqslant B
$$

Чтобы сформулировать результаты для случая, когда критические точки $x_{c}=$ $\left(\varphi_{c}, \xi_{c}\right)$ могут пересекать границу $\xi=0$ области интегрирования, введем еше ряд определений. Критические точки $x_{c}=\left(\varphi_{c}, \xi_{c}\right)$ будем делить на близкие $к$ граниче $x_{c 1}$, и далекие от нее $-x_{c 2}$, и для них соответственно

$$
\left|\xi_{c 1}\right| \leqslant t_{0}, \quad\left|\xi_{c 2}\right| \geqslant t_{0}, \quad t_{0}=C \Omega^{-\frac{1-\varepsilon_{1}}{2}} \lambda^{5 / 2}(\bar{g})^{3} \lambda_{1}^{7} g_{1}^{-7}>t^{0} .
$$

Пусть $\mathscr{N}_{1}$ - число близких, а $\mathscr{N}_{2}$ - число далеких от границы критических точек. Введем число $n_{0}$, характеризующее гладкость продолженной нулем в область $\xi<0$ амплитуды $u$, и будем предполагать, что

$$
\begin{gathered}
\partial_{\xi}^{n} u(\varphi, 0)=0 \quad\left(n \leqslant n_{0}-1\right), \quad \partial_{\xi}^{n_{0}} u(\varphi, 0) \neq 0, \\
\bar{n}_{0}= \begin{cases}n_{0} & \left(n_{0} \geqslant 2\right), \\
2 & \left(n_{0} \leqslant 2\right) .\end{cases}
\end{gathered}
$$

Будем считать, что кроме (П.37) выполняются оценки

$$
\left|v_{|\alpha|}\left(\varphi_{c 1}, 0\right)\right| \leqslant B_{0}, \quad\left|v_{|\alpha|}(\varphi, 0)\right| \leqslant B_{1} \quad\left(\left|\varphi-\varphi_{c 1}\right| \leqslant 2 t^{0}\right) .
$$

При формулировке и доказательстве теоремы П.3 используется несимметричное по переменным $\varphi, \xi$ разбиение единищы

$$
\widetilde{\rho}(\varphi, \xi)=1-\sum_{x_{c}} \omega\left(\frac{\varphi-\varphi_{c}}{\delta_{c}^{\varphi}}\right) \omega\left(\frac{\xi-\xi_{c}}{\delta_{c}^{\xi}}\right),
$$


в котором $\omega-$ гладкая функция $\left(\omega \in C_{0}^{\infty}\right)$ такая, что $\omega(t)=1(|t| \leqslant 1), \omega(t)=0$ $(|t| \geqslant 2)$, и при этом

$$
\delta_{c 1}^{\varphi}=\delta_{c 2}^{\varphi}=\delta_{c 2}^{\xi}=\delta_{0}=C t^{0}, \quad \delta_{c 1}^{\xi}=\delta_{1}=C \frac{g_{1}^{2}}{\lambda^{2} \lambda_{1}^{5}} .
$$

В окрестности $\left|\varphi-\varphi_{c}\right| \leqslant 2 \delta_{0}$ вместо $\varphi$ можно ввести переменную

$$
y=g(\varphi) \Leftrightarrow \varphi=\varphi(y)
$$

Функции от переменной $y-\rho_{c 1}, F, S$ определяются следуюшим образом:

$$
\rho_{c 1}(y)=\omega\left(\frac{\varphi(y)-\varphi_{c 1}}{\delta_{0}}\right), \quad F(y)=f(\varphi(y)), \quad S(y)=F(y)-F(0) .
$$

Через $\mathscr{K}\left[f e^{i \Omega S}\right]$ обозначается ОИ в смысле главного значения:

$$
\mathscr{K}\left[f e^{i \Omega S}\right]=\int_{0}^{\infty} y^{-1}\left[f(y) e^{i \Omega S(y)}-f(-y) e^{i \Omega S(-y)}\right] d y .
$$

ТЕОРема П.3. Пусть $J_{2} \in\left\{J_{2}\right\}$, выполняются условия (П.37)-(П.40) и, кроме moro,

$$
\begin{aligned}
& \lambda \leqslant C \Omega^{-\frac{1-\varepsilon_{2}}{11}} \lambda_{1}^{-\frac{24}{11}} g_{1}^{\frac{12}{11}}, \quad \lambda \leqslant C \Omega^{-\frac{1-\varepsilon_{1}}{9}} g_{1}^{\frac{16}{9}} \lambda_{1}^{-8 / 3}(\bar{g})^{-2 / 3}, \\
& \bar{g} \lambda_{2} g_{1}^{-1} \leqslant C \Omega^{\theta_{3}}, \quad \Omega \lambda \geqslant 1, \quad \Omega \geqslant 2, \quad \lambda \geqslant C \Omega^{-1+\varepsilon_{3}}, \\
& \tilde{g} \geqslant C \Omega^{-\frac{1-\varepsilon_{2}}{2}} \lambda_{1}^{1 / 2} \lambda, \quad \lambda_{2}^{-1} \geqslant C \Omega^{-\rho} \lambda_{1}^{3} g_{1}^{-7 / 12}\left((\bar{g})^{3}+\lambda^{-1 / 4}\right) .
\end{aligned}
$$

Тогда для любых $\varepsilon_{i}, \theta_{i}$ и любых $\sigma>0, \rho>0$ таких, что

$$
\begin{gathered}
\sigma<\frac{1}{4}\left(1+\varepsilon_{1}\right), \quad \sigma<\frac{1-\varepsilon_{1}}{2}-\frac{5}{22}\left(1-\varepsilon_{2}\right), \quad \varepsilon_{1} \leqslant \varepsilon_{2}, \quad 0<\varepsilon_{i} \leqslant 1, \\
\rho \leqslant \min \left\{\frac{1}{11}+\frac{5 \varepsilon_{2}}{66}, \frac{1}{4}+\frac{\varepsilon_{1}}{4}-\sigma, \frac{3}{22}-\frac{\varepsilon_{1}}{4}+\frac{5 \varepsilon_{2}}{44}, \frac{3}{11}-\frac{\varepsilon_{1}}{2}, \sigma+\frac{5 \varepsilon_{2}}{22}\right\},
\end{gathered}
$$

и любых $Q, T$ при $N=N\left(T, \varepsilon_{i}, \theta_{i}, \sigma\right), P=P\left(T, \varepsilon_{i}, \theta_{i}\right)$ справедливо равенство

$$
\begin{gathered}
J_{2}[u]=\sum_{x_{c 1}} \frac{i}{\Omega} e^{i \Omega f\left(\varphi_{c 1}\right)} \sum_{n=n_{0}}^{N-1} \frac{\partial_{\xi}^{n} u\left(\varphi_{c 1}, 0\right)}{n !} \xi_{c 1}^{n}\left[\mathscr{K}_{c 1}-i \pi\right] \\
+\sum_{x_{c 2}}\left(J_{2}\right)_{c 2}^{Q}+\left(J_{2}\right)_{0}^{P}+\Delta_{Q T}\left(J_{2}\right),
\end{gathered}
$$

в котором

$$
\mathscr{K}_{c 1} \equiv \mathscr{K}\left[\rho_{c 1} e^{i \Omega S}\right]
$$


и функции $\rho_{c 1}, S$ определены в (П.42). Граничный член

$$
\left(J_{2}\right)_{0}^{P}=\sum_{p=n_{0}}^{P-1}\left(\frac{i}{\Omega}\right)^{p+1} \int_{0}^{2 \pi} e^{i \Omega f(\varphi)} \frac{g}{\left(f^{(1)}\right)^{2}+g^{2}}\left(A_{\Phi}^{p}(\widetilde{\rho} u)\right)(\varphi, 0) d \varphi
$$

для $\Delta_{Q T}\left(J_{2}\right)$ выполняется оченка

$$
\left|\Delta_{Q T}\left(J_{2}\right)\right| \leqslant \frac{C_{Q T}\left(\varepsilon_{i}, \theta_{i}, \sigma\right) \mathscr{N}_{2} B}{\Omega g_{1}}\left(\frac{\lambda^{5} \lambda_{1}^{6}\left(\bar{\lambda}_{1}\right)^{6}}{g_{1}^{6} \Omega}\right)^{Q+1}+\frac{C_{T}\left(\varepsilon_{i}, \theta_{i}\right)}{\Omega^{T}}+\Delta^{n_{0}} J_{2}
$$

и по определению

$$
\begin{aligned}
\Delta^{n_{0}} J_{2}=C_{N} \mathscr{N}_{1}\left[\frac{B_{0}(\ln \Omega) E_{1}}{\Omega^{2+\frac{1-\varepsilon_{1}}{2}\left(\bar{n}_{0}-2\right)}+\frac{B_{1} E_{2}}{\Omega^{n_{0}+\frac{3}{2}-\frac{\varepsilon_{1}}{2}}}}\right. \\
\left.\quad+\frac{B_{0} E_{3} \Omega^{-\sigma \max \left\{0, n_{0}-1\right\}}}{\Omega^{\frac{3+\varepsilon_{1}}{2}}}+\frac{B_{0} E_{4}}{\Omega^{1+\frac{1-\varepsilon_{1}}{2}\left(n_{0}+1\right)}}\right]
\end{aligned}
$$

где в качестве констант $E_{i}=E_{i}\left(n_{0}, \lambda, \lambda_{1}, \lambda_{2}, \bar{g}, g_{1}\right)$ можно взять (П.49)

$$
\begin{aligned}
E_{1} & =\frac{\lambda(\bar{g})^{3} \lambda_{1}^{6} \lambda_{2}^{2 \bar{n}_{0}+2}}{g_{1}^{6}}\left(\frac{\lambda^{5 / 2}(\bar{g})^{3} \lambda_{1}^{7}}{g_{1}}\right)^{\bar{n}_{0}-2}, & E_{2} & =\frac{\bar{g} \lambda_{1}^{3} \lambda^{3 / 2} \lambda_{2}^{2 n_{0}+1}}{g_{1}^{2}}\left(\frac{\bar{g} \lambda_{1}^{2}}{g_{1}^{2}}\right)^{n_{0}+2} \\
E_{3} & =\frac{\bar{g} \lambda_{2}}{g_{1} \lambda^{3 / 2} \lambda_{1}}, & E_{4} & =\frac{1}{\lambda_{1}^{2} \lambda_{2}^{2}}\left(\frac{\bar{g}^{3} \lambda^{5 / 2} \lambda_{1}^{7} \lambda_{2}^{2}}{g_{1}^{6}}\right)^{n_{0}+1}
\end{aligned}
$$

Граничный член $\left(J_{2}\right)_{0}^{P}$ допускает следующую оценку:

$$
\left(J_{2}\right)_{0}^{P} \leqslant \frac{C_{P} B_{1}}{\Omega^{n_{0}\left(1+\varepsilon_{1}\right)}} \frac{g_{1}}{\Omega^{\frac{1+\varepsilon_{1}}{2}} \lambda^{3 / 2} \lambda_{1}^{3}} .
$$

Если ввести величину

$$
y_{0}=\frac{2}{3} g_{c 1}^{(1)} \delta_{0}
$$

(значение $\delta_{0}$ определено в $\left.(\Pi .41)\right)$, то

$$
\rho_{c 1}(y)=\left\{\begin{array}{ll}
1 & \left(|y| \leqslant y_{0}\right), \\
0 & \left(|y| \geqslant 6 y_{0}\right),
\end{array} \quad 0 \leqslant \rho(y) \leqslant 1\right.
$$

и величина $\mathscr{K}\left[\rho_{c 1} e^{i \Omega S}\right](\Pi .45)$ допускает следующую оценку:

$$
\mathscr{K}\left[\rho_{c 1} e^{i \Omega S}\right] \leqslant C \ln \Omega .
$$

Если $g_{c}^{(2)}=0$, то условия теоремы П.3 можно ослабить аналогично тому, как это было сделано при переходе от теоремы П.1 к теореме П.2, и тогда вместо теоремы П.3 получаем теорему П.4. 
ТеОРемА П.4. Пусть $J_{2} \in\left\{J_{2}\right\}, g_{c}^{(2)}=0$ и выполняются условия (П.37)-(П.40). Пусть, кроме того,

$$
\begin{aligned}
\lambda & \leqslant C \Omega^{\frac{1-\varepsilon_{2}}{5}} g_{1}^{6 / 5} \lambda_{1}^{-\frac{12}{5}}, \quad \Omega \lambda \geqslant 1, \quad \Omega \geqslant 2, \\
\widetilde{g} & \geqslant C \Omega^{\frac{1-\varepsilon_{1}}{2}} \lambda_{1}^{1 / 2} \lambda, \\
\lambda_{2}^{-1} & \geqslant C \Omega^{-\rho} \lambda_{1}^{4} g_{1}^{-3}\left(1+(\bar{g})^{4}+(\bar{g})^{1 / 2} \lambda^{-1 / 4}\right)
\end{aligned}
$$

и $\varepsilon_{i}, \sigma$-любые такие, что выполняются условия (П.53) $и$

$$
\begin{gathered}
\varepsilon_{2}>\varepsilon_{1}, \quad 0 \leqslant \varepsilon_{i}<1, \quad 0<\sigma<\frac{\varepsilon_{2}-\varepsilon_{1}}{2}, \quad \sigma<\frac{1+\varepsilon_{1}}{4}, \\
\rho=\min \left\{\frac{1}{5}+\frac{3 \varepsilon_{2}}{10}-\frac{\varepsilon_{1}}{2}, \frac{1+\varepsilon_{1}}{4}-\sigma, \frac{\varepsilon_{2}-\varepsilon_{1}}{4}, \frac{\varepsilon_{2}-\varepsilon_{1}}{2}-\sigma\right\} .
\end{gathered}
$$

Тогда $\forall Q, T, \theta_{i}$ при $N=N\left(T, \varepsilon_{i}, \theta_{i}, \sigma\right), P=P\left(T, \varepsilon_{i}, \theta_{i}\right)$ имеет место равенство (П.44), в котором

$$
\left|\Delta_{Q T}\left(J_{2}\right)\right| \leqslant \frac{C_{Q T}\left(\varepsilon_{i}, \theta_{i}, \sigma\right) \mathscr{N}_{2} B}{\Omega g_{1}}\left(\frac{\lambda \lambda_{1}^{2}\left(\bar{\lambda}_{1}\right)^{2}}{g_{1}^{2} \Omega}\right)^{Q+1}+\frac{C_{T}\left(\varepsilon_{i}, \theta_{i}\right)}{\Omega^{T}}+\Delta^{n_{0}} J_{2}
$$

(величина $\Delta^{n_{0}} J_{2}$ определена в (П.48)).

Наряду с интегралами $J_{2} \in\left\{J_{2}\right\}$ (П.30) рассмотрим интегралы $\widetilde{J} \in\left\{\widetilde{J}_{2}\right\}$ по всей прямой

$$
\widetilde{J}_{2}=\int_{-\infty}^{+\infty} d \xi \int_{0}^{2 \pi} u e^{i \Omega \Phi} d \varphi
$$

Предполагается, что выполнены те же условия на $u, \Phi$, что и при определении класca $\left\{J_{2}\right\}$, точка $\xi=0$ - единственная точка нарушения гладкости амплитуды $u$, и в этой точке производные $\partial_{\xi}^{n} u(\varphi, 0)$ непрерьвны при $n \leqslant n_{0}$ и

$$
\left(\Delta_{0} \partial_{\xi}^{n_{0}} u\right)(\varphi) \neq 0, \quad \text { где }\left(\Delta_{0} f\right)(\varphi) \equiv f\left(\varphi_{1}+0\right)-f\left(\varphi_{1}-0\right), \quad f=f(\varphi, \xi) .
$$

Функцию $G_{(\alpha)}$ от $y$ определим из формулы интегрирования сложной функции

$$
\begin{gathered}
\left(e^{i \Omega F(y)}\right)^{(j)}=\sum_{(\alpha)} C_{(\alpha)}(i \Omega)^{\alpha_{1}+\cdots+\alpha_{j}} e^{i \Omega F(y)} G_{(\alpha)}(y), \\
G_{\alpha}(y)=\left(F^{(1)}(y)\right)^{\alpha_{1}} \ldots\left(F^{(j)}(y)\right)^{\alpha_{j}} .
\end{gathered}
$$

В этой формуле $(\alpha)=\left(\alpha_{1}, \ldots, \alpha_{j}\right)$ и суммирование производится по всем наборам целых чисел $\alpha_{i} \geqslant 0$ таким, что $\alpha_{1}+2 \alpha_{2}+\cdots+j \alpha_{j}=j$.

Введем величину

$$
T_{j}(\Omega)=\sum_{(\alpha)} C_{(\alpha)}(i \Omega)^{\alpha_{1}+\cdots+\alpha_{j}} G_{(\alpha)}(0)
$$

и функщии

$$
h_{n}^{\mathrm{sym}}(y)=\frac{\partial_{\xi}^{n} u(\varphi,+0)+\partial_{\xi}^{n} u(\varphi,-0)}{\left|g_{1}(\varphi)\right|}, \quad h_{n}^{\mathrm{ant}}(y)=\frac{\left(\Delta_{0} \partial_{\xi}^{n} u\right)(\varphi)}{g_{1}(\varphi)}(\varphi=\varphi(y)) .
$$


ТеОрема П.5. Пусть $\widetilde{J}_{2} \in\{\widetilde{J}\}$ и выполнены условия (П.57). Тогда при тех же условиях, что и в теореме П.3 (теореме П.4) имеет место равенство

$$
\widetilde{J}_{2}[u]=\sum_{x_{c 1}} \widetilde{J}_{c 1, N}^{0}+\sum_{x_{c 2}}\left(\widetilde{J}_{2}\right)_{c 2}^{Q}+\left(\widetilde{J}_{2}\right)_{0}^{P}+\Delta_{Q T}\left(\widetilde{J}_{2}\right),
$$

в котором $\left(\widetilde{J}_{2}\right)_{c 2}^{Q}$ - критический вклад далеких от $\xi=0$ критических точек (П.12),

$$
\left(\widetilde{J}_{2}\right)_{0}^{P}=\sum_{n=n_{0}}^{P-1}\left(\frac{i}{\Omega}\right)^{p+1} \int_{0}^{\infty} e^{i \Omega f(\varphi)} \frac{g(\varphi)}{\left(f^{(1)}\right)^{2}+g^{2}}\left(\Delta_{0} A_{\Phi}^{p}(\widetilde{\rho} u)\right)(\varphi) d \varphi
$$

и для величины $\Delta_{Q T}\left(\widetilde{J}_{2}\right)$ справедлива оченка (П.47) $\left(\right.$ или (П.55) - при $\left.g_{c}^{(2)}=0\right), \boldsymbol{в}$ которой надо положить $E_{1}=0$ (П.48). Вклад $J_{c 1, N}^{0}$ близких $\kappa \xi=0$ критических точек $x_{c 1}$ определяется соотношениями

$$
\begin{gathered}
\widetilde{J}_{c 1, N}^{0}=i \pi e^{i \Omega f\left(\varphi_{c 1}\right)} T_{\mathrm{sym}}(\Omega)+e^{i \Omega f\left(\varphi_{c 1}\right)} \mathscr{K}_{c 1} T_{\text {ant }}(\Omega), \\
T_{\text {sym(ant) }}(\Omega)=\sum_{n=0}^{N-1}\left(\frac{i}{\Omega}\right)^{n+1} \sum_{j=0}^{n} \frac{\left(h_{n}^{\mathrm{sym}(\mathrm{ant})}\right)^{(n-j)}(0)}{j !(n-j !)} T_{j}(\Omega) .
\end{gathered}
$$

Опуская знаки sym, ant, разложим величину $T(\Omega) \equiv T_{\text {sym(ant) }}(\Omega)$ по обратным степеням $\Omega^{-1}$. При любом $m(1 \leqslant m \leqslant N-1)$

$$
T(\Omega)=\sum_{q=0}^{m} T^{q}+\Delta_{m} T, \quad T^{q}=\frac{1}{\Omega^{q+1}} \widetilde{T}^{q},
$$

где величины $\widetilde{T}^{q}$ не зависят от $\Omega$, и при условиях теоремы П.2

$$
\left|T^{q}\right| \leqslant \frac{C_{N q} B_{0} \lambda \lambda_{\varphi}}{(\lambda \Omega)^{q+1}}\left(\frac{\lambda \lambda_{\varphi}}{\lambda_{2}}\right)^{2 q}, \quad \lambda_{\varphi} \equiv \frac{\bar{g} \lambda_{1}^{2}}{g_{1}^{2}},
$$

а если выполнено условие

$$
\frac{\lambda \lambda_{\varphi}^{2} \lambda_{2}^{4}}{\Omega \lambda_{2}}<1
$$

то $\left|\Delta_{m} T\right| \leqslant\left(C_{N m} B_{0} \lambda^{m} \lambda_{\varphi}^{2 m} \lambda_{2}^{4 m}\right) / \Omega^{m+1}$.

Приведем формулы для $T^{0}, T^{1}$, в которых $h_{n} \equiv h^{\mathrm{sym}(\mathrm{ant})}$ :

$$
\begin{aligned}
& T^{0}=\frac{i}{\Omega} \sum_{n=0}^{N-1} \frac{h_{n}(0)}{n !} \xi_{c 1}^{n}, \\
& T^{1}=\frac{C_{1}}{\Omega^{2}} \sum_{n=1}^{N-1} \frac{(-1)^{n} h_{n}^{(1)}(0)}{(n-1) !} b_{1}^{n-1}+\frac{C_{2}}{\Omega^{2}} \sum_{n=2}^{N-1} \frac{(-1)^{n} h_{n}^{(1)}(0)}{(n-1) !} b_{1}^{n-2} b_{2}
\end{aligned}
$$

при $b_{k}=(k !)^{-1} F^{(k)}(0)$. 
СлЕДСТВИЕ П.1. Пусть выполнены условия теоремы (П.5) и условие $\partial_{\xi}^{n} u(\varphi, 0)=0 \quad\left(n \leqslant n_{1}\right)$. Тогда

$$
\begin{gathered}
\widetilde{J}_{2}[u]=\sum_{c 1}\left(\widetilde{J}_{2}\right)_{c 1}^{0}+\sum_{c 2}\left(\widetilde{J}_{2}\right)_{c 2}^{Q}+\left(J_{2}\right)_{0}^{P}+\Delta_{Q T}\left(J_{2}\right)+\Delta^{1} \widetilde{J}_{2} \\
+\frac{i}{\Omega} \sum_{c 1} e^{i \Omega f\left(\varphi_{c 1}\right)} \mathscr{K}_{c 1} \sum_{n=n_{0}}^{N-1} \frac{\left(\Delta_{0} \partial_{\xi}^{n} u\right)\left(\varphi_{c 1}\right)}{n !\left|g_{c 1}^{(1)}\right|} \xi_{c 1}^{n} .
\end{gathered}
$$

В этой формуле $\left(\widetilde{J}_{2}\right)_{c 1}^{0}$ - основной критический вклад точки $\left(\varphi_{c 1}, \xi_{c 1}\right)$, вычисляемый по формуле (П.12) при $Q=0$, и при этом по определению $u\left(\varphi_{c 1}, \xi_{c 1}\right)=$ $\frac{1}{2}\left(u\left(\varphi_{c 1}, \xi_{c 1}+0\right)+u\left(\varphi_{c 1}\right)\right)$. Для $\Delta_{Q T}\left(J_{2}\right)$ верны те же оценки, что и в теореме П.4, u

$$
\begin{aligned}
& \left|\Delta^{1} \widetilde{J}_{2}\right| \leqslant \frac{C_{N n_{1}} B_{0} \mathscr{N}_{1}}{\Omega^{2}} \cdot \frac{\lambda \lambda_{\varphi}^{3}}{\lambda_{2}^{2 \bar{n}_{1}+2}} t_{0}^{\bar{n}_{1}-2}, \\
& \bar{n}_{1}=\left\{\begin{array}{ll}
n_{1} & \left(n_{1} \geqslant 2\right), \\
2 & \left(n_{1} \leqslant 2\right),
\end{array} \lambda_{\varphi}=\frac{\bar{g} \lambda_{1}^{2}}{g_{1}^{2}} .\right.
\end{aligned}
$$

В случае $n_{1}=0$ этот результат может оказаться недостаточно точным и можно использовать следуюшее представление, имеющее место $\forall m(1 \leqslant m \leqslant N-1)$ :

$$
\begin{aligned}
\widetilde{J}_{2}[u]= & \sum_{c 1} \widetilde{J}_{2, m, c 1}+\sum_{c 2}\left(\widetilde{J}_{2}\right)_{c 2}^{Q}+\sum_{c 1} e^{i \Omega f\left(\varphi_{c 1}\right)} \mathscr{K}_{c 1} T_{\text {ant }}^{0} \\
& +\left(\widetilde{J}_{2}\right)_{0}^{P}+\Delta_{Q T}\left(J_{2}\right)+\Delta^{m} \widetilde{J}_{2} .
\end{aligned}
$$

В этой формуле

$$
\left|\Delta^{m} \widetilde{J}_{2}\right|=\left|\Delta_{m} T^{\mathrm{sym}}\right| \leqslant \frac{C_{N m} B_{0} \lambda^{m} \lambda_{\varphi}^{2 m} \lambda_{2}^{4 m}}{\Omega^{m+1}}
$$

а величина $\widetilde{J}_{2, m, c 1}$ определяется равенством

$$
\widetilde{J}_{2, m, c 1}=-i \pi e^{i \Omega f\left(\varphi_{c 1}\right)} \sum_{q=0}^{m-1} T_{\mathrm{sym}}^{q}
$$

6. Оценки главных значений ОИ. В этом пункте приведены используемые в основном тексте оценки интегралов $\mathscr{K} \equiv \mathscr{K}\left[\rho e^{i \Omega S}\right]$, понимаемых в смысле главного значения (П.43).

Предполагается, что выполнены условия 1,2 .

1. $\rho \in C_{0}^{\infty}$ и

$$
\rho(y)=\left\{\begin{array}{ll}
1 & \left(|y| \leqslant y_{0}\right), \\
0 & \left(|y| \geqslant C y_{0}\right),
\end{array} \quad\left|\rho^{(k)}(y)\right| \leqslant C_{k} y_{0}^{-k}, \quad y_{0} \leqslant 1, \quad y_{0}^{-1} \geqslant C \Omega^{\theta} .\right.
$$

2. Фаза $S$ - гладкая в интервале $|y| \leqslant C y_{0}$ функция и $S(0)=0$. 
Используются обозначения

$$
\begin{aligned}
b_{k} & =(k !)^{-1} S^{(k)}(0), & S_{0}(y) & =b_{1} y_{1}+b_{2} y_{2}, \\
\mathscr{K}_{0} & =\mathscr{K}\left[\rho e^{i \Omega S_{0}}\right], & A_{0} & =\mathscr{K}\left[e^{i \Omega S_{0}}\right] .
\end{aligned}
$$

Интеграл $A_{0}$ вьчисляется аналитически:

$$
\begin{aligned}
& A_{0}=i \pi \operatorname{sgn} b_{1} \operatorname{erf}\left[\sqrt{x} e^{\frac{i \pi}{4} \operatorname{sgn} b_{1}}\right] \quad\left(x=\frac{\Omega b_{1}}{4\left|b_{2}\right|}\right), \\
& \operatorname{erf}(z)=\frac{2}{\pi} \int_{0}^{z} e^{-t^{2}} d t \quad\left(\operatorname{sgn} b_{1}=0 \quad\left(b_{1}=0\right)\right),
\end{aligned}
$$

и его можно представить в виде

$$
A_{0}=i \pi \operatorname{sgn} b_{1}+\Delta A_{0}
$$

При этом, если $x>C \Omega^{\varepsilon^{\prime}}$, то $\forall T, \varepsilon^{\prime}>0$

$$
\begin{gathered}
\Delta A_{0}=\frac{1}{\sqrt{x}}\left[e^{i x \operatorname{sgn} b_{1}} \cdot\left\{\begin{array}{ll}
i & \left(b_{1}>0\right) \\
0 & \left(b_{1}<0\right)
\end{array}\right] \sum_{m=0}^{m_{T}\left(\varepsilon^{\prime}\right)} a_{m} x^{-m}+\Delta_{T}\left(\varepsilon^{\prime}, \theta\right),\right. \\
\Delta_{T}\left(\varepsilon^{\prime}, \theta\right) \leqslant C_{T}\left(\varepsilon^{\prime}, \theta\right) \Omega^{-T} .
\end{gathered}
$$

С другой стороны,

$$
\begin{gathered}
\mathscr{K}=\mathscr{K}_{0}+\Delta_{0} \mathscr{K}, \\
\left|\Delta_{0} \mathscr{K}\right| \leqslant C \Omega y_{0}^{3} \overline{S^{(3)}} .
\end{gathered}
$$

Определим величину $\Delta \mathscr{K}$ равенством

$$
\mathscr{K} \equiv \mathscr{K}\left[\rho e^{i \Omega S}\right]=i \pi \operatorname{sgn} b_{1}+\Delta \mathscr{K} .
$$

Лемма П.5. Пусть $\Omega\left|b_{2}\right| y_{0}^{2}>C \Omega^{\varepsilon^{\prime}}, \varepsilon^{\prime}>0$. Тогда для величинь $\Delta \mathscr{K}$ в (П.76) имеет место равенство

$$
\Delta \mathscr{K}=\Delta A_{0}+\Delta_{0} \mathscr{K}+\Delta A_{\rho}+\Delta_{T}\left(\varepsilon^{\prime}\right) .
$$

Величина $\Delta_{0} A$ в правой части этого равенства определена в (П.73) и при $x \equiv$ $\Omega b_{1}^{2} /\left(4\left|b_{2}\right|\right)>C \Omega^{\varepsilon^{\prime}}$ выполняется (П.74). Величина $\Delta_{0} \mathscr{K}$ оченена в (П.75), $\Delta_{T}\left(\varepsilon^{\prime}, \theta\right) \leqslant C_{T}\left(\varepsilon^{\prime}, \theta\right) \Omega^{-T} \quad(\forall T) u$

$$
\Delta A_{p}= \begin{cases}0 & \left(\left|b_{1}\right|<2\left|b_{2}\right| y_{0}\right), \\ \frac{1}{\sqrt{x}} e^{i \frac{\pi}{4} \operatorname{sgn} b_{2}-i x} \sum_{p=0}^{P_{T}\left(\varepsilon^{\prime}\right)} \frac{G_{p}\left(y_{c}\right)}{x^{p}} & \left(\left|b_{1}\right| \geqslant 2\left|b_{2}\right| y_{0}\right),\end{cases}
$$

где функиии $G_{p}(y)$ и величинь $y_{c}$ определяются из условий

$$
\left(\frac{\rho-1}{y}\right)^{(2 p)}(y)=y^{-2 p-1} G_{p}(y), \quad y_{c}=-\frac{b_{1}}{2 b_{2}} .
$$

Таким образом, во всей области изменения переменной $x=\frac{\Omega b_{1}^{2}}{4\left|b_{2}\right|}$

$$
\Delta \mathscr{K}=g(\sqrt{x}) e^{i x \operatorname{sgn} b_{1}}+\Delta_{0} \mathscr{K}+\Delta_{T}\left(\varepsilon^{\prime}, \theta\right),
$$

где $g$-ограниченная кусочно-гладкая функция. 
Лемма П.6. При $\Omega \geqslant 1$ равномерно по $b_{1}, b_{2}, \forall \varepsilon>0, \theta$ имеет место оценка

$$
|\Delta \mathscr{K}| \leqslant \frac{C}{\left|b_{1}\right|}\left[\frac{1}{\Omega^{1+\varepsilon} y_{0}}+\frac{\left|b_{2}\right|^{1 / 2}}{\Omega^{1 / 2}}+\frac{\left(\overline{S^{(3)}}\left|b_{1}\right|\right)^{1 / 4}}{\Omega^{1 / 2}}+\overline{S^{(3)}} y_{0}^{2}+\bar{S}^{(4)} y_{0}^{3}\right] .
$$

\section{Список обозначений}

В списке приведены номера формул, в окрестности которых вводятся соответствующие обозначения. В список включены только те обозначения, которые безпояснений используются в тексте и смысл которых может быть не ясен из контекста.

$a, a_{0}, a_{i}, a_{B}(5.4)-(5.5) ; A,\{A\}(1.7) ; A_{i}, i=1, \ldots, 6(2.2)-(2.5) ; A_{0}(2.6) ; A(n)(3.4)$; $A_{\Phi}(\Pi .2) ; A_{c}[u], A[u, \Phi](\Pi .20)$.

$b(2.7) ; B(3.25) ; B_{c}, B_{0}$ (П.4)-(П.5). $d \equiv d\left(\vec{r}_{0}, \partial \mathscr{D}\right)(1.29),(5.10) ; d_{0}(5.10) ; d_{T}(2.34) ;$ $D^{\prime}(2.25)-(2.26) ; D_{\beta_{1} \beta_{2}}^{i}, i=1,2,3(2.1) ; D_{0}(5.10) ; D_{i}(5.23)$.

$D_{q}^{c}(\Pi .6) ; \mathscr{D}, \partial \mathscr{D}(1.1)$.

$E(\vec{z})(3.5)$.

$\widehat{f}(1.2) ; \widetilde{f}, f_{0}(2.15)$.

$g_{1}, \widetilde{g}(\Pi .30)-(\Pi .31) ; G_{i}\left(A, \vec{r}_{0}\right), G_{0}\left(A, \vec{r}_{0}\right)(2.24)$.

$h_{n}^{\mathrm{sym}}, h_{n}^{\text {ant }}(\Pi .57)-(\Pi .58) ; H^{A}(s)(1.9)-(1.11)$.

$j_{0}, j_{1}(2.22) ; j_{i n}^{0}(2.20) ; j_{i n}^{1}(2.21) ; J(\varphi, q)(1.2) ; J^{\Pi}(\varphi, q)(1.4) ; J_{n m}(1.33) ; J_{\partial M}^{P}$ (П.3).

$k(s)$ (1.36), (2.7)-(2.10); $\bar{k}(2.7) ; \widetilde{k}(2.9) ; k_{d}(5.26) ; k_{D}(5.25) ; K_{i}, K_{0}(2.7) ; \widetilde{K}_{p}(2.34)$;

$\mathscr{K}_{ \pm}(5.47) ; \mathscr{K}_{c 1}(\Pi .45) ; \mathscr{K}\left[f e^{i \Omega S}\right](\Pi .43)$.

$\vec{l}(s)(1.34),(4.1) ; l \equiv|\vec{l}(s)|, L, L_{0}(1.6), \mathscr{L}(2.10)$.

$M(3.16)-(3.27)$.

$n_{0}, \bar{n}_{0}(\Pi .39) ; N_{\varphi}(1.3) ; N_{1}(3.25) ; \mathscr{N}_{1}(5.23)$.

$\vec{O}(1.1)$.

$p, p_{i}(2.8)$.

$q(2.12)$.

$\vec{r}_{0}, \vec{r}(s)(1.34) ; \vec{r}_{m}^{0}(2.10) ; \vec{r}_{0 i}(2.25)-(2.26) ; \vec{r}_{\Gamma}(4.20) ; R_{H}(1.1) ; R_{0}(1.6) ; R^{A}(1.11) ;$ $R(2.28) ; R_{1}(2.32)$.

$s$ (1.34); $s_{c}^{1}(2.25) ; s_{c}^{i}(4.8) ; s_{0 c}^{1}(2.25)-(2.26),(5.32) ; s_{i}^{0}$ (2.1), (2.8); $S^{A}$ (1.13), (2.3); $S_{\Pi}(1.13),(2.2) ; S_{R}^{A}(2.3) ; S_{ \pm}(s), S(s)(4.5) ; S_{\gamma}, S_{\gamma}^{0}(4.16)-(4.17)$.

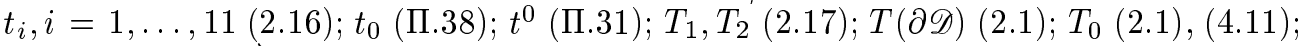
$T\left(s_{i}^{0}\right)(2.1) ; T(\vec{O})(4.20)-(4.21) ; T_{D_{i}}(5.23), T_{j}(\Omega)(\Pi .57)-(\Pi .58) ; T_{\text {sym }}, T_{\text {ant }}(\Pi .60) ;$ $T_{\text {sym(ant) }}^{q}(\Pi .61)$.

$u_{n}(1.35) ; U(\beta, M), U\left(\beta_{1}, \partial \mathscr{D}\right), U\left(\beta_{2}, T_{0}\right)(2.1) ; U\left(\beta_{2}, T\left(s_{i}^{0}\right)\right), U\left(\beta_{3}, r_{m}^{0}\right)(2.25)$.

$v_{n}(1.35) ; V^{\Delta t}, V_{\Delta t}(1.6)-(1.7)$.

$w_{n m}^{ \pm}(5.15)-(5.16) ; w_{n m}^{ \pm i}, i=1,2,3(5.47)-(5.48) ; w_{j}(\varphi, s)(5.75)$.

$x_{c}, x_{\mathrm{ex}}(\Pi .4)-(\Pi .5)$.

$\alpha_{i}, i=1, \ldots, 11, \alpha(2.26)$.

$\beta_{1}, \beta_{2}(2.1),(5.10) ; \beta_{3}, \ldots, \beta_{6}(5.10)$.

$\gamma(5.90) ; \Gamma_{\gamma}, \Gamma_{0}(4.20)$.

$\Delta \varphi, \Delta q(1.3) ; \Delta \mu^{A}(1.22) ; \Delta \mu_{R}^{A}(1.26) ; \Delta \mu_{T}^{A}(1.28) ; \Delta \mu_{q}^{A}(2.7) ; \Delta \mu_{\varphi}^{A}, \Delta \mu_{L}^{A}(3.2)-(3.3) ;$

$\Delta \mu_{T F}^{A}(3.25) ; \Delta \mu_{F}^{A}(3.26) ; \Delta \xi(2.4) ; \Delta^{n_{0}} J_{2}$ (П.48); $\Delta_{i}(2.9) ; \Delta^{0}(2.14)$.

$\varepsilon, \varepsilon_{0}(2.26) ; \varepsilon_{1}, \varepsilon_{2}$ (Теоремы П.1-П.5). 


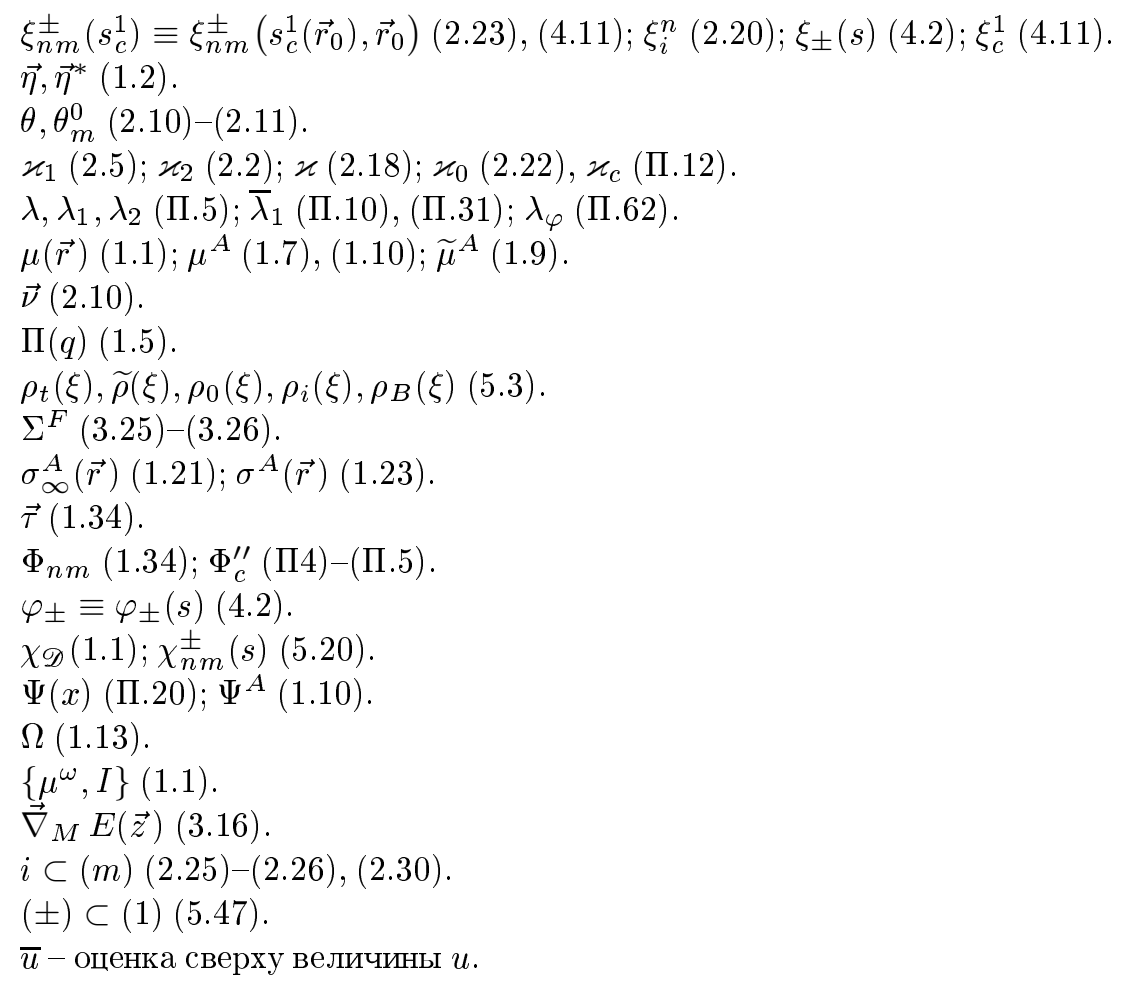

\section{СПИСОК ЛИТЕРАТУРЫ}

[1] Хермен Г. Восстановление изображения по проекциям: Основы реконструктивной томографии. М.: Мир, 1983.

[2] Пикалов В.В., Преображенский Н.Г. Реконструктивная томография в газодинамике и физике плазмы. Новосибирск: Наука, 1987.

[3] Реконструктивная вычислительная томограффия // ТИИЭР. 1983. Т. 71. № 3.

[4] Herman G.T. (Ed.) Image Reconstruction from Projections: Implementation and Application // Topics in Appl. Phys. 1979. V. 39. N. Y.

[5] Наттерер Ф. Математические аспекты компьютерной томографии. М.: Мир, 1990.

[6] Herman G.T., Natterer F. (Eds.) Mathematical Aspects of Computerized Tomography // Lecture Notes in Med. Inform. V. 8. New York: Springer-Verlag, 1981.

[7] Shepp L. A. (Ed.) Computed Tomography. Providence, RI: Amer. Math. Soc., 1983 (Proc. Sympos. Appl. Math. V. 27).

[8] Smith K.T., Solmon D. C., Wagner S. L. Practical and mathematical aspects of the problem of reconstructing objects from radiographs // Bull. Amer. Math. Soc. 1977. V. 83. №6. P. 1127-1270.

[9] Shepp L. A., Kruskal J. B. Computerized tomography: the new medical $X$-ray technology // Amer. Math. Monthly. 1978. V. 85. P. 420-439.

[10] Gelfand I. M., Gindikin S. G. (Eds.) Mathematical Problems of Tomography. Providence, RI: Amer. Math. Soc., 1990 (Transl. Math. Monographs. V. 81).

[11] Gindikin S. (Ed.) Applied Problems of Radon Transform. Providence, RI: Amer. Math. Soc., 1994.

[12] Popov D. A., Sokolova E. B., Sushko D. V. Mathematical models in two-dimensional Radon tomography // [11]. P. 129-204.

[13] Palamodov V.P. Some singular problems in tomography // [10]. P. 123-140.

[14] Рамм А. Г. Многомерные обратные задачи рассеяния. М.: Мир, 1994. 
[15] Гельфанд И. М., Граев М. И., Виленкин Н. Я. Интегральная геометрия и связанные с ней вопросы теории представлений. Обобщенные функции. Вып. 5. М.: Физматгиз, 1962.

[16] Хелгасон С. Преобразование Радона. М.: Мир, 1983.

[17] Гельфанд И. М., Шилов Г. Е. Обобщенные функции и действия над ними. Обобщенные функции. Вып. 1. М.: Физматгиз, 1959.

[18] Popov D. A., Sushko D. V. Computation of singular convolutions // [11]. P. 43-127.

[19] Popov D. A. On convergence of a class of algorithms for the inversion the numerical Radon transform // [10]. P. 7-65.

[20] Гончар С. И. Приближение функций при помощи дискретного обращения их преобразования Радона // Дис. ... канд. физ.-матем. наук. М.: МГУ, 1987.

[21] Гончар С. И. Сходимость вычислительных алгоритмов восстановления разрьвных функций по преобразованию Радона // УМН. 1986. Т. 41. № 3. С. 175-176.

[22] Попов Д.А. Исследование алгоритма свертки и обратной проекции // Вопросы реконструктивной томографии. Новосибирск: ВЦ СО АН СССР, 1985. С. 125-136.

[23] Арнольд В.И., Варченко А. Н., Гусейн-Заде С. М. Особенности дифференцируемых отображений. Т. 1. М.: Наука, 1982.

[24] Брус Дж., Джиблин П. Кривые и особенности. М.: Мир, 1988.

[25] Попов Д. А. Оценки с константами для некоторого класса осциллирующих интегралов // УМН. 1997. Т. 52. № 1. С. 77-148.

[26] Погорелов А. В. Лекции по дифференциальной геометрии. Харьков: Изд-во Харьковского ун-та, 1967.

[27] Алимов И.А., Ильин В. А., Никишин Е. Е. Вопросы сходимости кратных тригонометрических рядов и спектральных разложений. I // УМН. 1976. Т. 31. №6. С. 28-83.

[28] Янушкаускас А. И. Кратные тригонометрические ряды. Новосибирск: Наука, 1986.

[29] Ахиезер Н. И. Лекции по теории аппроксимации. М.: Наука, 1965.

[30] Ватсон Г. Н. Теория Бесселевых функций. Ч. І. М.: ИЛ, 1949.

[31] Федорюк М. В. Метод перевала. М.: Наука, 1977.

[32] Архипов Г. И., Карацуба А. А., Чубариков В.Н. Тригонометрические интегралы // Изв. АН СССР. Сер. матем. 1979. Т. 43. № 5. С. 971-1003.

[33] Архипов Г.И., Карацуба А. А., Чубариков В.Н. Теория кратных тригонометрических сумм. М.: Наука, 1987.

Московский государственньй

Поступила в редакцию

университет им. М. В. Ломоносова

06.01.1997 